\title{
Scattering-matrix approach to Casimir-Lifshitz force and heat transfer out of thermal equilibrium between arbitrary bodies
}

\author{
Riccardo Messina ${ }^{1}$ and Mauro Antezza ${ }^{2,3}$ \\ ${ }^{1}$ LNE-SYRTE, Observatoire de Paris, CNRS UMR 8630, UPMC, 61 avenue de l'Observatoire, F-75014 Paris, France \\ ${ }^{2}$ Université Montpellier 2, Laboratoire Charles Coulomb UMR 5221, F-34095, Montpellier, France \\ ${ }^{3}$ CNRS, Laboratoire Charles Coulomb UMR 5221, F-34095, Montpellier, France
}

(Received 9 July 2011; published 3 October 2011)

\begin{abstract}
We study the radiative heat transfer and the Casimir-Lifshitz force occurring between two bodies in a system out of thermal equilibrium. We consider bodies of arbitrary shape and dielectric properties, held at two different temperatures and immersed in environmental radiation at a third different temperature. We derive explicit closed-form analytic expressions for the correlations of the electromagnetic field and for the heat transfer and Casimir-Lifshitz force in terms of the bodies' scattering matrices. We then consider some particular cases which we investigate in detail: the atom-surface and the slab-slab configurations.
\end{abstract}

DOI: 10.1103/PhysRevA.84.042102

PACS number(s): $12.20 .-\mathrm{m}, 42.50 . \mathrm{Ct}, 44.40 .+\mathrm{a}$

\section{INTRODUCTION}

The interaction of an electromagnetic field with bodies gives rise to several effects, among which are the radiative heat transfer [1] and the Casimir-Lifshitz force [2-4]. These two effects are intimately connected and can be described by a common formalism. If a definite temperature is assigned to the bodies and radiation, two main situations are possible. The radiation can be at thermal equilibrium with the matter everywhere: in this case the heat transfer is identically zero, whereas the Casimir-Lifshitz force assumes its equilibrium value. If, instead the radiation is not at thermal equilibrium with the matter, both the appearance of heat transfer between the bodies and a variation of the forces acting on them occur.

In considering the phenomena of heat transfer and CasimirLifshitz forces, the bodies' shapes, geometric configurations, and dielectric functions are main issues, together with the thermal configuration of the system. A general framework used in solving such problems is that of macroscopic electrodynamics [4-6]. The Casimir-Lifshitz interaction for systems at thermal equilibrium (at $T \geqslant 0$ ) has been much studied in the last 60 years, a consistent and almost complete theoretical formulation has been reached, and it has been experimentalally observed [7]. In particular, much more recently, the thermal component of the force at thermal equilibrium has been measured [8]. In contrast, systems out of thermal equilibrium have been much less explored, and mainly simple configurations and idealized cases (such as infinite bodies) have been considered. Nonetheless, systems out of thermal equilibrium have shown remarkable features, already the object of theoretical and experimental investigations concerning both the force [9-19] and the heat transfer [20-27]. This is the case of the atomsurface force, where the absence of particular cancellations among the different components of the radiation out of thermal equilibrium leads to large quantitative and qualitative modifications such as new asymptotic behaviors and the possibility of repulsive interactions [9]. These new features have allowed the first experimental observation of thermal effects [12]. Recently, motivated by the necessity of developing a more complete theory for systems out of thermal equilibrium, several studies have been developed [28-33]. In particular, a general closed-form analytic expression for both the heat transfer and Casimir-Lifshitz interactions has been derived, valid for arbitrary body shapes and dielectric functions, and arbitrary temperatures of the bodies and of the environment [28]. For this purpose a scattering-matrix approach has been used. This approach has already been successfully employed to calculate Casimir-Lifshitz interactions [16,28,34,35]: its main advantage with respect to the standard Green-function formulation, where the electromagnetic problem needs to be solved for the complete system, consists in requiring an independent solution for each body composing the system.

In this paper we provide a systematic derivation for the heat transfer and for the Casimir-Lifshitz force out of thermal equilibrium between two bodies at two different temperatures, immersed in external environmental radiation characterized by another temperature [28]. Particular attention is devoted to the expression for the average values of the electromagnetic field in terms of the scattering operators. The general expression is finally analytically and numerically applied to several simple but already interesting configurations, by calculating the heat transfer and the force for slab-slab and atom-slab systems in an out-of-equilibrium scenario. The roles of finite-size effects and of the environmental temperature are shown to be qualitatively and quantitatively relevant.

The organization of the paper is the following. In Sec. II we present the physical system, the hypotheses, and the general theoretical framework. In Secs. III and IV we evaluate the analytical expression and the flux of the two main ingredients of our calculation: the Maxwell stress tensor and the Poynting vector. Section $\mathrm{V}$ is dedicated to the definition of the reflection and transmission operators associated with each body. Then, in Sec. VI the correlators of the fields emitted by the bodies and by the environment are expressed as a function of the scattering operators. This allows us to calculate, in Sec. VII, the fluxes of the stress tensor and the Poynting vector in any region and then the final expressions of the Casimir-Lifshitz force and the heat transfer. This expression is first applied to the case of a single body alone out of thermal equilibrium in Sec. VIII. Then, in Sec. IX, we discuss the case of the force 
acting on a neutral atom in front of a planar slab, as well as both the force and the heat transfer in a two-slab configuration. We finally give some concluding remarks.

\section{PHYSICAL SYSTEM AND ELECTROMAGNETIC FIELD}

Let us start by describing the geometrical configuration of our physical system. We are going to deal with two bodies, labeled with 1 and 2 . From a geometrical point of view we will assume that the two bodies are separated by a planar surface. This hypothesis is not strictly necessary in a scattering-matrix approach but it is nonetheless verified in all the relevant experimental configurations such as, for example, two parallel planes, a sphere or a cylinder in front of a plane, two cylinders, and an atom in the proximity of a planar surface. At the same time, this assumption allows us to choose a convenient plane-wave decomposition for the electric and magnetic fields, leading in this way to quite simple expressions for the Casimir-Lifshitz force and the heat transfer. To be more specific, the geometry of our system is depicted in Fig. 1: the bodies 1 and 2 are respectively enclosed in the strips $z_{1}<z<z_{2}$ and $z_{3}<z<z_{4}$, where $z_{2}<z_{3}$. As a consequence, any plane $z=\bar{z}$ with $z_{2}<\bar{z}<z_{3}$ separates the two bodies, and three regions $\mathrm{A}, \mathrm{B}$, and $\mathrm{C}$ are defined. Our geometrical description coherently includes as a limiting case the possibility of bodies having infinite thickness, taking for example for body 1 (body 2 ) the limit $z_{1} \rightarrow-\infty\left(z_{4} \rightarrow+\infty\right)$.

As far as the thermodynamical description of our system is concerned, we will assume that we are able to define for body 1 (2) a temperature $T_{1}\left(T_{2}\right)$ and that it is in local thermal equilibrium, i.e., the temperature of each body is assumed to be constant. Moreover, we assume that the two bodies are immersed in a vacuum environment (having $\varepsilon=1$ ) characterized by a third temperature $T_{3}$. We will make the further important assumption that the composite system is in a stationary regime, which means that the three temperatures remain constant in time.

Let us now first describe the general framework of the calculation. We are interested in calculating the force $\mathbf{F}$ acting on either of the two bodies, as well as the heat transfer $H$ into it, defined as the energy it absorbs per unit of time. If we focus for example on body 1 , these two quantities can be expressed

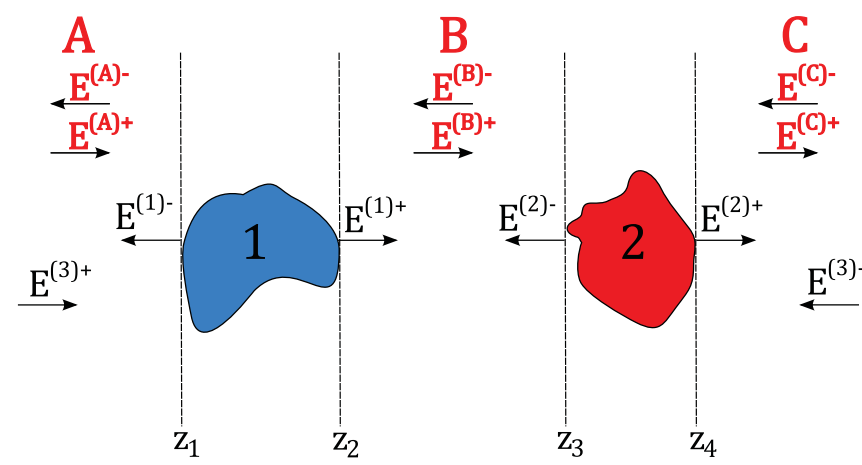

FIG. 1. (Color online) The geometry of the system. The bodies are separated by the strip $z_{2} \leqslant z \leqslant z_{3}$. This defines the three regions $\mathrm{A}, \mathrm{B}$, and $\mathrm{C}$. in the form of surface integrals through a closed surface $\Sigma$ enclosing the body 1 ,

$$
\begin{array}{r}
\mathbf{F}=\int_{\Sigma}\langle\mathbb{T}(\mathbf{R}, t)\rangle_{\mathrm{sym}} \cdot d \mathbf{\Sigma}, \\
H=-\int_{\Sigma}\langle\mathbf{S}(\mathbf{R}, t)\rangle_{\mathrm{sym}} \cdot d \mathbf{\Sigma},
\end{array}
$$

of the quantum symmetrized average of the Maxwell stress tensor $\mathbb{T}$ (having Cartesian components $T_{i j}$, with $i, j=x, y, z$ ) and the Poynting vector $\mathbf{S}$. These quantities are classically defined in SI units as

$$
\begin{aligned}
T_{i j}(\mathbf{R}, t)= & \epsilon_{0}\left\{E_{i}(\mathbf{R}, t) E_{j}(\mathbf{R}, t)+c^{2} B_{i}(\mathbf{R}, t) B_{j}(\mathbf{R}, t)\right. \\
& \left.-\frac{1}{2}\left[E^{2}(\mathbf{R}, t)+c^{2} B^{2}(\mathbf{R}, t)\right] \delta_{i j}\right\}, \\
\mathbf{S}(\mathbf{R}, t)= & \epsilon_{0} c^{2} \mathbf{E}(\mathbf{R}, t) \times \mathbf{B}(\mathbf{R}, t),
\end{aligned}
$$

and the quantum symmetrized average value $\langle A B\rangle_{\mathrm{sym}}$ is defined as

$$
\langle A B\rangle_{\mathrm{sym}}=\frac{1}{2}(\langle A B\rangle+\langle B A\rangle),
$$

where $\langle A\rangle$ is an ordinary quantum average value. Before working on Eq. (1) it is evident that a complete description of the electric and magnetic fields in any region is mandatory.

In our coordinate system the $z$ axis clearly represents a privileged direction, since it is the axis perpendicular to the plane separating the two bodies. Inspired by this property, for the mode decomposition of our electromagnetic field in any region we replace the common plane-wave representation in which a mode of the field is represented by the threedimensional wave vector $\mathbf{K}=\left(k_{x}, k_{y}, k_{z}\right)$ by a description in terms of the transverse wave vector $\mathbf{k}=\left(k_{x}, k_{y}\right)$ and the frequency $\omega$. The result of this choice is that in this approach the $z$ component $k_{z}$ of the wave vector becomes a dependent variable, defined by the relation $k_{z}^{2}=\frac{\omega^{2}}{c^{2}}-\mathbf{k}^{2}$. Since this relation is quadratic in $k_{z}$, we are obliged to introduce explicitly a variable $\phi$ taking the values $\phi= \pm 1$ (with shorthand notation $\phi= \pm$ in the expressions for the polarization vectors and field amplitudes) corresponding to the sign of $k_{z}$. As a consequence, the complete wave vector will be denoted as

$$
\begin{gathered}
\mathbf{K}^{\phi}=\left(\mathbf{k}, \phi k_{z}\right)=\left(k_{x}, k_{y}, \phi k_{z}\right), \\
k_{z}=\sqrt{\frac{\omega^{2}}{c^{2}}-\mathbf{k}^{2}} .
\end{gathered}
$$

For $k \leqslant \frac{\omega}{c}$, this relation gives a real value of $k_{z}$, and then a propagative wave, for which $\phi$ represents the direction of propagation along the $z$ axis. In contrast, for $k>\frac{\omega}{c}, k_{z}$ becomes imaginary, and we get an evanescent wave whose amplitude depends on $z$ : in this case $\phi$ is the direction along which the amplitude of the evanescent wave decays. Moreover, we will as usual need an index $p$ associated with the polarization, taking the values $p=1,2$ corresponding to TE and TM modes, respectively. Finally, in our approach a mode of the field is identified by the set of variables $(\omega, \mathbf{k}, p, \phi)$.

The expression for the electric field in any region can be first given in the form of a frequency decomposition

$$
\mathbf{E}(\mathbf{R}, t)=2 \operatorname{Re}\left[\int_{0}^{+\infty} \frac{d \omega}{2 \pi} \exp (-i \omega t) \mathbf{E}(\mathbf{R}, \omega)\right],
$$


where a single-frequency component has the following mode decomposition:

$$
\mathbf{E}(\mathbf{R}, \omega)=\sum_{\phi, p} \int \frac{d^{2} \mathbf{k}}{(2 \pi)^{2}} \exp \left(i \mathbf{K}^{\phi} \cdot \mathbf{R}\right) \hat{\boldsymbol{\epsilon}}_{p}^{\phi}(\mathbf{k}, \omega) E_{p}^{\phi}(\mathbf{k}, \omega) .
$$

From now on the sum on $\phi$ runs over the values $\{+,-\}$ and the sum on $p$ over the values $\{1,2\}$. The quantity $E_{p}^{\phi}(\mathbf{k}, \omega)$ represents the amplitude of the electric field associated with a given mode $(\omega, \mathbf{k}, p, \phi)$. For the polarization vectors $\hat{\boldsymbol{\epsilon}}_{p}^{\phi}(\mathbf{k}, \omega)$ appearing in Eq. (6) we adopt the following standard definitions:

$$
\begin{gathered}
\hat{\boldsymbol{\epsilon}}_{\mathrm{TE}}^{\phi}(\mathbf{k}, \omega)=\hat{\mathbf{z}} \times \hat{\mathbf{k}}=\frac{1}{k}\left(-k_{y} \hat{\mathbf{x}}+k_{x} \hat{\mathbf{y}}\right), \\
\hat{\boldsymbol{\epsilon}}_{\mathrm{TM}}^{\phi}(\mathbf{k}, \omega)=\frac{c}{\omega} \hat{\boldsymbol{\epsilon}}_{\mathrm{TE}}^{\phi}(\mathbf{k}, \omega) \times \mathbf{K}^{\phi}=\frac{c}{\omega}\left(-k \hat{\mathbf{z}}+\phi k_{z} \hat{\mathbf{k}}\right),
\end{gathered}
$$

where $\hat{\mathbf{x}}, \hat{\mathbf{y}}$, and $\hat{\mathbf{z}}$ are the unit vectors along the directions $x$, $y$, and $z$ respectively and $\hat{\mathbf{k}}=\mathbf{k} / k$. The unit vectors defined in (7) have the following useful properties:

$$
\begin{gathered}
\hat{\boldsymbol{\epsilon}}_{\mathrm{TE}}^{\phi}(-\mathbf{k}, \omega)=-\hat{\boldsymbol{\epsilon}}_{\mathrm{TE}}^{\phi}(\mathbf{k}, \omega), \quad \hat{\boldsymbol{\epsilon}}_{\mathrm{TE}}^{-\phi}(\mathbf{k}, \omega)=\hat{\boldsymbol{\epsilon}}_{\mathrm{TE}}^{\phi}(\mathbf{k}, \omega), \\
{\left[\hat{\boldsymbol{\epsilon}}_{\mathrm{TE}}^{\phi}(\mathbf{k}, \omega)\right]^{*}=\hat{\boldsymbol{\epsilon}}_{\mathrm{TE}}^{\phi}(\mathbf{k}, \omega), \quad \hat{\boldsymbol{\epsilon}}_{\mathrm{TM}}^{\phi}(-\mathbf{k}, \omega)=\hat{\boldsymbol{\epsilon}}_{\mathrm{TM}}^{-\phi}(\mathbf{k}, \omega),} \\
{\left[\hat{\boldsymbol{\epsilon}}_{\mathrm{TM}}^{\phi}(\mathbf{k}, \omega)\right]^{*}= \begin{cases}\hat{\boldsymbol{\epsilon}}_{\mathrm{TM}}^{\phi}(\mathbf{k}, \omega), & k_{z} \in \mathbb{R}, \\
\hat{\boldsymbol{\epsilon}}_{\mathrm{TM}}^{-\phi}(\mathbf{k}, \omega), & k_{z} \notin \mathbb{R} .\end{cases} }
\end{gathered}
$$

The expression for the magnetic field can be easily deduced from Maxwell's equations, and is given by

$$
\mathbf{B}(\mathbf{R}, \omega)=\frac{1}{c} \sum_{\phi, p} \int \frac{d^{2} \mathbf{k}}{(2 \pi)^{2}} \exp \left(i \mathbf{K}^{\phi} \cdot \mathbf{R}\right) \hat{\boldsymbol{\beta}}_{p}^{\phi}(\mathbf{k}, \omega) E_{p}^{\phi}(\mathbf{k}, \omega),
$$

where

$$
\hat{\boldsymbol{\beta}}_{p}^{\phi}(\mathbf{k}, \omega)=(-1)^{p} \hat{\boldsymbol{\epsilon}}_{S(p)}^{\phi}(\mathbf{k}, \omega) .
$$

$S(p)$ is the function which switches between the two polarizations, acting as $S(1)=2$ and $S(2)=1$. We are going to gather the expressions (6) and (9) for the electric and magnetic fields at a given frequency $\omega$ in a column vector and write

$$
\begin{aligned}
\left(\begin{array}{c}
\mathbf{E}(\mathbf{R}, \omega) \\
c \mathbf{B}(\mathbf{R}, \omega)
\end{array}\right)= & \sum_{\phi, p} \int \frac{d^{2} \mathbf{k}}{(2 \pi)^{2}} \exp \left(i \mathbf{K}^{\phi} \cdot \mathbf{R}\right) \\
& \times \hat{\boldsymbol{\epsilon}}_{A(p)}^{\phi}(\mathbf{k}, \omega)\left(\begin{array}{c}
1 \\
(-1)^{p}
\end{array}\right) E_{p}^{\phi}(\mathbf{k}, \omega),
\end{aligned}
$$

where in the right-hand side the 1 and the $(-1)^{p}$ correspond to the electric and magnetic fields, respectively, and we have introduced the vectorial index

$$
A(p)=\left(\begin{array}{c}
p \\
S(p)
\end{array}\right)
$$

Here again the upper (lower) index is associated with the electric (magnetic) field.

Now that the expressions for the electric and magnetic fields are explicitly given in terms of a set of field amplitudes $E_{p}^{\phi}(\mathbf{k}, \omega)$, we are ready to work, in the next sections, on the explicit expressions for the Maxwell stress tensor and Poynting vector.

\section{THE MAXWELL STRESS TENSOR}

\section{A. General expression for the tensor}

From the definition of the stress tensor (2) it is clear that we need to calculate explicitly the quantities $E_{i} E_{j}$ and $B_{i} B_{j}$ for $i, j=x, y, z$ and as a further step their symmetrized quantum average. Using the compact vectorial notation introduced in Eq. (11), we have

$$
\begin{aligned}
\left(\begin{array}{c}
E_{i} E_{j} \\
c^{2} B_{i} B_{j}
\end{array}\right)= & \sum_{\phi \phi^{\prime}} \sum_{p p^{\prime}} \int \frac{d^{2} \mathbf{k}}{(2 \pi)^{2}} \int \frac{d^{2} \mathbf{k}^{\prime}}{(2 \pi)^{2}} \int_{0}^{+\infty} \frac{d \omega}{2 \pi} \\
& \times \int_{0}^{+\infty} \frac{d \omega^{\prime}}{2 \pi}\left(\begin{array}{c}
1 \\
(-1)^{p+p^{\prime}}
\end{array}\right) \exp \left[i\left(\mathbf{k}-\mathbf{k}^{\prime}\right) \cdot \mathbf{r}\right] \\
& \times \exp \left[i\left(\phi k_{z}-\phi^{\prime} k_{z}^{\prime *}\right) z\right] \exp \left[-i\left(\omega-\omega^{\prime}\right) t\right] \\
& \times\left\{E_{p}^{\phi}(\mathbf{k}, \omega) E_{p^{\prime}}^{\phi^{\prime} \dagger}\left(\mathbf{k}^{\prime}, \omega^{\prime}\right)\left[\hat{\epsilon}_{A(p)}^{\phi}(\mathbf{k}, \omega)\right]_{i}\right. \\
& \times\left[\hat{\epsilon}_{A\left(p^{\prime}\right)}^{\phi^{\prime} *}\left(\mathbf{k}^{\prime}, \omega^{\prime}\right)\right]_{j}+E_{p^{\prime}}^{\phi^{\dagger} \dagger}\left(\mathbf{k}^{\prime}, \omega^{\prime}\right) E_{p}^{\phi}(\mathbf{k}, \omega) \\
& \left.\times\left[\hat{\epsilon}_{A(p)}^{\phi}(\mathbf{k}, \omega)\right]_{j}\left[\hat{\epsilon}_{A\left(p^{\prime}\right)}^{\phi^{\prime} *}\left(\mathbf{k}^{\prime}, \omega^{\prime}\right)\right]_{i}\right\}+\mathcal{Z}
\end{aligned}
$$

where the fields implicitly depend on $(\mathbf{R}, t)$, and $\mathcal{Z}$ generically gathers all the terms proportional to $E E$ or $E^{\dagger} E^{\dagger}$ whose average quantum value is zero. Having in mind the transition to the quantum symmetrized average values, we now give the following definition for the commutators $C^{\phi \phi^{\prime}}$ of the field amplitudes:

$$
\begin{aligned}
\left\langle E_{p}^{\phi}(\mathbf{k}, \omega) E_{p^{\prime}}^{\phi^{\prime} \dagger}\left(\mathbf{k}^{\prime}, \omega^{\prime}\right)\right\rangle_{\mathrm{sym}}= & \frac{1}{2}\left\langle E_{p}^{\phi}(\mathbf{k}, \omega) E_{p^{\prime}}^{\phi^{\prime} \dagger}\left(\mathbf{k}^{\prime}, \omega^{\prime}\right)\right. \\
& \left.+E_{p^{\prime}}^{\phi^{\prime} \dagger}\left(\mathbf{k}^{\prime}, \omega^{\prime}\right) E_{p}^{\phi}(\mathbf{k}, \omega)\right\rangle \\
= & 2 \pi \delta\left(\omega-\omega^{\prime}\right)\left\langle p, \mathbf{k}\left|C^{\phi \phi^{\prime}}\right| p^{\prime}, \mathbf{k}^{\prime}\right\rangle
\end{aligned}
$$

where we stress the fact that in general two modes of the field propagating in opposite directions do not necessarily commute. Moreover, we have explicitly inserted the conservation of frequency: since in our system no dynamics is considered, the field amplitudes at different frequencies necessarily commute as a consequence of time invariance. By virtue of this conservation any correlation function of the electromagnetic field analogous to (14) is a function of a single frequency $\omega$ (and not of both $\omega$ and $\omega^{\prime}$ ), which will not be explicitly written from now on. Moreover, in Eq. (14) we have expressed the correlators as matrix elements of a matrix $C^{\phi \phi^{\prime}}$ : in our notation, the matrices are defined on the space $(p, \mathbf{k})$ with $p=1,2$ and $\mathbf{k} \in \mathbb{R}^{2}$, and thus the product of two matrices $A$ and $B$ is given by

$$
\begin{aligned}
\left\langle p, \mathbf{k}|\mathcal{A B}| p^{\prime}, \mathbf{k}^{\prime}\right\rangle= & \sum_{p^{\prime \prime}} \int \frac{d^{2} \mathbf{k}^{\prime \prime}}{(2 \pi)^{2}}\left\langle p, \mathbf{k}|\mathcal{A}| p^{\prime \prime}, \mathbf{k}^{\prime \prime}\right\rangle \\
& \times\left\langle p^{\prime \prime}, \mathbf{k}^{\prime \prime}|\mathcal{B}| p^{\prime}, \mathbf{k}^{\prime}\right\rangle
\end{aligned}
$$

where the matrices $\mathcal{A}$ and $\mathcal{B}$ are both calculated at the same fixed frequency $\omega$. 
Using Eq. (13) and the definition (14) we have

$$
\begin{aligned}
\left\langle\left(\begin{array}{c}
E_{i} E_{j} \\
c^{2} B_{i} B_{j}
\end{array}\right)\right\rangle_{\mathrm{sym}}= & \sum_{\phi \phi^{\prime}} \sum_{p p^{\prime}} \int \frac{d^{2} \mathbf{k}}{(2 \pi)^{2}} \int \frac{d^{2} \mathbf{k}^{\prime}}{(2 \pi)^{2}} \int_{0}^{+\infty} \frac{d \omega}{2 \pi} \\
& \times\left(\begin{array}{c}
1 \\
(-1)^{p+p^{\prime}}
\end{array}\right) \exp \left[i\left(\mathbf{k}-\mathbf{k}^{\prime}\right) \cdot \mathbf{r}\right] \\
& \times \exp \left[i\left(\phi k_{z}-\phi^{\prime} k_{z}^{*}\right) z\right]\left\langle p, \mathbf{k}\left|C^{\phi \phi^{\prime}}\right| p^{\prime}, \mathbf{k}^{\prime}\right\rangle \\
& \times\left\{\left[\hat{\epsilon}_{A(p)}^{\phi}(\mathbf{k}, \omega)\right]_{i}\left[\hat{\epsilon}_{A\left(p^{\prime}\right)}^{\phi^{\prime} *}\left(\mathbf{k}^{\prime}, \omega\right)\right]_{j}\right. \\
& \left.+\left[\hat{\epsilon}_{A(p)}^{\phi}(\mathbf{k}, \omega)\right]_{j}\left[\hat{\epsilon}_{A\left(p^{\prime}\right)}^{\phi^{\prime}}\left(\mathbf{k}^{\prime}, \omega\right)\right]_{i}\right\}
\end{aligned}
$$

It is important to note that the correlator $C^{\phi \phi^{\prime}}$ defined in (14) and appearing in (16) depends on the region (A, B, or $\mathrm{C}$ ) in which the average is calculated: this information is contained in the $z$ dependence of the fields in the left-hand side of Eq. (16), as well as in the $z$ coordinate explicitly present in its right-hand side. As we will see in Sec. III B, we only need to evaluate the symmetrized average value of the flux of the $i z$ components $(i=x, y, z)$ of the electromagnetic stress tensor. These quantities can be calculated using Eqs. (2) and (16). In the case of $T_{z z}$ we have

$$
\begin{aligned}
\left\langle T_{z z}\right\rangle_{\mathrm{sym}}= & \epsilon_{0} \sum_{\phi \phi^{\prime}} \sum_{p p^{\prime}} \int \frac{d^{2} \mathbf{k}}{(2 \pi)^{2}} \int \frac{d^{2} \mathbf{k}^{\prime}}{(2 \pi)^{2}} \int_{0}^{+\infty} \frac{d \omega}{2 \pi} \\
& \times \exp \left[i\left(\mathbf{k}-\mathbf{k}^{\prime}\right) \cdot \mathbf{r}\right] \exp \left[i\left(\phi k_{z}-\phi^{\prime} k_{z}^{\prime *}\right) z\right] \\
& \times\left\langle p, \mathbf{k}\left|C^{\phi \phi^{\prime}}\right| p^{\prime}, \mathbf{k}^{\prime}\right\rangle\left\{\left[\hat{\epsilon}_{p}^{\phi}(\mathbf{k}, \omega)\right]_{z}\left[\hat{\epsilon}_{p^{\prime}}^{\phi^{\prime} *}\left(\mathbf{k}^{\prime}, \omega\right)\right]_{z}\right. \\
& -\left[\hat{\epsilon}_{p}^{\phi}(\mathbf{k}, \omega)\right]_{x}\left[\hat{\epsilon}_{p^{\prime}}^{\phi^{\prime} *}\left(\mathbf{k}^{\prime}, \omega\right)\right]_{x} \\
& -\left[\hat{\epsilon}_{p}^{\phi}(\mathbf{k}, \omega)\right]_{y}\left[\hat{\epsilon}_{p^{\prime}}^{\phi^{\prime} *}\left(\mathbf{k}^{\prime}, \omega\right)\right]_{y} \\
& +(-1)^{p+p^{\prime}}\left[\left[\hat{\epsilon}_{S(p)}^{\phi}(\mathbf{k}, \omega)\right]_{z}\left[\hat{\epsilon}_{S\left(p^{\prime}\right)}^{\phi^{\prime} *}\left(\mathbf{k}^{\prime}, \omega\right)\right]_{z}\right. \\
& -\left[\hat{\epsilon}_{S(p)}^{\phi}(\mathbf{k}, \omega)\right]_{x}\left[\hat{\epsilon}_{S\left(p^{\prime}\right)}^{\phi^{\prime} *}\left(\mathbf{k}^{\prime}, \omega\right)\right]_{x} \\
& \left.\left.-\left[\hat{\epsilon}_{S(p)}^{\phi}(\mathbf{k}, \omega)\right]_{y}\left[\hat{\epsilon}_{S\left(p^{\prime}\right)}^{\phi^{\prime} *}\left(\mathbf{k}^{\prime}, \omega\right)\right]_{y}\right]\right\}
\end{aligned}
$$

whereas for $T_{m z}(m=x, y)$ a straightforward calculation gives

$$
\begin{aligned}
\left\langle T_{m z}\right\rangle_{\mathrm{sym}}= & \epsilon_{0} \sum_{\phi \phi^{\prime}} \sum_{p p^{\prime}} \int \frac{d^{2} \mathbf{k}}{(2 \pi)^{2}} \int \frac{d^{2} \mathbf{k}^{\prime}}{(2 \pi)^{2}} \int_{0}^{+\infty} \frac{d \omega}{2 \pi} \\
& \times \exp \left[i\left(\mathbf{k}-\mathbf{k}^{\prime}\right) \cdot \mathbf{r}\right] \exp \left[i\left(\phi k_{z}-\phi^{\prime} k_{z}^{\prime *}\right) z\right] \\
& \times\left\langle p, \mathbf{k}\left|C^{\phi \phi^{\prime}}\right| p^{\prime}, \mathbf{k}^{\prime}\right\rangle\left\{\left[\left[\hat{\epsilon}_{p}^{\phi}(\mathbf{k}, \omega)\right]_{m}\left[\hat{\epsilon}_{p^{\prime}}^{\phi^{\prime}}\left(\mathbf{k}^{\prime}, \omega\right)\right]_{z}\right.\right. \\
& \left.+(-1)^{p+p^{\prime}}\left[\hat{\epsilon}_{S(p)}^{\phi}(\mathbf{k}, \omega)\right]_{m}\left[\hat{\epsilon}_{S\left(p^{\prime}\right)}^{\phi^{\prime} *}\left(\mathbf{k}^{\prime}, \omega\right)\right]_{z}\right] \\
& +[m \rightleftarrows z]\},
\end{aligned}
$$

where the symbol $m \rightleftarrows z$ represents two more terms obtained by interchanging in the first two $m$ with $z$. Let us make some comments on these expressions. First we note that, even if the stress tensor is in general a function of time, this is not true for its quantum average value. In addition, this is a function of the correlators of the electric-field amplitudes (propagating either in the same or in opposite directions) and of the components of the polarization unit vectors. As we will see in the next section, the components (17) and (18) are the only ones we need to calculate all the components of the force acting on the two bodies.

\section{B. Flux of the stress tensor in terms of field correlators}

As anticipated, the electromagnetic force acting on a given body inside a volume region can be calculated by taking the flux of the stress tensor through a closed surface enclosing this volume. As a consequence, the $m$ component of the force on a given body is given by the flux

$$
F_{m}=\int_{\Sigma}\left\langle T_{m j}\right\rangle_{\mathrm{sym}} d \Sigma_{j}
$$

where summation over repeated indices is assumed and $\Sigma$ represents any closed box entirely enclosing the body. Let us choose for example the box depicted in Fig. 2, i.e., a parallelepiped having one side of length $D$ and as a base orthogonal to the $z$ axis a square of side $L$. According to the definition of $T_{i j}$, the $m$ component of the force $(m=x, y, z)$ is given in this case by the flux of $T_{m z}$ through the two surfaces orthogonal to the $z$ axis, plus the fluxes of $T_{m x}$ and $T_{m y}$ through the surfaces of the parallelepiped orthogonal to the $x$ and $y$ axes, respectively. Taking now the limit $L \rightarrow+\infty$, we see that the surface of the two bases orthogonal to the $z$ axis diverges more rapidly (as $L^{2}$ ) than the other four surfaces (as $L)$. As a consequence, we deduce that in order to calculate $F_{m}$ one simply needs to calculate the flux of $T_{m z}$ on the surface (which has now become a plane) in region $\mathrm{A}$ and subtract this result from the flux of $T_{m z}$ through the plane in region B. Moreover, due to the arbitrariness of the box, these two fluxes must not depend on the $z$ coordinates of the respective planes, even if in general the stress tensor depends on $z$.

From this discussion we conclude that we need the flux of $T_{m z}$ through a plane $z=\bar{z}$. We will then have $\bar{z}<z_{1}$ for region $\mathrm{A}, z_{2}<\bar{z}<z_{3}$ for region $\mathrm{B}$, and $\bar{z}>z_{4}$ for region $\mathrm{C}$. Integrating Eq. (17) on the plane $z=\bar{z}$ and noticing that this

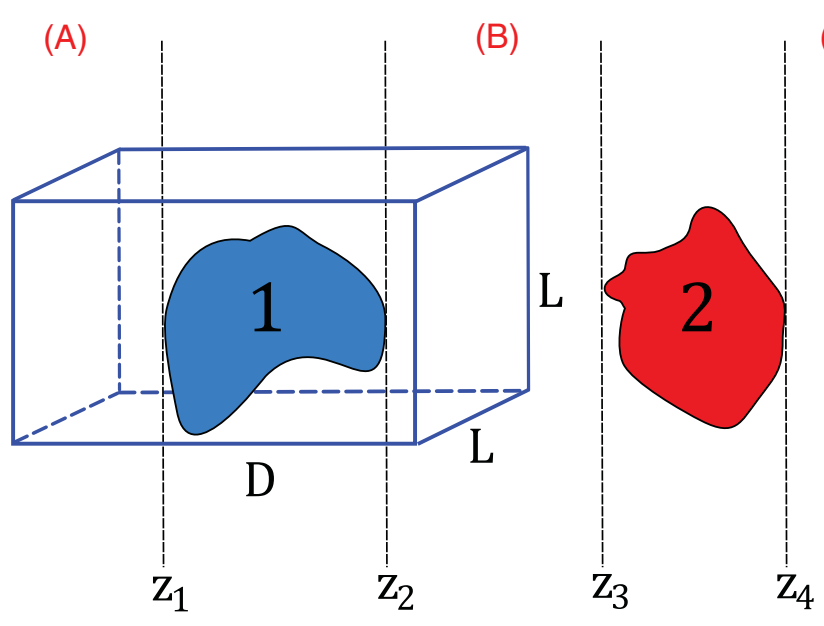

FIG. 2. (Color online) The box chosen for the calculation of the force acting on body 1 . 
gives a Dirac delta $(2 \pi)^{2} \delta\left(\mathbf{k}-\mathbf{k}^{\prime}\right)$, we get the flux of $T_{z z}$ expressed as a function of the field correlators:

$$
\begin{aligned}
\Phi_{z}(\bar{z})= & \int_{z=\bar{z}} d^{2} \mathbf{r}\left\langle T_{z z}\right\rangle_{\mathrm{sym}} \\
= & \epsilon_{0} \sum_{\phi \phi^{\prime}} \sum_{p p^{\prime}} \int \frac{d^{2} \mathbf{k}}{(2 \pi)^{2}} \int_{0}^{+\infty} \frac{d \omega}{2 \pi} \exp \left[i\left(\phi k_{z}-\phi^{\prime} k_{z}^{*}\right) \bar{z}\right] \\
& \times\left\{\left(\hat{\epsilon}_{p}^{\phi}\right)_{z}\left(\hat{\epsilon}_{p^{\prime}}^{\phi^{\prime} *}\right)_{z}-\left(\hat{\epsilon}_{p}^{\phi}\right)_{x}\left(\hat{\epsilon}_{p^{\prime}}^{\phi^{\prime} *}\right)_{x}-\left(\hat{\epsilon}_{p}^{\phi}\right)_{y}\left(\hat{\epsilon}_{p^{\prime}}^{\phi^{\prime} *}\right)_{y}\right. \\
& +(-1)^{p+p^{\prime}}\left[\left(\hat{\epsilon}_{S(p)}^{\phi}\right)_{z}\left(\hat{\epsilon}_{S\left(p^{\prime}\right)}^{\phi^{\prime} *}\right)_{z}-\left(\hat{\epsilon}_{S(p)}^{\phi}\right)_{x}\left(\hat{\epsilon}_{S\left(p^{\prime}\right)}^{\phi^{\prime} *}\right)_{x}\right. \\
& \left.\left.-\left(\hat{\epsilon}_{S(p)}^{\phi}\right)_{y}\left(\hat{\epsilon}_{S\left(p^{\prime}\right)}^{\phi^{\prime} *}\right)_{y}\right]\right\}\left\langle p, \mathbf{k}\left|C^{\phi \phi^{\prime}}\right| p^{\prime}, \mathbf{k}\right\rangle,
\end{aligned}
$$

where all the polarization unit vectors are calculated in $(\mathbf{k}, \omega)$. For the other two components of the stress tensor $(m=x, y)$ we have, integrating Eq. (18),

$$
\begin{aligned}
\Phi_{m}(\bar{z})= & \int_{z=\bar{z}} d^{2} \mathbf{r}\left\langle T_{m z}\right\rangle_{\mathrm{sym}} \\
= & \epsilon_{0} \sum_{\phi \phi^{\prime}} \sum_{p p^{\prime}} \int \frac{d^{2} \mathbf{k}}{(2 \pi)^{2}} \int_{0}^{+\infty} \frac{d \omega}{2 \pi} \exp \left[i\left(\phi k_{z}-\phi^{\prime} k_{z}^{*}\right) \bar{z}\right] \\
& \times\left\{\left[\left(\hat{\epsilon}_{p}^{\phi}\right)_{m}\left(\hat{\epsilon}_{p^{\prime}}^{\phi^{\prime} *}\right)_{z}+(-1)^{p+p^{\prime}}\left(\hat{\epsilon}_{S(p)}^{\phi}\right)_{m}\left(\hat{\epsilon}_{S\left(p^{\prime}\right)}^{\phi^{\prime} *}\right)_{z}\right]\right. \\
& +[m \rightleftarrows z]\}\left\langle p, \mathbf{k}\left|C^{\phi \phi^{\prime}}\right| p^{\prime}, \mathbf{k}\right\rangle .
\end{aligned}
$$

From the definitions (7) of the polarization unit vectors we deduce that

$$
\begin{aligned}
& \left(\hat{\epsilon}_{p}^{\phi}\right)_{z}\left(\hat{\epsilon}_{p^{\prime}}^{\phi^{\prime} *}\right)_{z}-\left(\hat{\epsilon}_{p}^{\phi}\right)_{x}\left(\hat{\epsilon}_{p^{\prime}}^{\phi^{\prime} *}\right)_{x}-\left(\hat{\epsilon}_{p}^{\phi}\right)_{y}\left(\hat{\epsilon}_{p^{\prime}}^{\phi^{\prime} *}\right)_{y} \\
& +(-1)^{p+p^{\prime}}\left[\left(\hat{\epsilon}_{S(p)}^{\phi}\right)_{z}\left(\hat{\epsilon}_{S\left(p^{\prime}\right)}^{\phi^{\prime} *}\right)_{z}-\left(\hat{\epsilon}_{S(p)}^{\phi}\right)_{x}\left(\hat{\epsilon}_{S\left(p^{\prime}\right)}^{\phi^{\prime} *}\right)_{x}\right. \\
& \left.-\left(\hat{\epsilon}_{S(p)}^{\phi}\right)_{y}\left(\hat{\epsilon}_{S\left(p^{\prime}\right)}^{\phi^{\prime} *}\right)_{y}\right]=-\delta_{p p^{\prime}} \frac{c^{2}}{\omega^{2}}\left(k_{z}^{2}+\phi \phi^{\prime}\left|k_{z}\right|^{2}\right)
\end{aligned}
$$

and

$$
\begin{aligned}
& {\left[\left(\hat{\epsilon}_{p}^{\phi}\right)_{m}\left(\hat{\epsilon}_{p^{\prime}}^{\phi^{\prime} *}\right)_{z}+(-1)^{p+p^{\prime}}\left(\hat{\epsilon}_{S(p)}^{\phi}\right)_{m}\left(\hat{\epsilon}_{S\left(p^{\prime}\right)}^{\phi^{\prime} *}\right)_{z}\right]+[m \rightleftarrows z]} \\
& \quad=-\delta_{p p^{\prime}} \frac{c^{2} k_{m}}{\omega^{2}}\left(\phi k_{z}+\phi^{\prime} k_{z}^{*}\right) .
\end{aligned}
$$

We now observe that

$$
\begin{gathered}
k_{z}^{2}+\phi \phi^{\prime}\left|k_{z}\right|^{2}= \begin{cases}k_{z}^{2}+\left|k_{z}\right|^{2}, & \phi=\phi^{\prime}, \\
k_{z}^{2}-\left|k_{z}\right|^{2}, & \phi \neq \phi^{\prime},\end{cases} \\
\phi k_{z}+\phi^{\prime} k_{z}^{*}= \begin{cases}2 \phi \operatorname{Re} k_{z}, & \phi=\phi^{\prime}, \\
2 i \phi \operatorname{Im} k_{z}, & \phi \neq \phi^{\prime} .\end{cases}
\end{gathered}
$$

For both expressions, in the former case $\left(\phi=\phi^{\prime}\right)$ only the contribution coming from propagative waves plays a role, while in the latter only evanescent waves are relevant. At the same time, for both expressions (20) and (21), and for both $\phi=\phi^{\prime}$ and $\phi \neq \phi^{\prime}$, the exponential term containing $\bar{z}$ disappears, as expected: whereas the stress tensor depends on $z$, this is not the case for its flux calculated on a plane having an arbitrary position $z=\bar{z}$, provided that $z=\bar{z}$ remains in a given region (A, B, or C). Finally, the flux of $T_{m z}$ on the plane $z=\bar{z}$ can be cast for any $m=x, y, z$ in the form

$$
\begin{aligned}
\Phi_{m}(\bar{z})= & -\sum_{p} \int \frac{d^{2} \mathbf{k}}{(2 \pi)^{2}}\left(\sum_{\phi=\phi^{\prime}} \int_{c k}^{+\infty} \frac{d \omega}{2 \pi}+\sum_{\phi \neq \phi^{\prime}} \int_{0}^{c k} \frac{d \omega}{2 \pi}\right) \\
& \times \frac{2 \epsilon_{0} c^{2} k_{z}}{\omega^{2}}\left\langle p, \mathbf{k}\left|C^{\phi \phi^{\prime}}\right| p, \mathbf{k}\right\rangle \times\left\{\begin{array}{cc}
\phi k_{m}, & m=x, y, \\
k_{z}, & m=z .
\end{array}\right.
\end{aligned}
$$

This equation represents the main result of this section, describing the flux of the component $T_{m z}$ of the stress tensor as a function of the field correlators. As we have shown before, this quantity, together with the explicit knowledge of the correlators in any region of our system which will be discussed in Sec. VI, is sufficient to deduce any component of the force acting on the two bodies. From Eq. (25) we deduce that the flux is written as the sum of two separate contributions, coming from the propagative and evanescent sectors, respectively: the former depends on the correlators between the field propagating in a direction $\phi$ and itself, whilst the latter implies the correlators of counterpropagating fields. We also remark that the quantity $C^{\phi \phi^{\prime}}$ is the only term in Eq. (25) depending on the region in which the flux is calculated, which means on the position of $\bar{z}$. Finally, we observe that the result deduced for the flux of $T_{z z}[m=z$ in Eq. (25)] coincides with the expression obtained in [16].

\section{THE POYNTING VECTOR}

\section{A. General expression for the vector}

Let us now focus our attention on the Poynting vector defined in Eq. (2). In order to evaluate its quantum symmetrized average we first need to work out the generic field product $E_{i} B_{j}$. Using the same conventions as in the last section we obtain

$$
\begin{aligned}
\left(\begin{array}{c}
c E_{i} B_{j} \\
c B_{j} E_{i}
\end{array}\right)= & \sum_{\phi \phi^{\prime}} \sum_{p p^{\prime}} \int \frac{d^{2} \mathbf{k}}{(2 \pi)^{2}} \int \frac{d^{2} \mathbf{k}^{\prime}}{(2 \pi)^{2}} \int_{0}^{+\infty} \frac{d \omega}{2 \pi} \int_{0}^{+\infty} \frac{d \omega^{\prime}}{2 \pi} \\
& \times\left\{\exp \left[i\left(\mathbf{K}^{\phi}-\mathbf{K}^{\prime \phi^{\prime} *}\right) \cdot \mathbf{R}\right] \exp \left[-i\left(\omega-\omega^{\prime}\right) t\right]\right. \\
& \times(-1)^{p^{\prime}}\left(\begin{array}{l}
E_{p}^{\phi}(\mathbf{k}, \omega) E_{p^{\prime}}^{\phi^{\prime} \dagger}\left(\mathbf{k}^{\prime}, \omega^{\prime}\right) \\
E_{p^{\prime}}^{\phi^{\prime} \dagger}\left(\mathbf{k}^{\prime}, \omega^{\prime}\right) E_{p}^{\phi}(\mathbf{k}, \omega)
\end{array}\right)\left[\hat{\epsilon}_{p}^{\phi}(\mathbf{k}, \omega)\right]_{i} \\
& \times\left[\hat{\epsilon}_{S\left(p^{\prime}\right)}^{\phi^{\prime} *}\left(\mathbf{k}^{\prime}, \omega^{\prime}\right)\right]_{j}+\exp \left[i\left(-\mathbf{K}^{\phi *}+\mathbf{K}^{\prime \phi^{\prime}}\right) \cdot \mathbf{R}\right] \\
& \times \exp \left[i\left(\omega-\omega^{\prime}\right) t\right](-1)^{p^{\prime}}\left(\begin{array}{l}
E_{p}^{\phi \dagger}(\mathbf{k}, \omega) E_{p^{\prime}}^{\phi^{\prime}}\left(\mathbf{k}^{\prime}, \omega^{\prime}\right) \\
E_{p^{\prime}}^{\phi^{\prime}}\left(\mathbf{k}^{\prime}, \omega^{\prime}\right) E_{p}^{\phi^{\dagger}}(\mathbf{k}, \omega)
\end{array}\right) \\
& \left.\times\left[\hat{\epsilon}_{p}^{\phi *}(\mathbf{k}, \omega)\right]_{i}\left[\hat{\epsilon}_{S\left(p^{\prime}\right)}^{\phi^{\prime}}\left(\mathbf{k}^{\prime}, \omega^{\prime}\right)\right]_{j}\right\}+\mathcal{Z},
\end{aligned}
$$

from which we immediately get

$$
\begin{aligned}
\left\langle c E_{i} B_{j}\right\rangle_{\mathrm{sym}}= & \sum_{\phi \phi^{\prime}} \sum_{p p^{\prime}} \int \frac{d^{2} \mathbf{k}}{(2 \pi)^{2}} \int \frac{d^{2} \mathbf{k}^{\prime}}{(2 \pi)^{2}} \int_{0}^{+\infty} \frac{d \omega}{2 \pi} \\
& \times \exp \left[i\left(\mathbf{k}-\mathbf{k}^{\prime}\right) \cdot \mathbf{r}\right] \exp \left[i\left(\phi k_{z}-\phi^{\prime} k_{z}^{*}\right) z\right] \\
& \times\left\langle p, \mathbf{k}\left|C^{\phi \phi^{\prime}}\right| p^{\prime}, \mathbf{k}^{\prime}\right\rangle\left\{(-1)^{p^{\prime}}\left[\hat{\epsilon}_{p}^{\phi}(\mathbf{k}, \omega)\right]_{i}\right.
\end{aligned}
$$




$$
\begin{aligned}
& \times\left[\hat{\epsilon}_{S\left(p^{\prime}\right)}^{\phi^{\prime} *}\left(\mathbf{k}^{\prime}, \omega\right)\right]_{j}+(-1)^{p}\left[\hat{\epsilon}_{S(p)}^{\phi}(\mathbf{k}, \omega)\right]_{j} \\
& \left.\times\left[\hat{\epsilon}_{p^{\prime}}^{\phi^{\prime} *}\left(\mathbf{k}^{\prime}, \omega\right)\right]_{i}\right\}
\end{aligned}
$$

with the same conventions as in Eq. (13).

\section{B. Flux of the Poynting vector in terms of field correlators}

We now observe that, by virtue of the same discussion about the closed surface $\Sigma$ given in the last section, we only need the flux of the $z$ component of the Poynting vector in order to evaluate the heat flux on one of the two bodies. For the flux of $S_{z}$ on the plane $z=\bar{z}$ we have

$$
\begin{aligned}
\varphi(\bar{z})= & \int_{z=\bar{z}} d^{2} \mathbf{r}\left\langle S_{z}\right\rangle_{\mathrm{sym}} \\
= & \epsilon_{0} c \sum_{\phi \phi^{\prime}} \sum_{p p^{\prime}} \int \frac{d^{2} \mathbf{k}}{(2 \pi)^{2}} \int_{0}^{+\infty} \frac{d \omega}{2 \pi} \exp \left[i\left(\phi k_{z}-\phi^{\prime} k_{z}^{*}\right) \bar{z}\right] \\
& \times\left\{\left[(-1)^{p^{\prime}}\left(\hat{\epsilon}_{p}^{\phi}\right)_{x}\left(\hat{\epsilon}_{S\left(p^{\prime}\right)}^{\phi^{\prime} *}\right)_{y}+(-1)^{p}\left(\hat{\epsilon}_{S(p)}^{\phi}\right)_{y}\left(\hat{\epsilon}_{p^{\prime}}^{\phi^{\prime} *}\right)_{x}\right]\right. \\
& -[x \rightleftarrows y]\}\left\langle p, \mathbf{k}\left|C^{\phi \phi^{\prime}}\right| p^{\prime}, \mathbf{k}\right\rangle,
\end{aligned}
$$

where we stress the fact that the two terms obtained by interchanging $x$ and $y$ must in this case be changed in sign. We then obtain

$$
\begin{aligned}
& {\left[(-1)^{p^{\prime}}\left(\hat{\epsilon}_{p}^{\phi}\right)_{x}\left(\hat{\epsilon}_{S\left(p^{\prime}\right)}^{\phi^{\prime} *}\right)_{y}+(-1)^{p}\left(\hat{\epsilon}_{S(p)}^{\phi}\right)_{y}\left(\hat{\epsilon}_{p^{\prime}}^{\phi^{\prime} *}\right)_{x}\right]-[x \rightleftarrows y]} \\
& =\delta_{p p^{\prime}} \frac{c}{\omega}\left(\phi k_{z}+\phi^{\prime} k_{z}^{*}\right)
\end{aligned}
$$

and finally cast the expression of the flux of the $z$ component of the Poynting vector in the form

$$
\begin{aligned}
\varphi(\bar{z})= & \sum_{p} \int \frac{d^{2} \mathbf{k}}{(2 \pi)^{2}}\left(\sum_{\phi=\phi^{\prime}} \int_{c k}^{+\infty} \frac{d \omega}{2 \pi}+\sum_{\phi \neq \phi^{\prime}} \int_{0}^{c k} \frac{d \omega}{2 \pi}\right) \\
& \times \frac{2 \epsilon_{0} c^{2} \phi k_{z}}{\omega}\left\langle p, \mathbf{k}\left|C^{\phi \phi^{\prime}}\right| p, \mathbf{k}\right\rangle .
\end{aligned}
$$

This expression is the analog for the Poynting vector of Eq. (25). It constitutes, together with the expression of the matrix elements of $C^{\phi \phi^{\prime}}$ at any frequency, the main ingredient in the calculation of the heat transfer onto the two bodies.

\section{SCATTERING FORMALISM: REFLECTION AND TRANSMISSION OPERATORS}

The quantities $C^{\phi \phi^{\prime}}$ appearing in Eqs. (25) and (30) and defined in Eq. (14) are the correlators of the total fields in each region. In our problem these fields result from the ones emitted by the two bodies and the environmental field, as well as from all the possible scattering processes undergone in the presence of the two bodies 1 and 2 . We then need to introduce a set of operators describing the scattering produced in the presence of a single arbitrary body. Let us suppose we have a body located in the region $z_{1}<z<z_{2}$. Let us further assume that an external field is impinging on our body, either from its left or from its right side. This field will be scattered upon the body, producing in this way new components of the field on both sides of the body. In particular, the field coming from the left (right) will produce a reflected field propagating toward the left (right) on the left (right) side, and a transmitted
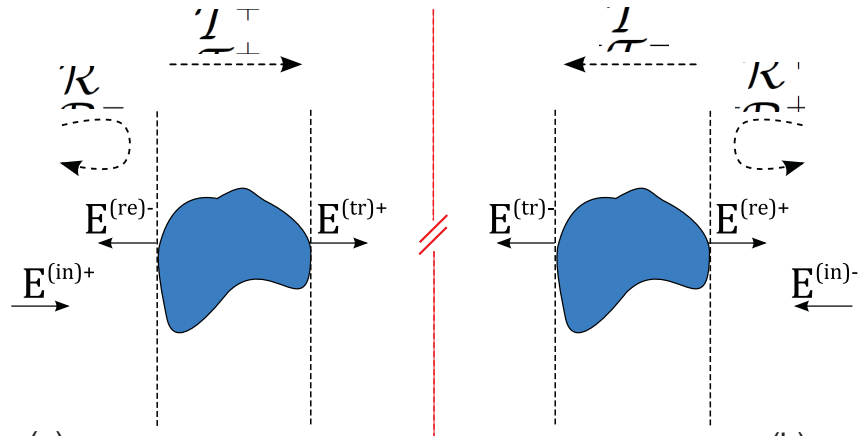

(b)

FIG. 3. (Color online) The definition of the reflection and transmission matrices.

one propagating toward the right (left) on the right (left) side. The two possible configurations, depending on the direction of propagation of the incoming field, are represented in Fig. 3. The reflection and transmission matrices $\mathcal{R}^{ \pm}$and $\mathcal{T}^{ \pm}$are the operators linking each mode of the outgoing fields to the incoming ones.

In particular, considering the case of a field coming from the left side, the incoming field

$$
\begin{aligned}
\mathbf{E}^{(\mathrm{in})+}(\mathbf{R}, t)= & 2 \operatorname{Re}\left[\sum_{p} \int_{0}^{+\infty} \frac{d \omega}{2 \pi} \int \frac{d^{2} \mathbf{k}}{(2 \pi)^{2}} \exp \left[i \mathbf{K}^{+} \cdot \mathbf{R}\right]\right. \\
& \left.\times \exp [-i \omega t] \hat{\boldsymbol{\epsilon}}_{p}^{+}(\mathbf{k}, \omega) E_{p}^{(\mathrm{in})+}(\mathbf{k}, \omega)\right]
\end{aligned}
$$

will result in a reflected (on the left) and a transmitted (on the right) field defined by

$$
\begin{aligned}
\mathbf{E}^{(\mathrm{re})-}(\mathbf{R}, t)= & 2 \operatorname{Re}\left[\sum_{p} \int_{0}^{+\infty} \frac{d \omega}{2 \pi} \int \frac{d^{2} \mathbf{k}}{(2 \pi)^{2}} \exp \left[i \mathbf{K}^{-} \cdot \mathbf{R}\right]\right. \\
& \left.\times \exp [-i \omega t] \hat{\boldsymbol{\epsilon}}_{p}^{-}(\mathbf{k}, \omega) E_{p}^{(\mathrm{re})-}(\mathbf{k}, \omega)\right], \\
\mathbf{E}^{(\mathrm{tr})+}(\mathbf{R}, t)= & 2 \operatorname{Re}\left[\sum_{p} \int_{0}^{+\infty} \frac{d \omega}{2 \pi} \int \frac{d^{2} \mathbf{k}}{(2 \pi)^{2}} \exp \left[i \mathbf{K}^{+} \cdot \mathbf{R}\right]\right. \\
& \left.\times \exp [-i \omega t] \hat{\boldsymbol{\epsilon}}_{p}^{+}(\mathbf{k}, \omega) E_{p}^{(\mathrm{tr})+}(\mathbf{k}, \omega)\right] .
\end{aligned}
$$

As remarked before, since the scattering process is stationary, the frequency is conserved. We are thus able to define the operators $\mathcal{R}^{-}$and $\mathcal{T}^{+}$through the following relations involving the amplitudes defined in Eqs. (31), (32), and (33):

$$
\begin{aligned}
& E_{p}^{(\mathrm{re})-}(\mathbf{k}, \omega)=\sum_{p^{\prime}} \int \frac{d^{2} \mathbf{k}^{\prime}}{(2 \pi)^{2}}\left\langle p, \mathbf{k}\left|\mathcal{R}^{-}\right| p^{\prime}, \mathbf{k}^{\prime}\right\rangle E_{p^{\prime}}^{(\mathrm{in})+}\left(\mathbf{k}^{\prime}, \omega\right) \\
& E_{p}^{(\mathrm{tr})+}(\mathbf{k}, \omega)=\sum_{p^{\prime}} \int \frac{d^{2} \mathbf{k}^{\prime}}{(2 \pi)^{2}}\left\langle p, \mathbf{k}\left|\mathcal{T}^{+}\right| p^{\prime}, \mathbf{k}^{\prime}\right\rangle E_{p^{\prime}}^{(\mathrm{in})+}\left(\mathbf{k}^{\prime}, \omega\right),
\end{aligned}
$$


connecting each mode of the outgoing fields to all the modes of the incoming one at the same frequency $\omega$. In analogy with the field correlators, we are going to drop, for the sake of simplicity, the dependence on the frequency $\omega$ in the reflection and transmission operators. A perfectly analogous procedure leads to the definition of the scattering operators $\mathcal{R}^{-}$and $\mathcal{T}^{+}$.

Using the formalism we have just introduced, the case of the absence of a given body is obtained, as far as its scattering operators are concerned, by imposing

$$
\mathcal{R}^{\phi}=0, \quad \mathcal{T}^{\phi}=1 .
$$

As we will see later, it is convenient to introduce a modified transmission operator which, in analogy with the reflection operator, goes to zero as well in the absence of the body. We thus define

$$
\mathcal{T}^{\phi}=1+\tilde{\mathcal{T}}^{\phi}
$$

writing the transmission operator as the sum of the identity describing the incoming field propagating unmodified on the other side of the body and of a new operator $\tilde{\mathcal{T}}^{\phi}$ accounting only for the scattered part of the field. In the limit of the absence of the body, we have as desired $\tilde{\mathcal{T}}^{\phi}=0$.

\section{FIELD CORRELATORS}

In order to proceed further and to calculate the force and heat transfer on body 1 , we need an expression for the correlators $\left\langle p, \mathbf{k}\left|C^{\phi \phi^{\prime}}\right| p^{\prime}, \mathbf{k}^{\prime}\right\rangle$, defined in Eq. (14), in each region of our system. These correlators will be expressed as a function of the correlators of the field emitted by each body by means of the scattering operators introduced in Sec. V. In this section we will first address the characterization of the single-body field correlators (with this expression we refer both to the bodies 1 and 2 and to the environment) and immediately after express the total-field correlators as a function of these quantities through the scattering operators. We will denote by $E_{p}^{(\gamma) \phi}(\mathbf{k}, \omega)$ each mode of the total field propagating in the direction $\phi$ in the region $\gamma=\mathrm{A}, \mathrm{B}, \mathrm{C}$, as shown in Fig. 1. In order to calculate the fluxes (25) and (30) in the three regions, we need to know the expression for the the correlators $C_{\gamma}^{\phi \phi^{\prime}}$ defined by

$$
\begin{aligned}
\left\langle E_{p}^{(\gamma) \phi}(\mathbf{k}, \omega) E_{p^{\prime}}^{(\gamma) \phi^{\prime} \dagger}\left(\mathbf{k}^{\prime}, \omega^{\prime}\right)\right\rangle_{\mathrm{sym}}= & 2 \pi \delta\left(\omega-\omega^{\prime}\right) \\
& \times\left\langle p, \mathbf{k}\left|C_{\gamma}^{\phi \phi^{\prime}}\right| p^{\prime}, \mathbf{k}^{\prime}\right\rangle .
\end{aligned}
$$

For a system like the one in Fig. 1 at thermal equilibrium at temperature $T$ with the environment, the correlators of the total electromagnetic field outside the body follow from the fluctuation-dissipation theorem [36]:

$$
\begin{aligned}
\left\langle E_{i}^{(\mathrm{tot})}(\mathbf{R}, \omega) E_{j}^{(\mathrm{tot}) \dagger}\left(\mathbf{R}^{\prime}, \omega^{\prime}\right)\right\rangle_{\mathrm{sym}}= & 2 \pi \delta\left(\omega-\omega^{\prime}\right) \frac{2}{\omega} N(\omega, T) \\
& \times \operatorname{Im} G_{i j}\left(\mathbf{R}, \mathbf{R}^{\prime}, \omega\right)
\end{aligned}
$$

where

$$
N(\omega, T)=\frac{\hbar \omega}{2} \operatorname{coth}\left(\frac{\hbar \omega}{2 k_{B} T}\right)=\hbar \omega\left[\frac{1}{2}+n(\omega, T)\right]
$$

with

$$
n(\omega, T)=\frac{1}{e^{\hbar \omega / k_{B} T}-1} .
$$

In Eq. (39) $i$ and $j$ refer to the Cartesian components of the field and $G_{i j}\left(\mathbf{R}, \mathbf{R}^{\prime}, \omega\right)$ is the $i j$ component of the Green function of the system, the solution of the differential equation (see also Appendix C)

$$
\left[\nabla_{\mathbf{R}} \times \nabla_{\mathbf{R}}-\frac{\omega^{2}}{c^{2}} \epsilon(\omega, \mathbf{R})\right] \mathbb{G}\left(\mathbf{R}, \mathbf{R}^{\prime}, \omega\right)=\frac{\omega^{2}}{\epsilon_{0} c^{2}} \mathbb{I} \delta\left(\mathbf{R}-\mathbf{R}^{\prime}\right),
$$

where $\mathbb{I}$ is the identity dyad and $\epsilon(\omega, \mathbf{R})$ the dielectric function of the medium. The property (39) does not hold in the case of a general nonequilibrium configuration. In our particular system, we have assumed that for each body a local temperature can be defined and remains constant in time. This assumption reasonably leads to the hypothesis that the part of the total field emitted by each body is the same as it would be if the body were at thermal equilibrium with the environment at its own temperature. In other words, the emission process is not much influenced by the modification of the external radiation impinging on the body. This hypothesis implies that the correlators of the field emitted by each body can still be deduced using the fluctuation-dissipation theorem Eq. (39) at its local temperature. We note that the limits of validity of this hypothesis, already used in $[1,9,13,16,37]$, require further experimental and theoretical investigations.

We are now ready to write down the expressions for the correlators of the environmental field as well as the fields emitted by the bodies at local thermal equilibrium.

\section{A. Correlators of radiating bodies and environment}

\section{Environmental field}

The correlators of the environmental radiation in equilibrium at temperature $T_{3}$ are well known: they are given, for $\phi, \phi^{\prime} \in\{+,-\}$, by

$$
\begin{aligned}
& \left\langle E_{p}^{(3) \phi}(\mathbf{k}, \omega) E_{p^{\prime}}^{(3) \phi^{\prime} \dagger}\left(\mathbf{k}^{\prime}, \omega^{\prime}\right)\right\rangle_{\text {sym }} \\
& \quad=\delta_{\phi, \phi^{\prime}} \frac{\omega}{2 \epsilon_{0} c^{2}} N\left(\omega, T_{3}\right) \operatorname{Re}\left(\frac{1}{k_{z}}\right) \delta_{p p^{\prime}}(2 \pi)^{3} \delta\left(\omega-\omega^{\prime}\right) \delta\left(\mathbf{k}-\mathbf{k}^{\prime}\right) \\
& \quad=\delta_{\phi, \phi^{\prime}} \frac{\omega}{2 \epsilon_{0} c^{2}} N\left(\omega, T_{3}\right) 2 \pi \delta\left(\omega-\omega^{\prime}\right)\left\langle p, \mathbf{k}\left|\mathcal{P}_{-1}^{(\mathrm{pw})}\right| p^{\prime}, \mathbf{k}^{\prime}\right\rangle \\
& \quad=\delta_{\phi, \phi^{\prime}} 2 \pi \delta\left(\omega-\omega^{\prime}\right)\left\langle p, \mathbf{k}\left|C^{(3)}\right| p^{\prime}, \mathbf{k}^{\prime}\right\rangle .
\end{aligned}
$$

In this expression we have defined the matrix $C^{(3)}$ and introduced the notation, valid for any integer $n$,

$$
\left\langle p, \mathbf{k}\left|\mathcal{P}_{n}^{(\mathrm{pw} / \mathrm{ew})}\right| p^{\prime}, \mathbf{k}^{\prime}\right\rangle=k_{z}^{n}\left\langle p, \mathbf{k}\left|\Pi^{(\mathrm{pw} / \mathrm{ew})}\right| p^{\prime}, \mathbf{k}^{\prime}\right\rangle,
$$

$\Pi^{(\mathrm{pw})}\left(\Pi^{(\mathrm{ew})}\right)$ being the projector on the propagative (evanescent) sector. We remark that the operators $\mathcal{P}_{n}^{\text {(pw/ew) }}$ and $\Pi^{\text {(pw/ew) }}$ depend implicitly on the frequency $\omega$.

\section{Field emitted by each body}

We now calculate the correlators of the field emitted by body $i(i=1,2)$ at temperature $T_{i}$ by ignoring the presence of the other body and assuming thermal equilibrium at temperature $T_{i}$. The main point of this derivation is the connection between the Green function and the scattering operators: the details of this calculation are presented in Appendixes C and D. The 
result for the field correlators is, for two modes of the field propagating in the same direction,

$$
\begin{aligned}
& \left\langle E_{p}^{(i) \phi}(\mathbf{k}, \omega) E_{p^{\prime}}^{(i) \phi^{\dagger}}\left(\mathbf{k}^{\prime}, \omega^{\prime}\right)\right\rangle_{\mathrm{sym}} \\
& =\frac{\omega}{2 \epsilon_{0} c^{2}} N\left(\omega, T_{i}\right) 2 \pi \delta\left(\omega-\omega^{\prime}\right)\langle p, \mathbf{k}| \\
& \quad \times\left(\mathcal{P}_{-1}^{(\mathrm{pw})}-\mathcal{R}^{(i) \phi} \mathcal{P}_{-1}^{(\mathrm{pw})} \mathcal{R}^{(i) \phi^{\dagger}}+\mathcal{R}^{(i) \phi} \mathcal{P}_{-1}^{(\mathrm{ew})}\right. \\
& \left.\quad-\mathcal{P}_{-1}^{(\mathrm{ew})} \mathcal{R}^{(i) \phi \dagger}-\mathcal{T}^{(i) \phi} \mathcal{P}_{-1}^{(\mathrm{pw})} \mathcal{T}^{(i) \phi^{\dagger}}\right)\left|p^{\prime}, \mathbf{k}^{\prime}\right\rangle
\end{aligned}
$$

where $\mathcal{R}^{(i) \phi}$ and $\mathcal{T}^{(i) \phi}$ are respectively the reflection and transmission operators associated with the side $\phi$ of body $i$ defined in Sec. V. For fields propagating in opposite directions $\left(\phi \neq \phi^{\prime}\right)$, we have

$$
\begin{aligned}
& \left\langle E_{p}^{(i) \phi}(\mathbf{k}, \omega) E_{p^{\prime}}^{(i) \phi^{\prime} \dagger}\left(\mathbf{k}^{\prime}, \omega^{\prime}\right)\right\rangle_{\mathrm{sym}} \\
& =\frac{\omega}{2 \epsilon_{0} c^{2}} N\left(\omega, T_{i}\right) 2 \pi \delta\left(\omega-\omega^{\prime}\right)\langle p, \mathbf{k}| \\
& \quad \times\left(-\mathcal{R}^{(i) \phi} \mathcal{P}_{-1}^{(\mathrm{pw})} \mathcal{T}^{(i) \phi^{\prime} \dagger}-\mathcal{T}^{(i) \phi} \mathcal{P}_{-1}^{(\mathrm{pw})} \mathcal{R}^{(i) \phi^{\prime} \dagger}\right. \\
& \left.\quad+\mathcal{T}^{(i) \phi} \mathcal{P}_{-1}^{(\mathrm{ew})}-\mathcal{P}_{-1}^{(\mathrm{ew})} \mathcal{T}^{(i) \phi^{\prime} \dagger}\right)\left|p^{\prime}, \mathbf{k}^{\prime}\right\rangle
\end{aligned}
$$

In analogy with the previous definitions, the correlators of the field produced by the body $i$ will be gathered in the matrix $C^{(i) \phi \phi^{\prime}}$, defined by the relation

$$
\begin{aligned}
\left\langle E_{p}^{(i) \phi}(\mathbf{k}, \omega) E_{p^{\prime}}^{(i) \phi^{\prime} \dagger}\left(\mathbf{k}^{\prime}, \omega^{\prime}\right)\right\rangle_{\mathrm{sym}}= & 2 \pi \delta\left(\omega-\omega^{\prime}\right) \\
& \times\left\langle p, \mathbf{k}\left|C^{(i) \phi \phi^{\prime}}\right| p^{\prime}, \mathbf{k}^{\prime}\right\rangle .
\end{aligned}
$$

We are now ready to characterize the total field in each region by means of the scattering operators and then to deduce its correlators using the results just obtained in this section.

\section{B. Correlators in region $B$}

In order to build up the field in the region $B$ between the two bodies the ingredients we need are the amplitudes $E_{p}^{(1)+}(\mathbf{k}, \omega)$, $E_{p}^{(2)-}(\mathbf{k}, \omega)$, and $E_{p}^{(3) \pm}(\mathbf{k}, \omega)$. In the region B of Fig. 1 the field propagates in both directions: its amplitudes will be simply denoted by $E_{p}^{(\mathrm{B}) \phi}(\mathbf{k}, \omega)$. Gathering all the modes $E_{p}^{(\mathrm{B}) \phi}(\mathbf{k}, \omega)$ in the symbol $E^{(\mathrm{B}) \phi}$, the amplitudes can be expressed as the solutions of the system of equations

$$
\left\{\begin{array}{l}
E^{(\mathrm{B})+}=E^{(1)+}+\mathcal{T}^{(1)+} E^{(3)+}+\mathcal{R}^{(1)+} E^{(\mathrm{B})-}, \\
E^{(\mathrm{B})-}=E^{(2)-}+\mathcal{T}^{(2)-} E^{(3)-}+\mathcal{R}^{(2)-} E^{(\mathrm{B})+},
\end{array}\right.
$$

where all the operators and field amplitudes are calculated at a given frequency $\omega$, not explicitly indicated, and the products between scattering operators and fields are to be considered as matrix-vector products. As an intermediate step, we have

$$
\left\{\begin{aligned}
E^{(\mathrm{B})+}= & E^{(1)+}+\mathcal{T}^{(1)+} E^{(3)+}+\mathcal{R}^{(1)+} E^{(\mathrm{B})-}, \\
E^{(\mathrm{B})-}= & U^{(21)} \mathcal{R}^{(2)-} E^{(1)+}+U^{(21)} \mathcal{R}^{(2)-} \mathcal{T}^{(1)+} E^{(3)+} \\
& +U^{(21)} \mathcal{T}^{(2)-} E^{(3)-}+U^{(21)} E^{(2)-}
\end{aligned}\right.
$$

where we have introduced the operators

$$
\begin{aligned}
& U^{(12)}=\left(1-\mathcal{R}^{(1)+} \mathcal{R}^{(2)-}\right)^{-1}, \\
& U^{(21)}=\left(1-\mathcal{R}^{(2)-} \mathcal{R}^{(1)+}\right)^{-1}
\end{aligned}
$$

describing the series of intracavity (between the two bodies) reflections produced by the single-body operators $\mathcal{R}^{(1)+}$ and $\mathcal{R}^{(2)-}$. From the definition

$$
U^{(12)}=\left(1-\mathcal{R}^{(1)+} \mathcal{R}^{(2)-}\right)^{-1}=\sum_{n=0}^{+\infty}\left(\mathcal{R}^{(1)+} \mathcal{R}^{(2)-}\right)^{n}
$$

and its analogous counterpart for $U^{(21)}$, we easily deduce the following useful properties:

$$
\mathcal{R}^{(1)+} U^{(21)}=U^{(12)} \mathcal{R}^{(1)+}, \quad \mathcal{R}^{(2)-} U^{(12)}=U^{(21)} \mathcal{R}^{(2)-},
$$

and

$$
\begin{aligned}
& \mathcal{R}^{(1)+} U^{(21)} \mathcal{R}^{(2)-}=U^{(12)}-1, \\
& \mathcal{R}^{(2)-} U^{(12)} \mathcal{R}^{(1)+}=U^{(21)}-1 .
\end{aligned}
$$

These relations allow us to obtain the following final expression for the field propagating in both directions in region $\mathrm{B}$ as a function of the fields emitted by the bodies and the environment:

$$
\left\{\begin{aligned}
E^{(\mathrm{B})+}= & U^{(12)} E^{(1)+}+\mathcal{R}^{(1)+} U^{(21)} E^{(2)-} \\
& +\mathcal{R}^{(1)+} U^{(21)} \mathcal{T}^{(2)-} E^{(3)-}+U^{(12)} \mathcal{T}^{(1)+} E^{(3)+} \\
E^{(\mathrm{B})-}= & \mathcal{R}^{(2)-} U^{(12)} E^{(1)+}+U^{(21)} E^{(2)-} \\
& +U^{(21)} \mathcal{T}^{(2)-} E^{(3)-}+\mathcal{R}^{(2)-} U^{(12)} \mathcal{T}^{(1)+} E^{(3)+}
\end{aligned}\right.
$$

We remark here that by taking $E^{(3) \pm}=0$ or $\mathcal{T}^{(1)+}=\mathcal{T}^{(2)-}=0$ in Eq. (54) we go back to Eqs. (18) and (19) of [16], where for both bodies infinite thickness was assumed. Since the fields $E^{(1)-}$ and $E^{(2)+}$ clearly do not participate in the expression of the total field between the two bodies, the expression of $E^{(\mathrm{B}) \phi}$ (for $\phi=+,-$ ) can be cast without loss of generality in the form

$$
E^{(\mathrm{B}) \pm}=A_{1}^{(\mathrm{B}) \pm} E^{(1)+}+A_{2}^{(\mathrm{B}) \pm} E^{(2)-}+\sum_{\alpha=+,-} B_{\alpha}^{(\mathrm{B}) \pm} E^{(3) \alpha},
$$

which in this case gives, by comparison with Eq. (54),

$$
\begin{gathered}
A_{1}^{(\mathrm{B})+}=U^{(12)}, \quad A_{2}^{(\mathrm{B})+}=\mathcal{R}^{(1)+} U^{(21)}, \\
B_{-}^{(\mathrm{B})+}=\mathcal{R}^{(1)+} U^{(21)} \mathcal{T}^{(2)-}, \quad B_{+}^{(\mathrm{B})+}=U^{(12)} \mathcal{T}^{(1)+}, \\
A_{1}^{(\mathrm{B})-}=\mathcal{R}^{(2)-} U^{(12)}, \quad A_{2}^{(\mathrm{B})-}=U^{(21)}, \\
B_{-}^{(\mathrm{B})-}=U^{(21)} \mathcal{T}^{(2)-}, \quad B_{+}^{(\mathrm{B})-}=\mathcal{R}^{(2)-} U^{(12)} \mathcal{T}^{(1)+} .
\end{gathered}
$$

Using Eq. (55) the correlators in region B can be expressed as a function of the correlators $C^{(i) \phi \phi}$ (for $i=1,2$ ) and $C^{(3)}$ given by Eqs. (43), (45) and (46). We finally obtain the expression for the matrix $C_{\mathrm{B}}^{\phi \phi^{\prime}}$ in terms of the scattering operators of the two bodies:

$$
\begin{aligned}
& \left\langle E_{p}^{(\mathrm{B}) \phi}(\mathbf{k}, \omega) E_{p^{\prime}}^{(\mathrm{B}) \phi^{\prime} \dagger}\left(\mathbf{k}^{\prime}, \omega^{\prime}\right)\right\rangle_{\mathrm{sym}} \\
& =2 \pi \delta\left(\omega-\omega^{\prime}\right)\langle p, \mathbf{k}|\left[A_{1}^{(\mathrm{B}) \phi} C^{(1)++} A_{1}^{(\mathrm{B}) \phi^{\prime} \dagger}\right. \\
& \left.\quad+A_{2}^{(\mathrm{B}) \phi} C^{(2)--} A_{2}^{(\mathrm{B}) \phi^{\prime} \dagger}+\sum_{\alpha=+,-} B_{\alpha}^{(\mathrm{B}) \phi} C^{(3)} B_{\alpha}^{(\mathrm{B}) \phi^{\prime} \dagger}\right]\left|p^{\prime}, \mathbf{k}^{\prime}\right\rangle \\
& =2 \pi \delta\left(\omega-\omega^{\prime}\right)\left\langle p, \mathbf{k}\left|C_{\mathrm{B}}^{\phi \phi^{\prime}}\right| p^{\prime}, \mathbf{k}^{\prime}\right\rangle .
\end{aligned}
$$




\section{Correlators in region $\mathbf{A}$}

The complete knowledge of the properties of the field in region $\mathrm{B}$ is not sufficient, in general, to deduce the force and the heat transfer associated with either of the two bodies. Focusing our attention on body 1, for example, we also need to characterize the field in the region on its left side, namely, region $\mathrm{A}$. The field $E^{(\mathrm{A})+}$ propagating toward the right in this region is obviously only the environmental field propagating in the same direction, $E^{(3)+}$. In contrast, as far as the field $E^{(\mathrm{A})-}$ is concerned, it will also include, assuming a finite thickness for body 1, components from the fields produced by bodies 1 and 2 , as well as from the environment field $E^{(3)-}$. The total field in region $\mathrm{A}$ is then entirely described by the system of equations

$$
\left\{\begin{array}{l}
E^{(\mathrm{A})+}=E^{(3)+} \\
E^{(\mathrm{A})-}=E^{(1)-}+\mathcal{R}^{(1)-} E^{(3)+}+\mathcal{T}^{(1)-} E^{(\mathrm{B})-},
\end{array}\right.
$$

which using the result (54) for the intracavity field becomes

$$
\left\{\begin{aligned}
E^{(\mathrm{A})+}= & E^{(3)+} \\
E^{(\mathrm{A})-}= & E^{(1)-}+\mathcal{T}^{(1)-} \mathcal{R}^{(2)-} U^{(12)} E^{(1)+} \\
& +\mathcal{T}^{(1)-} U^{(21)} E^{(2)-}+\mathcal{T}^{(1)-} U^{(21)} \mathcal{T}^{(2)-} E^{(3)-} \\
& +\left(\mathcal{R}^{(1)-}+\mathcal{T}^{(1)-} \mathcal{R}^{(2)-} U^{(12)} \mathcal{T}^{(1)+}\right) E^{(3)+}
\end{aligned}\right.
$$

Using the general decomposition

$$
E^{(\mathrm{A})-}=\sum_{\alpha=+,-} A_{1 \alpha}^{(\mathrm{A})} E^{(1) \alpha}+A_{2}^{(\mathrm{A})} E^{(2)-}+\sum_{\alpha=+,-} B_{\alpha}^{(\mathrm{A})} E^{(3) \alpha},
$$

we have

$$
\begin{aligned}
A_{1+}^{(\mathrm{A})}= & \mathcal{T}^{(1)-} \mathcal{R}^{(2)-} U^{(12)}, \quad A_{1-}^{(\mathrm{A})}=1, \\
& A_{2}^{(\mathrm{A})}=\mathcal{T}^{(1)-} U^{(21)}, \\
B_{+}^{(\mathrm{A})}= & \mathcal{R}^{(1)-}+\mathcal{T}^{(1)-} \mathcal{R}^{(2)-} U^{(12)} \mathcal{T}^{(1)+}, \\
& B_{-}^{(\mathrm{A})}=\mathcal{T}^{(1)-} U^{(21)} \mathcal{T}^{(2)-} .
\end{aligned}
$$

We are now ready to give the final expression for the correlators $C_{A}^{\phi \phi^{\prime}}$ of the total field in region $\mathrm{A}$ in terms of the scattering matrices, which reads

$$
\begin{gathered}
C_{A}^{++}=C^{(3)}, \\
C_{A}^{+-}=C^{(3)} B_{+}^{(\mathrm{A}) \dagger}, \\
C_{A}^{-+}=B_{+}^{(\mathrm{A})} C^{(3)} \\
C_{A}^{--}=A_{1+}^{(\mathrm{A})} C^{(1)++} A_{1+}^{(\mathrm{A}) \dagger}+C^{(1)--}+A_{1+}^{(\mathrm{A})} C^{(1)+-} \\
+C^{(1)-+} A_{1+}^{(\mathrm{A}) \dagger}+A_{2}^{(\mathrm{A})} C^{(2)--} A_{2}^{(\mathrm{A}) \dagger}+B_{+}^{(\mathrm{A})} C^{(3)} B_{+}^{(\mathrm{A}) \dagger} \\
+B_{-}^{(\mathrm{A})} C^{(3)} B_{-}^{(\mathrm{A}) \dagger} .
\end{gathered}
$$

Due to the geometry of our system, the correlators of the field in region $\mathrm{C}$ can be obtained from the ones given here for region A performing the interchanges $\mathrm{A} \rightleftarrows \mathrm{C}, 1 \rightleftarrows 2$ and $+\rightleftarrows-$. This holds for all the other quantities we are going to calculate in region $\mathrm{A}$.

\section{FINAL EXPRESSIONS FOR THE CASIMIR-LIFSHITZ FORCE AND HEAT TRANSFER OUT OF THERMAL EQUILIBRIUM}

\section{A. Casimir-Lifshitz force}

We are now going to calculate the flux of the stress tensor, in order to deduce the expression of the force. For simplicity, we will focus on the $z$ component of the force acting on body 1 , using as a consequence the expression (25) with $m=z$ in regions $\mathrm{B}$ and $\mathrm{A}$. The calculation of the other components of the force follows the same scheme we are going to present in the following. Let us now turn, then, to the evaluation of the fluxes of $T_{z z}$ in regions B (Sec. VII A 1) and A (Sec. VII A 2): we will assume that for both surfaces the vector orthogonal to the surface is oriented toward the right, i.e., the positive direction of the $z$ axis. These two results will provide the final expression for the Casimir-Lifshitz force acting on body 1, deduced in Sec. VII A 3.

\section{Flux in region $B$}

Using the relation (25) (with $m=z$ and $z_{2}<\bar{z}<z_{3}$ ) and (57), we are able to express the flux in region $B$ as a function of the correlators of the field emitted by the bodies and the environment (43), (45), and (46),

$$
\begin{aligned}
& \Phi_{z}^{(\mathrm{B})}\left(T_{1}, T_{2}, T_{3}\right) \\
& =-2 \epsilon_{0} \sum_{p} \int \frac{d^{2} \mathbf{k}}{(2 \pi)^{2}} \int_{0}^{+\infty} \frac{d \omega}{2 \pi} \frac{c^{2} k_{z}^{2}}{\omega^{2}}\langle p, \mathbf{k}| \\
& \times\left\{\Pi ^ { ( \mathrm { pw } ) } \left(A_{1}^{(\mathrm{B})+} C^{(1)++} A_{1}^{(\mathrm{B})+\dagger}\right.\right. \\
& +A_{1}^{(\mathrm{B})-} C^{(1)++} A_{1}^{(\mathrm{B})-\dagger}+A_{2}^{(\mathrm{B})+} C^{(2)--} A_{2}^{(\mathrm{B})+\dagger} \\
& \left.+A_{2}^{(\mathrm{B})-} C^{(2)--} A_{2}^{(\mathrm{B})-\dagger}\right) \\
& +\Pi^{(\mathrm{ew})}\left(A_{1}^{(\mathrm{B})+} C^{(1)++} A_{1}^{(\mathrm{B})-\dagger}+A_{1}^{(\mathrm{B})-} C^{(1)++} A_{1}^{(\mathrm{B})+\dagger}\right. \\
& \left.+A_{2}^{(\mathrm{B})+} C^{(2)--} A_{2}^{(\mathrm{B})-\dagger}+A_{2}^{(\mathrm{B})-} C^{(2)--} A_{2}^{(\mathrm{B})+\dagger}\right) \\
& +\sum_{\alpha=+,-}\left[\Pi^{(\mathrm{pw})}\left(B_{\alpha}^{(\mathrm{B})+} C^{(3)} B_{\alpha}^{(\mathrm{B})+\dagger}+B_{\alpha}^{(\mathrm{B})-} C^{(3)} B_{\alpha}^{(\mathrm{B})-\dagger}\right)\right. \\
& \left.\left.+\Pi^{(\mathrm{ew})}\left(B_{\alpha}^{(\mathrm{B})+} C^{(3)} B_{\alpha}^{(\mathrm{B})-\dagger}+B_{\alpha}^{(\mathrm{B})-} C^{(3)} B_{\alpha}^{(\mathrm{B})+\dagger}\right)\right]\right\}|p, \mathbf{k}\rangle .
\end{aligned}
$$

Defining the trace operator for a frequency-dependent operator $\mathcal{A}$ as

$$
\operatorname{Tr} \mathcal{A}=\sum_{p} \int \frac{d^{2} \mathbf{k}}{(2 \pi)^{2}} \int_{0}^{+\infty} \frac{d \omega}{2 \pi}\langle p, \mathbf{k}|\mathcal{A}| p, \mathbf{k}\rangle,
$$

we can write the flux in region $B$ in the form

$$
\begin{aligned}
\Phi_{z}^{(\mathrm{B})} & \left(T_{1}, T_{2}, T_{3}\right) \\
= & -2 \epsilon_{0} c^{2} \operatorname{Tr}\left\{\frac { 1 } { \omega ^ { 2 } } \left[\mathcal { P } _ { 2 } ^ { ( \mathrm { pw } ) } \left(A_{1}^{(\mathrm{B})+} C^{(1)++} A_{1}^{(\mathrm{B})+\dagger}\right.\right.\right. \\
& +A_{1}^{(\mathrm{B})-} C^{(1)++} A_{1}^{(\mathrm{B})-\dagger}+A_{2}^{(\mathrm{B})+} C^{(2)--} A_{2}^{(\mathrm{B})+\dagger} \\
& \left.+A_{2}^{(\mathrm{B})-} C^{(2)--} A_{2}^{(\mathrm{B})-\dagger}\right)+\mathcal{P}_{2}^{(\mathrm{ew})}\left(A_{1}^{(\mathrm{B})+} C^{(1)++} A_{1}^{(\mathrm{B})-\dagger}\right. \\
& +A_{1}^{(\mathrm{B})-} C^{(1)++} A_{1}^{(\mathrm{B})+\dagger}+A_{2}^{(\mathrm{B})+} C^{(2)--} A_{2}^{(\mathrm{B})-\dagger} \\
& \left.+A_{2}^{(\mathrm{B})-} C^{(2)--} A_{2}^{(\mathrm{B})+\dagger}\right)
\end{aligned}
$$




$$
\begin{aligned}
& +\sum_{\alpha=+,-}\left[\mathcal{P}_{2}^{(\mathrm{pw})}\left(B_{\alpha}^{(\mathrm{B})+} C^{(3)} B_{\alpha}^{(\mathrm{B})+\dagger}+B_{\alpha}^{(\mathrm{B})-} C^{(3)} B_{\alpha}^{(\mathrm{B})-\dagger}\right)\right. \\
& \left.\left.\left.+\mathcal{P}_{2}^{(\mathrm{ew})}\left(B_{\alpha}^{(\mathrm{B})+} C^{(3)} B_{\alpha}^{(\mathrm{B})-\dagger}+B_{\alpha}^{(\mathrm{B})-} C^{(3)} B_{\alpha}^{(\mathrm{B})+\dagger}\right)\right]\right]\right\} .
\end{aligned}
$$

In both expressions it is clear that we have three separate contributions associated with body 1 , body 2 , and the environment, respectively. Using the fact that the trace is invariant under cyclic permutations, we have

$$
\begin{aligned}
\Phi_{z}^{(\mathrm{B})} & \left(T_{1}, T_{2}, T_{3}\right) \\
= & -\operatorname{Tr}\left\{\frac { 1 } { \omega } \left[N\left(\omega, T_{1}\right) J\left(\mathcal{R}^{(1)+}, \mathcal{R}^{(2)-}\right)+N\left(\omega, T_{2}\right)\right.\right. \\
& \times J\left(\mathcal{R}^{(2)-}, \mathcal{R}^{(1)+}\right)+\left(N\left(\omega, T_{3}\right)-N\left(\omega, T_{1}\right)\right) \\
& \times H\left(\mathcal{R}^{(1)+}, \mathcal{R}^{(2)-}, \mathcal{T}^{(1)+}\right)+\left(N\left(\omega, T_{3}\right)-N\left(\omega, T_{2}\right)\right) \\
& \left.\left.\times H\left(\mathcal{R}^{(2)-}, \mathcal{R}^{(1)+}, \mathcal{T}^{(2)-}\right)\right]\right\},
\end{aligned}
$$

where

$$
\begin{aligned}
J\left(\mathcal{R}^{(1)+}, \mathcal{R}^{(2)-}\right)= & U^{(12)}\left(\mathcal{P}_{-1}^{(\mathrm{pw})}-\mathcal{R}^{(1)+} \mathcal{P}_{-1}^{(\mathrm{pw})} \mathcal{R}^{(1)+\dagger}\right. \\
& \left.+\mathcal{R}^{(1)+} \mathcal{P}_{-1}^{(\mathrm{ew})}-\mathcal{P}_{-1}^{(\mathrm{ew})} \mathcal{R}^{(1)+\dagger}\right) U^{(12)^{\dagger}} \\
& \times\left(\mathcal{P}_{2}^{(\mathrm{pw})}+\mathcal{R}^{(2)-\dagger} \mathcal{P}_{2}^{(\mathrm{pw})} \mathcal{R}^{(2)-}\right. \\
& \left.+\mathcal{R}^{(2)-\dagger} \mathcal{P}_{2}^{(\mathrm{ew})}+\mathcal{P}_{2}^{(\mathrm{ew})} \mathcal{R}^{(2)-}\right) \\
H\left(\mathcal{R}^{(1)+}, \mathcal{R}^{(2)-}, \mathcal{T}^{(1)+}\right)= & U^{(12)} \mathcal{T}^{(1)+} \mathcal{P}_{-1}^{\mathrm{pw}} \mathcal{T}^{(1)+\dagger} U^{(12) \dagger} \\
& \times\left(\mathcal{P}_{2}^{(\mathrm{pw})}+\mathcal{R}^{(2)-\dagger} \mathcal{P}_{2}^{(\mathrm{pw})} \mathcal{R}^{(2)-}\right. \\
& \left.+\mathcal{R}^{(2)-\dagger} \mathcal{P}_{2}^{(\mathrm{ew})}+\mathcal{P}_{2}^{(\mathrm{ew})} \mathcal{R}^{(2)-}\right) .
\end{aligned}
$$

Note that when calculating $J\left(\mathcal{R}^{(2)-}, \mathcal{R}^{(1)+}\right)$ one also needs to change $U^{(12)}$ into $U^{(21)}$.

Equation (66) can be cast in the form

$$
\begin{aligned}
\Phi_{z}^{(\mathrm{B})}\left(T_{1}, T_{2}, T_{3}\right)= & \frac{\Phi_{z}^{(\mathrm{B}, \text { eq })}\left(T_{1}\right)+\Phi_{z}^{(\mathrm{B}, \text { eq })}\left(T_{2}\right)}{2} \\
& +\Delta \Phi_{z}^{(\mathrm{B})}\left(T_{1}, T_{2}, T_{3}\right),
\end{aligned}
$$

where

$$
\begin{aligned}
\Phi_{z}^{(\mathrm{B}, \mathrm{eq})}(T)= & -\operatorname{Tr}\left\{\frac { 1 } { \omega } N ( \omega , T ) \left[J\left(\mathcal{R}^{(1)+}, \mathcal{R}^{(2)-}\right)\right.\right. \\
& \left.\left.+J\left(\mathcal{R}^{(2)-}, \mathcal{R}^{(1)+}\right)\right]\right\} \\
= & -2 \operatorname{Re} \operatorname{Tr}\left\{\frac{k_{z}}{\omega} N(\omega, T)\right. \\
& \left.\times\left[U^{(12)} \mathcal{R}^{(1)+} \mathcal{R}^{(2)-}+U^{(21)} \mathcal{R}^{(2)-} \mathcal{R}^{(1)+}\right]\right\} \\
& -2 \operatorname{Tr}\left\{\frac{1}{\omega} N(\omega, T) \mathcal{P}_{1}^{\mathrm{pw}}\right\}
\end{aligned}
$$

and

$$
\begin{aligned}
& \Delta \Phi_{z}^{(\mathrm{B})}\left(T_{1}, T_{2}, T_{3}\right) \\
& =-\hbar \operatorname{Tr}\left[\frac{n_{12}}{2}\left(J\left(\mathcal{R}^{(1)+}, \mathcal{R}^{(2)-}\right)-J\left(\mathcal{R}^{(2)-}, \mathcal{R}^{(1)+}\right)\right)\right. \\
& \quad+n_{31} H\left(\mathcal{R}^{(1)+}, \mathcal{R}^{(2)-}, \mathcal{T}^{(1)+}\right) \\
& \left.\quad+n_{32} H\left(\mathcal{R}^{(2)-}, \mathcal{R}^{(1)+}, \mathcal{T}^{(2)-}\right)\right],
\end{aligned}
$$

where we have defined, for $i, j=1,2,3$,

$$
n_{i j}=n\left(\omega, T_{i}\right)-n\left(\omega, T_{j}\right) .
$$

At this point, a remark is important about the expression (69), giving the equilibrium part of the flux in region B of $T_{z z}$. We have to observe that, since the operator $\mathcal{P}_{1}^{(\mathrm{pw})}$ is diagonal in the $(\mathbf{k}, p)$ basis, its trace defined as in (64) is divergent. Moreover, this term is independent of the bodies under scrutiny. Nevertheless, we have to keep in mind that the flux in region B does not have a direct physical meaning, since we still have to subtract from it the flux in region A in order to obtain the value of the force. As we will see in Secs. VII A 3 and VII C, the divergences present in the individual fluxes are completely regularized when taking the difference $\Phi_{z}^{(\mathrm{B})}-\Phi_{z}^{(\mathrm{A})}$.

\section{Flux in region $A$}

In order to calculate the flux of $T_{z z}$ in A we have to use Eq. (25) with $m=z$ and $\bar{z}<z_{1}$. Moreover, the correlators in region $\mathrm{A}$ are given in Eq. (62). After algebraic manipulations analogous to the ones used in the last section we have, for the flux in region $\mathrm{A}$,

$$
\begin{aligned}
\Phi_{z}^{(\mathrm{A})} & \left(T_{1}, T_{2}, T_{3}\right) \\
= & -2 \epsilon_{0} c^{2} \operatorname{Tr}\left[\frac { 1 } { \omega ^ { 2 } } \mathcal { P } _ { 2 } ^ { ( \mathrm { pw } ) } \left(C^{(3)}+A_{1+}^{(\mathrm{A})} C^{(1)++} A_{1+}^{(\mathrm{A}) \dagger}+C^{(1)--}\right.\right. \\
& +A_{1+}^{(\mathrm{A})} C^{(1)+-}+C^{(1)-+} A_{1+}^{(\mathrm{A}) \dagger}+A_{2}^{(\mathrm{A})} C^{(2)--} A_{2}^{(\mathrm{A}) \dagger} \\
& \left.\left.+B_{+}^{(\mathrm{A})} C^{(3)} B_{+}^{(\mathrm{A}) \dagger}+B_{-}^{(\mathrm{A})} C^{(3)} B_{-}^{(\mathrm{A}) \dagger}\right)\right] .
\end{aligned}
$$

This can be cast in the form

$$
\begin{aligned}
\Phi_{z}^{(\mathrm{A})}\left(T_{1}, T_{2}, T_{3}\right)= & -\operatorname{Tr}\left[\frac{1}{\omega}\left(N\left(\omega, T_{1}\right)+N\left(\omega, T_{3}\right)\right) \mathcal{P}_{1}^{(\mathrm{pw})}\right] \\
& +\Delta \Phi_{z}^{(\mathrm{A})}\left(T_{1}, T_{2}, T_{3}\right),
\end{aligned}
$$

where

$$
\begin{aligned}
\Delta \Phi_{z}^{(\mathrm{A})} & \left(T_{1}, T_{2}, T_{3}\right) \\
= & -\hbar \operatorname{Tr}\left[n_{31} \mathcal{P}_{2}^{(\mathrm{pw})} \mathcal{R}^{(1)-} \mathcal{P}_{-1}^{(\mathrm{pw})} \mathcal{R}^{(1)-\dagger}\right. \\
& +n_{32} \mathcal{P}_{2}^{(\mathrm{pw})} \mathcal{T}^{(1)-} U^{(21)} \mathcal{T}^{(2)-} \mathcal{P}_{-1}^{(\mathrm{pw})} \mathcal{T}^{(2)-\dagger} U^{(21) \dagger} \mathcal{T}^{(1)-\dagger} \\
& +n_{21}\left(\mathcal{P}_{-1}^{(\mathrm{pw})}+\mathcal{R}^{(2)-} \mathcal{P}_{-1}^{(\mathrm{ew})}-\mathcal{P}_{-1}^{(\mathrm{ew})} \mathcal{R}^{(2)-\dagger}\right. \\
& \left.-\mathcal{R}^{(2)-} \mathcal{P}_{-1}^{(\mathrm{pw})} \mathcal{R}^{(2)-\dagger}\right) U^{(21) \dagger} \mathcal{T}^{(1)-\dagger} \mathcal{P}_{2}^{(\mathrm{pw})} \mathcal{T}^{(1)-} U^{(21)} \\
& +n_{31}\left(\mathcal{P}_{2}^{(\mathrm{pw})} \mathcal{R}^{(1)-} \mathcal{P}_{-1}^{(\mathrm{pw})} \mathcal{T}^{(1)+\dagger} U^{(12) \dagger} \mathcal{R}^{(2)-\dagger} \mathcal{T}^{(1)-\dagger}\right. \\
& +\mathcal{P}_{2}^{(\mathrm{pw})} \mathcal{T}^{(1)-} \mathcal{R}^{(2)-} U^{(12)} \mathcal{T}^{(1)+} \mathcal{P}_{-1}^{(\mathrm{pw})} \mathcal{R}^{(1)-\dagger} \\
& +\mathcal{P}_{2}^{(\mathrm{pw})} \mathcal{T}^{(1)-} \mathcal{R}^{(2)-} U^{(12)} \mathcal{T}^{(1)+} \mathcal{P}_{-1}^{(\mathrm{pw})} \mathcal{T}^{(1)+\dagger} \\
& \left.\left.\times U^{(12) \dagger} \mathcal{R}^{(2)-\dagger} \mathcal{T}^{(1)-\dagger}\right)\right]
\end{aligned}
$$

We repeat here that the flux in region $C$ (necessary for the calculation of the force acting on body 2) can be obtained from Eqs. (73) and (74) by performing the interchanges $A \rightleftarrows C$, $1 \rightleftarrows 2$, and $+\rightleftarrows-$.

\section{Casimir-Lifshitz force acting on body 1}

We now have all the ingredients to give the $z$ component of the force acting on body 1 . From the definition of the stress 
tensor we have

$$
F_{1 z}=\Phi_{z}^{(\mathrm{B})}\left(T_{1}, T_{2}, T_{3}\right)-\Phi_{z}^{(\mathrm{A})}\left(T_{1}, T_{2}, T_{3}\right),
$$

where the two fluxes are given by Eqs. (68) and (73). Gathering all the results obtained in the previous sections, the complete expression of the force reads

$$
F_{1 z}\left(T_{1}, T_{2}, T_{3}\right)=\frac{F_{z}^{(\mathrm{eq})}\left(T_{1}\right)+F_{z}^{(\mathrm{eq})}\left(T_{2}\right)}{2}+\Delta F_{1 z}\left(T_{1}, T_{2}, T_{3}\right)
$$

In this expression the result is written as a sum of two terms. The first contribution is the average, at the temperatures $T_{1}$ and $T_{2}$ of the two bodies, of the equilibrium force

$$
\begin{aligned}
F_{z}^{(\mathrm{eq})}(T)= & -2 \operatorname{Re} \operatorname{Tr}\left\{\frac { k _ { z } } { \omega } N ( \omega , T ) \left[U^{(12)} \mathcal{R}^{(1)+} \mathcal{R}^{(2)-}\right.\right. \\
& \left.\left.+U^{(21)} \mathcal{R}^{(2)-} \mathcal{R}^{(1)+}\right]\right\}
\end{aligned}
$$

which contains both the zero-temperature term and the thermal correction. This result for the equilibrium force was already obtained by different authors in the framework of scatteringmatrix theory [34,35]. As remarked in [16], Eq. (77) gives a finite result for any choice of temperature and material properties for the two bodies. Moreover, the equilibrium force (77) shows the important property of depending only on the intracavity reflection operators $\mathcal{R}^{(1)+}$ and $\mathcal{R}^{(2)-}$, i.e., the operators describing the reflection produced by each body on the side of the other one.

The second term in (76) is the nonequilibrium contribution, given by

$$
\begin{aligned}
\Delta F_{1 z}\left(T_{1}, T_{2}, T_{3}\right)= & \Delta \Phi_{z}^{(\mathrm{B})}\left(T_{1}, T_{2}, T_{3}\right)-\Delta \Phi_{z}^{(\mathrm{A})}\left(T_{1}, T_{2}, T_{3}\right) \\
& +\hbar \operatorname{Tr}\left(n_{32} \mathcal{P}_{1}^{(\mathrm{pw})}\right)
\end{aligned}
$$

where the two fluxes $\Delta \Phi_{z}^{(\mathrm{B})}$ and $\Delta \Phi_{z}^{(\mathrm{A})}$ are explicitly given by Eqs. (70) and (74), respectively. The nonequilibrium contribution manifestly satisfies the condition

$$
\Delta F_{1 z}(T, T, T)=0 .
$$

Unlike the equilibrium force (77), the nonequilibrium contribution (78) still contains terms which are individually formally divergent. In Sec. VIIC, where a unified expression for the Casimir-Lifshitz force and the heat transfer will be provided, we will see that this can be manipulated so that all these divergent terms disappear.

\section{B. Heat transfer}

In order to obtain the expression for the heat transfer on body 1 we have to follow the same steps we used in the case of the force. We first need the fluxes of the Poynting vector in regions $\mathrm{B}$ and $\mathrm{A}$. Their difference will provide us the energy absorbed per unit of time by body 1 . The flux in region $B$ can be obtained by combining Eq. (30) with the correlators in region $\mathrm{B}$ given by Eq. (57). The result can be cast in the form

$$
\begin{aligned}
\varphi^{(\mathrm{B})}\left(T_{1}, T_{2}, T_{3}\right)= & \hbar \operatorname{Tr}\left\{\omega \left[n_{31} \mathcal{L}\left(\mathcal{R}^{(1)+}, \mathcal{R}^{(2)-}, \mathcal{T}^{(1)+}\right)\right.\right. \\
& +n_{23} \mathcal{L}\left(\mathcal{R}^{(2)-}, \mathcal{R}^{(1)+}, \mathcal{T}^{(2)-}\right) \\
& \left.\left.+n_{12} \mathcal{J}\left(\mathcal{R}^{(1)+}, \mathcal{R}^{(2)-}\right)\right]\right\}
\end{aligned}
$$

where

$$
\begin{aligned}
\mathcal{J}\left(\mathcal{R}^{(1)+}, \mathcal{R}^{(2)-}\right)= & U^{(12)}\left(\mathcal{P}_{-1}^{(\mathrm{pw})}-\mathcal{R}^{(1)+} \mathcal{P}_{-1}^{(\mathrm{pw})} \mathcal{R}^{(1)+\dagger}\right. \\
& \left.+\mathcal{R}^{(1)+} \mathcal{P}_{-1}^{(\mathrm{ew})}-\mathcal{P}_{-1}^{(\mathrm{ew})} \mathcal{R}^{(1)+\dagger}\right) U^{(12)^{\dagger}} \\
& \times\left(\mathcal{P}_{1}^{(\mathrm{pw})}-\mathcal{R}^{(2)-\dagger} \mathcal{P}_{1}^{(\mathrm{pw})} \mathcal{R}^{(2)-}\right. \\
& \left.+\mathcal{R}^{(2)-\dagger} \mathcal{P}_{1}^{(\mathrm{ew})}-\mathcal{P}_{1}^{(\mathrm{ew})} \mathcal{R}^{(2)-}\right), \\
\mathcal{L}\left(\mathcal{R}^{(1)+}, \mathcal{R}^{(2)-}, \mathcal{T}^{(1)+}\right)= & U^{(12)} \mathcal{T}^{(1)+} \mathcal{P}_{-1}^{\mathrm{pw}} \mathcal{T}^{(1)+\dagger} U^{(12) \dagger} \\
& \times\left(\mathcal{P}_{1}^{(\mathrm{pw})}-\mathcal{R}^{(2)-\dagger} \mathcal{P}_{1}^{(\mathrm{pw})} \mathcal{R}^{(2)-}\right. \\
& \left.+\mathcal{R}^{(2)-\dagger} \mathcal{P}_{1}^{(\mathrm{ew})}-\mathcal{P}_{1}^{(\mathrm{ew})} \mathcal{R}^{(2)-}\right) .
\end{aligned}
$$

An analogous calculation leads us to the following expression for the flux in region $\mathrm{A}$ :

$$
\begin{aligned}
\varphi^{(\mathrm{A})}( & \left.T_{1}, T_{2}, T_{3}\right) \\
= & -\hbar \operatorname{Tr}\left\{\omega \left[n_{31}\left(-\Pi^{(\mathrm{pw})}+\mathcal{P}_{1}^{(\mathrm{pw})} \mathcal{R}^{(1)-} \mathcal{P}_{-1}^{(\mathrm{pw})} \mathcal{R}^{(1)-\dagger}\right)\right.\right. \\
& +n_{32} \mathcal{P}_{1}^{(\mathrm{pw})} \mathcal{T}^{(1)-} U^{(21)} \mathcal{T}^{(2)-} \mathcal{P}_{-1}^{(\mathrm{pw})} \mathcal{T}^{(2)-\dagger} U^{(21) \dagger} \mathcal{T}^{(1)-\dagger} \\
& +n_{21}\left(\mathcal{P}_{-1}^{(\mathrm{pw})}+\mathcal{R}^{(2)-} \mathcal{P}_{-1}^{(\mathrm{ew})}-\mathcal{P}_{-1}^{(\mathrm{ew})} \mathcal{R}^{(2)-\dagger}\right. \\
& \left.-\mathcal{R}^{(2)-} \mathcal{P}_{-1}^{(\mathrm{pw})} \mathcal{R}^{(2)-\dagger}\right) U^{(21) \dagger} \mathcal{T}^{(1)-\dagger} \mathcal{P}_{1}^{(\mathrm{pw})} \mathcal{T}^{(1)-} U^{(21)} \\
& +n_{31}\left(\mathcal{P}_{1}^{(\mathrm{pw})} \mathcal{R}^{(1)-} \mathcal{P}_{-1}^{(\mathrm{pw})} \mathcal{T}^{(1)+\dagger} U^{(12) \dagger} \mathcal{R}^{(2)-\dagger} \mathcal{T}^{(1)-\dagger}\right. \\
& +\mathcal{P}_{1}^{(\mathrm{pw})} \mathcal{T}^{(1)-} \mathcal{R}^{(2)-} U^{(12)} \mathcal{T}^{(1)+} \mathcal{P}_{-1}^{(\mathrm{pw})} \mathcal{R}^{(1)-\dagger} \\
& +\mathcal{P}_{1}^{(\mathrm{pw})} \mathcal{T}^{(1)-} \mathcal{R}^{(2)-} U^{(12)} \mathcal{T}^{(1)+} \mathcal{P}_{-1}^{(\mathrm{pw})} \\
& \left.\left.\times \mathcal{T}^{(1)+\dagger} U^{(12) \dagger} \mathcal{R}^{(2)-\dagger} \mathcal{T}^{(1)-\dagger}\right)\right]
\end{aligned}
$$

The total heat flux on body 1 is finally given by the difference of the two contributions,

$$
H\left(T_{1}, T_{2}, T_{3}\right)=\varphi^{(\mathrm{A})}\left(T_{1}, T_{2}, T_{3}\right)-\varphi^{(\mathrm{B})}\left(T_{1}, T_{2}, T_{3}\right) .
$$

Since the fluxes (80) and (82) in regions B and A respectively are zero for $T_{1}=T_{2}=T_{3}$, the heat flux (83) on body 1 satisfies the evident property

$$
H(T, T, T)=0
$$

for any temperature $T \geqslant 0$.

\section{Unified expression for force and heat transfer}

We are now ready to give the main result of the paper, namely, the analytic explicit expressions for the CasimirLifshitz force and heat transfer on body 1. These expressions are valid for any choice of the shape and dielectric properties of the two bodies. We give the following definitions:

$$
\begin{gathered}
F_{1 z}\left(T_{1}, T_{2}, T_{3}\right)=\frac{F_{z}^{(\mathrm{eq})}\left(T_{1}\right)+F_{z}^{(\mathrm{eq})}\left(T_{2}\right)}{2}+\Delta_{2}\left(T_{1}, T_{2}, T_{3}\right) \\
H\left(T_{1}, T_{2}, T_{3}\right)=\Delta_{1}\left(T_{1}, T_{2}, T_{3}\right)
\end{gathered}
$$

which we can collect to give a unified expression for the nonequilibrium contribution $\Delta_{2}$ to the force and the heat transfer $\Delta_{1}$, both relative to body 1 . Before providing the explicit analytic expression of $\Delta_{m}$ for $m=1,2$, we recall that the fluxes (73) and (68) of $T_{z z}$ in regions A and B respectively contain individual divergent terms. The same property holds 
for the heat transfer, as is evident from the example of the flux (82) of $S_{z}$ in region A, containing as a first term the trace of $\Pi^{(\mathrm{pw})}$. We are going to show that the nonequilibrium force and the heat transfer are indeed convergent for any choice of the two bodies. With this aim a fundamental intermediate step is the identification of the individual divergent terms in the expressions of the fluxes of $T_{z z}$ and $S_{z}$. We first observe that all the terms which do not contain any reflection or transmission operator, such as the ones we have already discussed, are indeed divergent. This in not the case, on the contrary, for the terms proportional to at least one reflection operator, since these tend to zero in the absence of the objects. As far as the transmission operators are concerned, we have then to express each $\mathcal{T}$ operator as $1+\tilde{\mathcal{T}}$ : in analogy with the reflection operators, $\tilde{\mathcal{T}}$ tends to zero in absence of the bodies. Finally, considering the terms containing only the operators $U^{(12)}, U^{(21)}$, and projection operators, it is sufficient to use the relations (53) in order to write each of them as a sum of a divergent term, which is independent of the scattering operators, and another one proportional to the reflection matrices.

By following the procedure we have just described it can be shown that all the divergent terms exactly cancel each other. All the remaining terms are proportional to either a reflection or a modified transmission operator, as explicitly shown in Eqs. (87)-(90) below. We are now ready to give the final analytic unified expression for the Casimir-Lifshitz force and heat transfer on body 1 . This reads

$$
\begin{aligned}
\Delta_{m}\left(T_{1}, T_{2}, T_{3}\right)= & (-1)^{m+1} \hbar \operatorname{Tr}\left[\omega ^ { 2 - m } \left\{\frac { n _ { 1 2 } } { 2 } \left[\left(U^{(21) \dagger}\left(2 g_{m}\left(\mathcal{T}^{(1)-}\right)-f_{m}\left(\mathcal{R}^{(1)+}\right)\right) U^{(21)}+u_{m}\right)\left(\mathcal{P}_{-1}^{(\mathrm{pw})}+f_{-1}\left(\mathcal{R}^{(2)-}\right)\right)\right.\right.\right. \\
& \left.+(-1)^{m}\left(U^{(12)} f_{-1}\left(\mathcal{R}^{(1)+}\right) U^{(12) \dagger}+u_{-1}\right)\left(\mathcal{P}_{m}^{(\mathrm{pw})}+f_{m}\left(\mathcal{R}^{(2)-}\right)\right)\right] \\
& +n_{13}\left[-(-1)^{m}\left(U^{(12)} g_{-1}\left(\mathcal{T}^{(1)+}\right) U^{(12) \dagger}+u_{-1}\right)\left(\mathcal{P}_{m}^{(\mathrm{pw})}+f_{m}\left(\mathcal{R}^{(2)-}\right)\right)+\mathcal{P}_{m}^{(\mathrm{pw})} \mathcal{R}^{(1)-} \mathcal{P}_{-1}^{(\mathrm{pw})} \mathcal{R}^{(1)-\dagger}\right. \\
& +\left(U^{(21) \dagger} g_{m}\left(\mathcal{T}^{(1)-}\right) U^{(21)}+u_{m}\right) \mathcal{R}^{(2)-} \mathcal{P}_{-1}^{(\mathrm{pw})} \mathcal{R}^{(2)-\dagger}+U^{(21) \dagger}\left(\mathcal{P}_{m}^{(\mathrm{pw})}+g_{m}\left(\mathcal{T}^{(1)-}\right)\right) U^{(21)} \mathcal{R}^{(2)-} g_{-1}\left(\mathcal{T}^{(1)+}\right) \mathcal{R}^{(2)-\dagger} \\
& \left.+\left(\mathcal{P}_{m}^{(\mathrm{pw})} \mathcal{R}^{(1)-} \mathcal{P}_{-1}^{(\mathrm{pw})}\left(1+\tilde{\mathcal{T}}^{(1)+\dagger}\right) U^{(12) \dagger} \mathcal{R}^{(2)-\dagger}\left(1+\tilde{\mathcal{T}}^{(1)-\dagger}\right)+\text { H.c. }\right)\right] \\
& \left.\left.+n_{23} U^{(21) \dagger}\left(g_{m}\left(\mathcal{T}^{(1)-}\right)-f_{m}\left(\mathcal{R}^{(1)+}\right)\right) U^{(21)}\left(\mathcal{P}_{-1}^{(\mathrm{pw})}+g_{-1}\left(\mathcal{T}^{(2)-}\right)\right)\right\}\right]
\end{aligned}
$$

where we have defined the auxiliary functions

$$
\begin{aligned}
& f_{\alpha}(\mathcal{R})=\left\{\begin{array}{cc}
-\mathcal{R} \mathcal{P}_{-1}^{(\mathrm{pw})} \mathcal{R}^{\dagger}+\mathcal{R} \mathcal{P}_{-1}^{(\mathrm{ew})}-\mathcal{P}_{-1}^{(\mathrm{ew})} \mathcal{R}^{\dagger}, & \alpha=-1, \\
(-1)^{m} \mathcal{R}^{\dagger} \mathcal{P}_{m}^{(\mathrm{pw})} \mathcal{R}+\mathcal{R}^{\dagger} \mathcal{P}_{m}^{(\mathrm{ew})}+(-1)^{m} \mathcal{P}_{m}^{(\mathrm{ew})} \mathcal{R}, & \alpha=m \in\{1,2\},
\end{array}\right. \\
& g_{\alpha}(\mathcal{T})=\left\{\begin{array}{l}
\mathcal{T} \mathcal{P}_{-1}^{(\mathrm{pw})} \mathcal{T}^{\dagger}-\mathcal{P}_{-1}^{(\mathrm{pw})}=\tilde{\mathcal{T}} \mathcal{P}_{-1}^{(\mathrm{pw})} \tilde{\mathcal{T}}^{\dagger}+\mathcal{P}_{-1}^{(\mathrm{pw})} \tilde{\mathcal{T}}^{\dagger}+\tilde{\mathcal{T}} \mathcal{P}_{-1}^{(\mathrm{pw})}, \quad \alpha=-1, \\
\mathcal{T}^{\dagger} \mathcal{P}_{m}^{(\mathrm{pw})} \mathcal{T}-\mathcal{P}_{m}^{(\mathrm{pw})}=\tilde{\mathcal{T}}^{\dagger} \mathcal{P}_{m}^{(\mathrm{pw})} \tilde{\mathcal{T}}+\mathcal{P}_{m}^{(\mathrm{pw})} \tilde{\mathcal{T}}+\tilde{\mathcal{T}}^{\dagger} \mathcal{P}_{m}^{(\mathrm{pw})}, \quad \alpha=m \in\{1,2\}
\end{array}\right. \\
& u_{\alpha}=\left\{\begin{array}{c}
U^{(12)} \mathcal{P}_{-1}^{(\mathrm{pw})} U^{(12) \dagger}-\mathcal{P}_{-1}^{(\mathrm{pw})}=\mathcal{P}_{-1}^{(\mathrm{pw})} U^{(12) \dagger} \mathcal{R}^{(2)-\dagger} \mathcal{R}^{(1)+\dagger} \\
+\mathcal{R}^{(1)+} \mathcal{R}^{(2)-} U^{(12)} \mathcal{P}_{-1}^{(\mathrm{pw})}+\mathcal{R}^{(1)+} \mathcal{R}^{(2)-} U^{(12)} \mathcal{P}_{-1}^{(\mathrm{pw})} U^{(12) \dagger} \mathcal{R}^{(2)-\dagger} \mathcal{R}^{(1)+\dagger}, \\
U^{(21) \dagger} \mathcal{P}_{m}^{(\mathrm{pw})} U^{(21)}-\mathcal{P}_{m}^{(\mathrm{pw})}=\mathcal{P}_{m}^{(\mathrm{pw})} \mathcal{R}^{(2)-} \mathcal{R}^{(1)+} U^{(21)} \\
+U^{(21) \dagger} \mathcal{R}^{(1)+\dagger} \mathcal{R}^{(2)-\dagger} \mathcal{P}_{m}^{(\mathrm{pw})}+U^{(21) \dagger} \mathcal{R}^{(1)+\dagger} \mathcal{R}^{(2)-\dagger} \mathcal{P}_{m}^{(\mathrm{pw})} \mathcal{R}^{(2)-} \mathcal{R}^{(1)+} U^{(21)}, \quad \alpha=m \in\{1,2\}
\end{array}\right.
\end{aligned}
$$

Equations (85)-(90) allow then the explicit consideration of two bodies of arbitrary geometries and dielectric properties, in a system characterized by three possibly different temperatures $T_{1}, T_{2}$, and $T_{3}$. In order to obtain the expression of the force and heat transfer on body 2, in Eq. (87) the indexes 1 and 2 must be interchanged, as well as the indexes + and - . Moreover, in the case of the force the overall sign has to be changed. In what follows we analyze this expression for several cases.

\section{FORCE AND HEAT TRANSFER ON A BODY ALONE OUT OF THERMAL EQUILIBRIUM}

Before discussing some numerical applications of Eqs. (85) and (86) for particular choices of bodies 1 and 2, we will start by applying our formalism to a simpler configuration, providing an interesting example of the role played by the reflection and transmission operators in the calculation of the force and the heat transfer. We are now going to consider the problem of a body (called body 1 ) at temperature $T_{1}$ placed in the absence of body 2 in the same environment as before having temperature $T_{3}$. In order to obtain the force and the heat transfer in this case we can exploit the result (87) and impose $\mathcal{R}^{(2) \pm}=0, \tilde{\mathcal{T}}^{(2) \pm}=0$ as well as $T_{2}=T_{3}$. We remark that the equilibrium contribution to the force in (85) goes to zero in this limit. After straightforward manipulations, the result reads

$$
\begin{aligned}
& \Delta_{m}\left(T_{1}, T_{3}\right) \\
& =(-1)^{m+1} \hbar \operatorname{Tr}\left\{\omega ^ { 2 - m } n _ { 3 1 } \mathcal { P } _ { m } ^ { ( \mathrm { pw } ) } \left[(-1)^{m} \mathcal{R}^{(1)+} \mathcal{P}_{-1}^{(\mathrm{pw})} \mathcal{R}^{(1)+\dagger}\right.\right. \\
& \quad-\mathcal{R}^{(1)-} \mathcal{P}_{-1}^{(\mathrm{pw})} \mathcal{R}^{(1)-\dagger}+(-1)^{m}\left(\tilde{\mathcal{T}}^{(1)+} \mathcal{P}_{-1}^{(\mathrm{pw})} \tilde{\mathcal{T}}^{(1)+\dagger}\right.
\end{aligned}
$$




$$
\begin{aligned}
& \left.+\tilde{\mathcal{T}}^{(1)+} \mathcal{P}_{-1}^{(\mathrm{pw})}+\mathcal{P}_{-1}^{(\mathrm{pw})} \tilde{\mathcal{T}}^{(1)+\dagger}\right)-\left(\tilde{\mathcal{T}}^{(1)-} \mathcal{P}_{-1}^{(\mathrm{pw})} \tilde{\mathcal{T}}^{(1)-\dagger}\right. \\
& \left.\left.\left.+\tilde{\mathcal{T}}^{(1)-} \mathcal{P}_{-1}^{(\mathrm{pw})}+\mathcal{P}_{-1}^{(\mathrm{pw})} \tilde{\mathcal{T}}^{(1)-\dagger}\right)\right]\right\}
\end{aligned}
$$

Clearly, this expression is in general different from zero. Considering the particular case of thermal equilibrium $T_{1}=$ $T_{3}$, we see that $\Delta_{m}$ goes to zero: as expected, at thermal equilibrium no force is acting on a body alone independently from its geometrical properties, and it does not exchange any heat with the environment. On the contrary, if $T_{1} \neq T_{3}$, the force and the heat transfer are linked to the different behaviors of reflection and transmission on the two sides of the body. In particular, if the body is symmetric with respect to a plane $z=z_{0}$, it is easy to show that the matrix elements of reflection and transmission operators on the two sides cancel each other in the case of the force $(m=1)$. This is expected for evident reasons of symmetry. Nevertheless, even under this specific assumption, the heat transfer $[m=2$ in Eq. (91)] still remains different from zero.

\section{SOME APPLICATIONS}

In this section we are going to perform some applications of Eqs. (85) and (86). In particular, we are going to discuss the force acting on a neutral atom in front of a planar slab of finite thickness, as well as the force and the heat transfer in the case of two parallel slabs. With this aim we will provide the reflection and transmission operators associated with an atom and a planar slab.

\section{A. Force between an atom and a slab}

Let us start with the case of a neutral atom (body 2) in front of a slab (body 1) having finite thickness $\delta_{1}$. The atom has position $\mathbf{R}_{A}=\left(\mathbf{r}_{A}, z_{A}\right)=\left(0,0, z_{A}\right)$ (we have chosen $\mathbf{r}_{A}=\mathbf{0}$ by virtue of the cylindrical symmetry of the problem with respect to the axis $z=0$ ) with $z_{A}>0$, whereas the slab is defined by the two interfaces $z=0$ and $z=-\delta_{1}$, as shown in Fig. 4. This configuration is interesting since it implies the presence of a body (the atom) not characterized by translational invariance and thus for which the plane-wave basis is not a natural choice. Nevertheless, we will show that the knowledge of the atomic scattering operator in this basis, chosen in our calculation for convenience, allows us to reproduce the known results in some particular limiting cases and to give the general expression in the presence of three different temperatures $T_{1}, T_{2}$, and $T_{3}$.

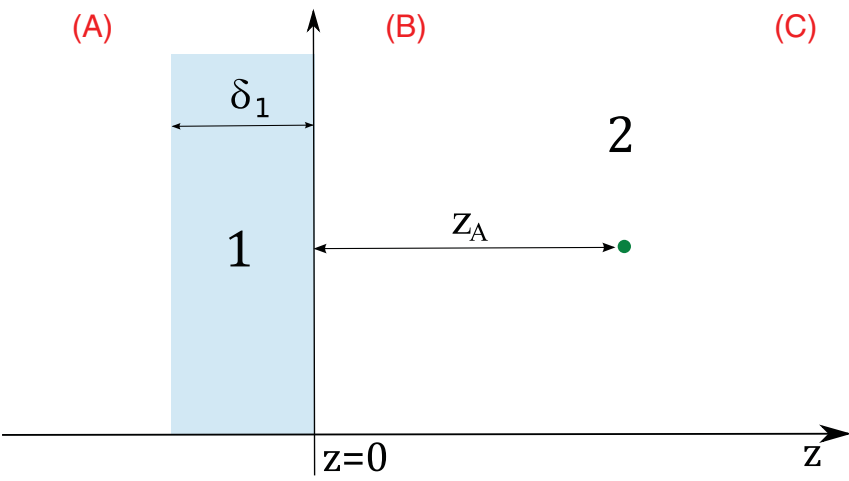

FIG. 4. (Color online) Geometry of the atom-slab configuration.
We now first discuss the reflection and transmission operators for the slab, $\mathcal{R}^{(1)+}$ and $\mathcal{T}^{(1)+}$. For homogeneous flat slabs, these operators are diagonal and given by

$$
\begin{aligned}
\left\langle p, \mathbf{k}\left|\mathcal{R}^{(1)+}\right| p^{\prime}, \mathbf{k}^{\prime}\right\rangle & =(2 \pi)^{2} \delta\left(\mathbf{k}-\mathbf{k}^{\prime}\right) \delta_{p p^{\prime}} \rho_{1 p}(\mathbf{k}, \omega), \\
\left\langle p, \mathbf{k}\left|\mathcal{T}^{(1)+}\right| p^{\prime}, \mathbf{k}^{\prime}\right\rangle & =(2 \pi)^{2} \delta\left(\mathbf{k}-\mathbf{k}^{\prime}\right) \delta_{p p^{\prime}} \tau_{1 p}(\mathbf{k}, \omega),
\end{aligned}
$$

and defined in terms of the Fresnel reflection and transmission coefficients modified by the finite thickness $\delta_{1}$,

$$
\begin{gathered}
\rho_{1 p}(\mathbf{k}, \omega)=r_{1 p}(\mathbf{k}, \omega) \frac{1-e^{2 i k_{z 1} \delta_{1}}}{1-r_{1 p}^{2}(\mathbf{k}, \omega) e^{2 i k_{z 1} \delta_{1}}}, \\
\tau_{1 p}(\mathbf{k}, \omega)=\frac{t_{1 p}(\mathbf{k}, \omega) \bar{t}_{1 p}(\mathbf{k}, \omega) e^{i k_{z 1} \delta_{1}}}{1-r_{1 p}^{2}(\mathbf{k}, \omega) e^{2 i k_{z 1} \delta_{1}}} .
\end{gathered}
$$

In these definitions we have introduced the $z$ component of the $\mathbf{K}$ vector inside medium 1 ,

$$
k_{z 1}=\sqrt{\varepsilon_{1}(\omega) \frac{\omega^{2}}{c^{2}}-\mathbf{k}^{2}},
$$

the ordinary vacuum-medium Fresnel reflection coefficients

$$
r_{1, \mathrm{TE}}=\frac{k_{z}-k_{z 1}}{k_{z}+k_{z 1}}, \quad r_{1, \mathrm{TM}}=\frac{\varepsilon_{1}(\omega) k_{z}-k_{z 1}}{\varepsilon_{1}(\omega) k_{z}+k_{z 1}},
$$

as well as both the vacuum-medium (denoted with $t$ ) and medium-vacuum (denoted with $\bar{t}$ ) transmission coefficients

$$
\begin{aligned}
t_{1, \mathrm{TE}} & =\frac{2 k_{z}}{k_{z}+k_{z 1}}, & t_{1, \mathrm{TM}} & =\frac{2 \sqrt{\varepsilon_{1}(\omega)} k_{z}}{\varepsilon_{1}(\omega) k_{z}+k_{z 1}}, \\
\bar{t}_{1, \mathrm{TE}} & =\frac{2 k_{z 1}}{k_{z}+k_{z 1}}, & \bar{t}_{1, \mathrm{TM}} & =\frac{2 \sqrt{\varepsilon_{1}(\omega)} k_{z 1}}{\varepsilon_{1}(\omega) k_{z}+k_{z 1}} .
\end{aligned}
$$

The other ingredient of our calculation is represented by the atomic scattering operators. As discussed in [38], these operators can be deduced, in dipole approximation, starting from the description of the atom as an induced dipole $\mathbf{d}(\omega)=\alpha(\omega) \mathbf{E}\left(\mathbf{R}_{A}, \omega\right)$ proportional to the component of the electric field at frequency $\omega$ calculated at the atomic position $\mathbf{R}_{A}$. The proportionality factor coincides with the atomic dynamical polarizability $\alpha(\omega)$. The field radiated by the induced dipole can thus be written analytically as a function of any incoming field; this produced field has then to be decomposed in plane waves. The expression for the outgoing amplitudes as a function of the incoming ones provides the explicit expression for the atomic reflection and transmission operators. They read (for $\phi=+,-$ )

$$
\begin{aligned}
\left\langle\mathbf{k}, p\left|\mathcal{R}_{A}^{\phi}(\omega)\right| \mathbf{k}^{\prime}, p^{\prime}\right\rangle= & \frac{i \omega^{2} \alpha(\omega)}{2 \epsilon_{0} c^{2} k_{z}}\left[\hat{\boldsymbol{\epsilon}}_{p}^{\phi}(\mathbf{k}, \omega) \cdot \hat{\boldsymbol{\epsilon}}_{p^{\prime}}^{-\phi}\left(\mathbf{k}^{\prime}, \omega\right)\right] \\
& \times \exp \left[i\left(\mathbf{k}^{\prime}-\mathbf{k}\right) \cdot \mathbf{r}_{A}\right] \\
& \times \exp \left[-i \phi\left(k_{z}+k_{z}^{\prime}\right) z_{A}\right], \\
\left\langle\mathbf{k}, p\left|\tilde{\mathcal{T}}_{A}^{\phi}(\omega)\right| \mathbf{k}^{\prime}, p^{\prime}\right\rangle= & \frac{i \omega^{2} \alpha(\omega)}{2 \epsilon_{0} c^{2} k_{z}}\left[\hat{\boldsymbol{\epsilon}}_{p}^{\phi}(\mathbf{k}, \omega) \cdot \hat{\boldsymbol{\epsilon}}_{p^{\prime}}^{\phi}\left(\mathbf{k}^{\prime}, \omega\right)\right] \\
& \times \exp \left[i\left(\mathbf{k}^{\prime}-\mathbf{k}\right) \cdot \mathbf{r}_{A}\right] \\
& \times \exp \left[-i \phi\left(k_{z}-k_{z}^{\prime}\right) z_{A}\right] .
\end{aligned}
$$

We remark that we provided the modified atomic transmission operator $\tilde{\mathcal{T}}_{A}^{\phi}$ (as a matter of fact, it clearly goes to zero in the absence of the atom) and that both operators are nondiagonal 
with respect to the wave vector $\mathbf{k}$ and the polarization $p$, as a result of the lack of translational invariance on the $x-y$ plane. Moreover, since we have attributed a temperature $T_{2}$ to the atom, the atomic polarizability $\alpha(\omega)$ must be the one associated with a thermal state at the same temperature.

We are now ready to calculate the equilibrium and nonequilibrium forces on the atom. Coherently with the dipole approximation, we have to keep only the leading-order terms in these expressions with respect to the atomic polarizability, and thus to its scattering operators (97). Moreover, the appropriate changes have to be made in Eq. (87), considering that we are in this case calculating the force on the body 2. As shown in [38], this procedure leads to the expression of the force on the atom at thermal equilibrium deduced using several different independent approaches. Focusing on the nonequilibrium contribution, after some simple algebraic manipulations, we obtain

$$
\begin{aligned}
\Delta_{2}\left(T_{1}, T_{2}, T_{3}\right) \\
=-\frac{\hbar}{4 \pi^{2} \epsilon_{0} c^{2}} \operatorname{Im}\left\{\sum_{p} \int_{0}^{+\infty} d \omega \omega^{2} \alpha(\omega)\right. \\
\times\left[n_{13} \int_{0}^{\omega / c} d k k\left(\left|\rho_{1 p}\right|^{2}+\left|\tau_{1 p}\right|^{2}-1\right)\right. \\
+\int_{0}^{\omega / c} d k k\left(\hat{\boldsymbol{\epsilon}}_{p}^{+} \cdot \hat{\boldsymbol{\epsilon}}_{p}^{-}\right)\left(n_{31} \rho_{1 p} e^{2 i k_{z} z_{A}}+n_{23} \rho_{1 p}^{*} e^{-2 i k_{z} z_{A}}\right) \\
\left.\left.+n_{21} \int_{\omega / c}^{+\infty} d k k\left(\hat{\boldsymbol{\epsilon}}_{p}^{+} \cdot \hat{\boldsymbol{\epsilon}}_{p}^{-}\right) \rho_{1 p}^{*} e^{2 i k_{z} z_{A}}\right]\right\},
\end{aligned}
$$

where the dependence on the variables $\omega$ and $k$ of all the quantities inside the integral is kept implicit. The first term in the square brackets in Eq. (98) does not depend on the atomic position $z_{A}$ and it was already identified in [9]. On the contrary, the second and the third terms do depend on the atom-slab distance $z_{A}$, but they come from different regions of the spectrum: the former results from propagative waves only, the latter from the evanescent sector. As a check of coherence with previous results, we have reobtained the expression deduced in [9] using a different approach. With this aim, we assumed that the atom occupies its ground state $\left(T_{2}=0 \mathrm{~K}\right)$ and that the slab and environmental temperatures $T_{1}$ and $T_{3}$ are such that no atomic excitation is possible: this corresponds to the replacement of the frequency-dependent dynamical polarizability $\alpha(\omega)$ with its static value $\alpha(0)$.

\section{B. Force between two slabs}

The case of two parallel homogeneous dielectric slabs of finite thickness will now be examined. This configuration, already studied in [10] in the case of infinite thickness, shows the advantage of keeping the translational symmetry, making all the scattering operators diagonal in the $(\mathbf{k}, p)$ basis, allowing at the same time a study of the effect of the environmental temperature, by virtue of the finite thickness of the slabs. Let us assume that the slab $i(i=1,2)$ has thickness $\delta_{i}$ and denote by $d$ the distance between the two slabs: in particular, slab 1 occupies the region $-\delta_{1}<z<0$ (as in the atom-slab configuration described in Sec. IX A) whereas slab 2 coincides with $d<z<d+\delta_{2}$, as shown in Fig. 5. The reflection and

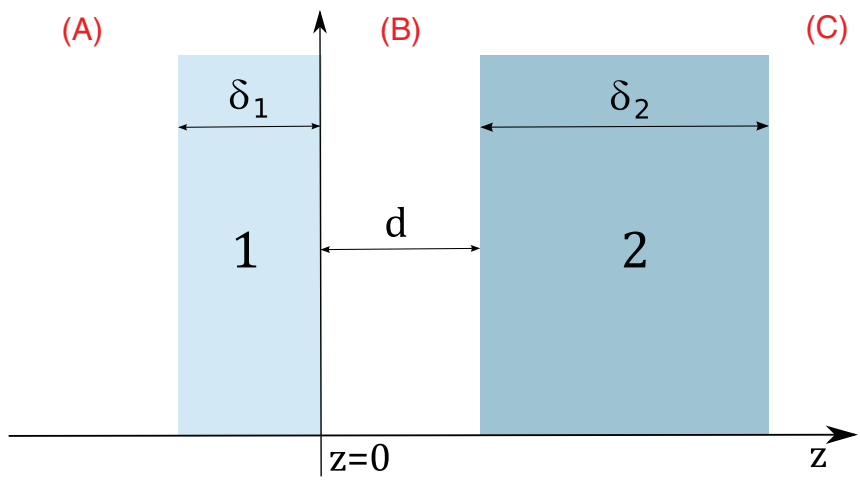

FIG. 5. (Color online) Geometry of the slab-slab configuration.

transmission operators $\mathcal{R}^{(1)+}$ and $\mathcal{T}^{(1)+}$ for slab 1 are still given by Eq. (92). The operators $\mathcal{R}^{(1)-}$ and $\mathcal{T}^{(1)-}$ associated with the left side of body 1 as well as all the scattering operators of body 2 can be obtained by solving the problem of the behavior of the scattering operators with respect to changes of frame of reference, discussed in Appendix A. The result is that the matrix elements of $\mathcal{T}^{(1)+}$ coincide with those of $\mathcal{T}^{(1)-}$ given by Eq. (92), while the interchange of 1 and 2 provides directly the elements of the transmission operator $\mathcal{T}^{(2)-}$. As far as the reflection operators are concerned we have

$$
\begin{gathered}
\left\langle p, \mathbf{k}\left|\mathcal{R}^{(1)-}\right| p^{\prime}, \mathbf{k}^{\prime}\right\rangle=(2 \pi)^{2} \delta\left(\mathbf{k}-\mathbf{k}^{\prime}\right) \delta_{p p^{\prime}} \rho_{1 p}(\mathbf{k}, \omega) e^{-2 i k_{z} \delta_{1}} \\
\left\langle p, \mathbf{k}\left|\mathcal{R}^{(2)-}\right| p^{\prime}, \mathbf{k}^{\prime}\right\rangle=(2 \pi)^{2} \delta\left(\mathbf{k}-\mathbf{k}^{\prime}\right) \delta_{p p^{\prime}} \rho_{2 p}(\mathbf{k}, \omega) e^{2 i k_{z} d}
\end{gathered}
$$

Before moving to the explicit calculation of the force, we remark that in this geometrical configuration the matrix element of any scattering operator between the states $|\mathbf{k}, p\rangle$ and $\left|\mathbf{k}^{\prime}, p^{\prime}\right\rangle$ is proportional to the Dirac delta $(2 \pi)^{2} \delta\left(\mathbf{k}-\mathbf{k}^{\prime}\right)$, as evident from Eqs. (92) and (99). This property reflects indeed the translational symmetry with respect to $x$ and $y$ characterizing this system. As a consequence, the total force (76) acting on body 1 is proportional to $(2 \pi)^{2} \delta(\mathbf{0})$ and thus formally divergent. This happens since we are calculating the total force on slab 1, which is by definition infinite, while the force density, i.e., the force per unit of surface, is a finite quantity. Nevertheless, the simple analysis of the symmetrized average of the $z z$ component of the stress tensor (17) shows us that this quantity is in this case finite and independent of $\mathbf{r}$, coherently with the translational invariance. Moreover, the result for the average value of $T_{z z}$, which means the force per unit of area, is the same we would get by using the formula (76) for the force and neglecting the divergent term $(2 \pi)^{2} \delta(\mathbf{0})$.

We are thus now ready to give the explicit expression for the pressure acting on slab 1 given by Eq. (76) after neglecting the divergent term. As for the equilibrium contribution at temperature $T$, it is given by

$$
\begin{aligned}
P_{1 z}^{(\mathrm{eq})}(T)= & -4 \operatorname{Re} \sum_{p} \int_{0}^{+\infty} \frac{d \omega}{2 \pi} \int \frac{d^{2} \mathbf{k}}{(2 \pi)^{2}} \frac{k_{z}}{\omega} \\
& \times N(\omega, T) \frac{\rho_{1 p} \rho_{2 p} e^{2 i k_{z} d}}{D_{p}},
\end{aligned}
$$


where

$$
D_{p}=1-\rho_{1 p} \rho_{2 p} e^{2 i k_{z} d}
$$

and the quantities $\rho_{1 p}, \rho_{2 p}$, and $D_{p}$ implicitly depend on $\omega$ and $\mathbf{k}$. We now turn to the nonequilibrium contribution
$\Delta F_{1 z}\left(T_{1}, T_{2}, T_{3}\right)$ appearing in Eq. (76). This gives in this case the nonequilibrium pressure

$$
\begin{aligned}
\Delta P_{1 z} & \left(T_{1}, T_{2}, T_{3}\right) \\
= & A^{(\mathrm{ew})}\left(T_{1}\right)-A^{(\mathrm{ew})}\left(T_{2}\right)+B_{1}^{(\mathrm{pw})}\left(T_{1}\right)-B_{1}^{(\mathrm{pw})}\left(T_{2}\right) \\
& +B_{2}^{(\mathrm{pw})}\left(T_{3}\right)-B_{2}^{(\mathrm{pw})}\left(T_{1}\right)+B_{3}^{(\mathrm{pw})}\left(T_{3}\right)-B_{3}^{(\mathrm{pw})}\left(T_{2}\right),
\end{aligned}
$$

where we have defined

$$
\begin{aligned}
& A^{(\mathrm{ew})}(T)=\frac{\hbar}{2 \pi^{2}} \sum_{p} \int_{0}^{+\infty} d \omega \int_{\frac{\omega}{c}}^{+\infty} d k k \operatorname{Im} k_{z} n(\omega, T) \frac{\operatorname{Im}\left(\rho_{1 p} \rho_{2 p}^{*}\right)}{\left|D_{p}\right|^{2}} e^{-2 d \operatorname{Im} k_{z}}, \\
& B_{1}^{(\mathrm{pw})}(T)=-\frac{\hbar}{4 \pi^{2}} \sum_{p} \int_{0}^{+\infty} d \omega \int_{0}^{\omega / c} d k k k_{z} n(\omega, T) \frac{\left|\rho_{2 p}\right|^{2}-\left|\rho_{1 p}\right|^{2}+\left|\tau_{1 p}\right|^{2}\left(1-\left|\rho_{2 p}\right|^{2}\right)}{\left|D_{p}\right|^{2}}, \\
& B_{2}^{(\mathrm{pw})}(T)=-\frac{\hbar}{4 \pi^{2}} \sum_{p} \int_{0}^{+\infty} d \omega \int_{0}^{\omega / c} d k k k_{z} n(\omega, T)\left[\frac{\left|\tau_{1 p}\right|^{2}\left[1+\left|\rho_{2 p}\right|^{2}\left(1-\left|\tau_{1 p}\right|^{2}\right)\right]}{\left|D_{p}\right|^{2}}-\left|\rho_{1 p}\right|^{2}-2 \operatorname{Re}\left(\frac{\rho_{1 p}^{*} \rho_{2 p} \tau_{1 p}^{2}}{D_{p}} e^{2 i k_{z}\left(d+\delta_{1}\right)}\right)\right], \\
& B_{3}^{(\mathrm{pw})}(T)=-\frac{\hbar}{4 \pi^{2}} \sum_{p} \int_{0}^{+\infty} d \omega \int_{0}^{\omega / c} d k k k_{z} n(\omega, T)\left[\frac{\left|\tau_{2 p}\right|^{2}}{\left|D_{p}\right|^{2}}\left(1+\left|\rho_{1 p}\right|^{2}-\left|\tau_{1 p}\right|^{2}\right)-1\right] .
\end{aligned}
$$

We note that the last term of the last line can be explicitly integrated. It gives to the nonequilibrium force (102) a contribution $2 \sigma\left(T_{3}^{4}-T_{2}^{4}\right) / 3 c$ where $\sigma=\pi^{2} k_{B}^{4} / 60 c^{2} \hbar^{3}$ : this term is the well-known Stefan-Boltzmann radiation pressure. We have verified that in the limit of infinite thickness, corresponding to $\tau_{1 p}, \tau_{2 p} \rightarrow 0$ and the replacement of $\rho_{1 p}$ and $\rho_{2 p}$ with the ordinary Fresnel coefficients, we analytically reobtain the results already deduced in $[10,13]$.

We then numerically evaluated, using Eqs. (100) and (102) arranged as in Eq. (85), the total pressure acting on a $2-\mu \mathrm{m}-$ thick slab 1 , made of fused silica, in front of a $1000-\mu \mathrm{m}$-thick slab 2, made of silicon. The optical data for the two materials are taken from [39]. We have considered different sets of temperatures $\left(T_{1}, T_{2}, T_{3}\right)$. The results are shown in Figs. 6 and 7.

In Fig. 6 a wide range of distances, from 1 to $10 \mu \mathrm{m}$, has been considered, for different equilibrium and nonequilibrium thermal configurations. In particular, Figs. 6(a), 6(b), and 6(c) correspond to three different choices of the slab temperatures $T_{1}$ and $T_{2}$ (see caption of Fig. 6 for details). For each case we have represented the nonequilibrium pressure corresponding to the values of the environmental temperature $T_{3}=0,300,600$ $\mathrm{K}$, as well as the equilibrium pressure at the same three temperatures. We note that the transition from an equilibrium to a nonequilibrium configuration can dramatically change both the qualitative and quantitative behavior of the interaction. It is worth noting that, even for fixed values of $T_{1}, T_{2}$, and the slab-slab distance $d$, the value of $T_{3}$ may significantly affect the value of the pressure, even by orders of magnitude. As a consequence, the environmental temperature can, remarkably, be considered as an efficient tool to tune the interaction. This feature is equally present in the case described in Fig. 6(c), where $T_{1}$ equals $T_{2}$. This underlines that even in experiments devoted to the measure of the force at thermal equilibrium the environmental temperature should be carefully controlled.
All these effects prove to be even more spectacular when we look at Fig. 7, where linear scales are employed. Indeed, in the case of $T_{3}=0 \mathrm{~K}$ the pressure becomes exactly zero at a given distance around $6 \mu \mathrm{m}$, and repulsive for larger distances.

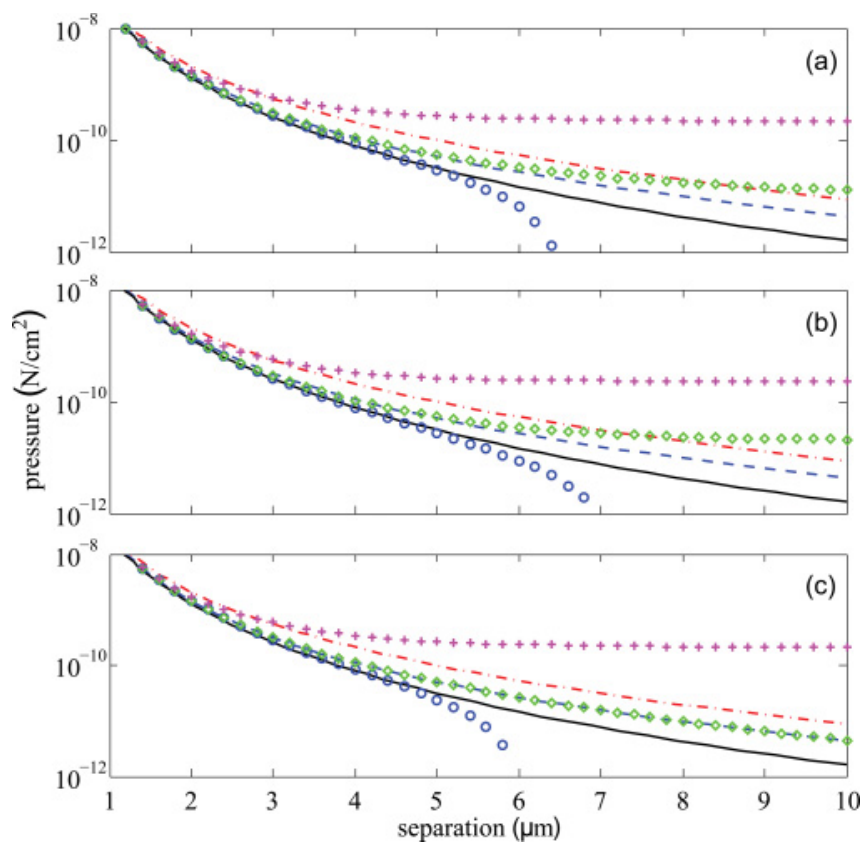

FIG. 6. (Color online) Slab-slab geometry (see Sec. IX B). Pressure (102) acting on a $\delta_{1}=2-\mu \mathrm{m}$-thick slab (body 1 , fused silica) parallel to a $\delta_{2}=1000-\mu$ m-thick slab (body 2, silicon). Lines: equilibrium pressures at $T=0 \mathrm{~K}$ (black solid), $300 \mathrm{~K}$ (blue dashed), and $600 \mathrm{~K}$ (red dash-dotted). Symbols: nonequilibrium pressures at $T_{3}=0 \mathrm{~K}$ (blue circles), $300 \mathrm{~K}$ (green diamonds), and $600 \mathrm{~K}$ (magenta plus). $T_{1}=300 \mathrm{~K}$ and $T_{2}=0 \mathrm{~K}$ in (a), $T_{1}=0 \mathrm{~K}$ and $T_{2}=300 \mathrm{~K}$ in (b), and $T_{1}=T_{2}=300 \mathrm{~K}$ in (c). 
The appearance of repulsive interactions with nonequilibrium systems has been previously shown only for microscopic bodies, and in particular for the atom-surface interaction [11]. Moreover, the possibility of drastically reducing the CasimirLifshitz force may be useful in investigations of hypothetical smaller forces of different origins [40-44].

\section{Heat transfer between two slabs}

In analogy with the force, we have chosen the same slab-slab configuration to provide a numerical application of Eq. (86) giving the heat transfer on body 1 for any choice of $T_{1}, T_{2}$, and $T_{3}$. This case was already studied in [25], where the influence of the environmental temperature $T_{3}$ was not considered. Also in this case, the result is expressed per unit of surface: we then obtain the energy $h_{1}$ absorbed per unit of surface and per unit of time by the slab 1 . Its analytic expression, using the same formalism of Sec. IX B, reads

$$
\begin{aligned}
h_{1}\left(T_{1}, T_{2}, T_{3}\right)= & \mathcal{A}^{(\mathrm{ew})}\left(T_{1}\right)-\mathcal{A}^{(\mathrm{ew})}\left(T_{2}\right) \\
& +\mathcal{B}_{1}^{(\mathrm{pw})}\left(T_{1}\right)-\mathcal{B}_{1}^{(\mathrm{pw})}\left(T_{2}\right) \\
& +\mathcal{B}_{2}^{(\mathrm{pw})}\left(T_{3}\right)-\mathcal{B}_{2}^{(\mathrm{pw})}\left(T_{1}\right) \\
& +\mathcal{B}_{3}^{(\mathrm{pw})}\left(T_{3}\right)-\mathcal{B}_{3}^{(\mathrm{pw})}\left(T_{2}\right),
\end{aligned}
$$

where we have defined

$$
\begin{gathered}
\mathcal{A}^{(\mathrm{ew})}(T)=\frac{\hbar}{2 \pi^{2}} \sum_{p} \int_{0}^{+\infty} d \omega \int_{\omega / c}^{+\infty} d k k \omega n(\omega, T) \frac{e^{-2 d \operatorname{Im} k_{z}}}{\left|D_{p}\right|^{2}}\left[\operatorname{Re}\left(\rho_{1 p} \rho_{2 p}\right)-\operatorname{Re}\left(\rho_{1 p} \rho_{2 p}^{*}\right)\right], \\
\mathcal{B}_{1}^{(\mathrm{pw})}(T)=\frac{\hbar}{4 \pi^{2}} \sum_{p} \int_{0}^{+\infty} d \omega \int_{0}^{\omega / c} d k k \omega n(\omega, T) \frac{\left|\rho_{1 p}\right|^{2}+\left|\rho_{2 p}\right|^{2}-1-\left|\rho_{1 p} \rho_{2 p}\right|^{2}+\left|\tau_{1 p}\right|^{2}\left(1-\left|\rho_{2 p}\right|^{2}\right)}{\left|D_{p}\right|^{2}} \\
\mathcal{B}_{2}^{(\mathrm{pw})}(T)=\frac{\hbar}{4 \pi^{2}} \sum_{p} \int_{0}^{+\infty} d \omega \int_{0}^{\omega / c} d k k \omega n(\omega, T)\left[1-\left|\rho_{1 p}\right|^{2}-\frac{\left|\tau_{1 p}\right|^{2}\left[1-\left|\rho_{2 p}\right|^{2}\left(1-\left|\tau_{1 p}\right|^{2}\right)\right]}{\left|D_{p}\right|^{2}}-2 \operatorname{Re}\left(\frac{\rho_{1 p}^{*} \rho_{2 p} \tau_{1 p}^{2}}{D_{p}} e^{2 i k_{z}\left(d+\delta_{1}\right)}\right)\right], \\
\mathcal{B}_{3}^{(\mathrm{pw})}(T)=\frac{\hbar}{4 \pi^{2}} \sum_{p} \int_{0}^{+\infty} d \omega \int_{0}^{\omega / c} d k k \omega n(\omega, T) \frac{\left|\tau_{2 p}\right|^{2}}{\left|D_{p}\right|^{2}}\left(1-\left|\rho_{1 p}\right|^{2}-\left|\tau_{1 p}\right|^{2}\right) .
\end{gathered}
$$

We have numerically evaluated the heat transfer (104) on body 1 for different set of temperatures $\left(T_{1}, T_{2}, T_{3}\right)$. In particular, the cases of Figs. 8(a), 8(b), and 8(c) correspond to three different choices of the slab temperatures $T_{1}$ and $T_{2}$ (see caption for details). For each case we have represented the heat transfer corresponding to the values of the environmental temperature $T_{3}=0,300,400,500,600 \mathrm{~K}$. As in the case of pressure, the role of the environmental radiation for heat transfer is particularly interesting. In Fig. 8 the heat transfer $h_{1}$ shows an oscillating behavior with an amplitude increasing with the temperature $T_{3}$. As far as the positions of minima and maxima are concerned, they are almost insensitive to the slab thicknesses $\delta_{1}$ and $\delta_{2}$ and to the three temperatures $T_{1}, T_{2}$, and $T_{3}$, being on the contrary connected to the dielectric properties of the two bodies. Furthermore, these oscillations originate from the propagative sector, as evident from the analysis of case Fig. 8(c): as a matter of fact, in this configuration, where $T_{1}$ and $T_{2}$ coincide, Eq. (104) contains only contributions of pure propagative nature $\left(B_{2}\right.$ and $\left.B_{3}\right)$. These oscillations were already theoretically studied in [1]. Another interesting property emerging from Fig. 8(a) is the occurrence of a change of sign in the heat transfer. Focusing on the blue crosses, corresponding to $\left(T_{1}, T_{2}, T_{3}\right)=(300,0,400) \mathrm{K}$, we observe that at large separations, where the propagative waves play a dominant role, the heat transfer is positive, i.e., the slab 1 absorbs energy. At smaller separations, and in particular for distances of the order of $1 \mu \mathrm{m}$ the heat transfer changes sign, becoming negative (i.e., the slab 1 radiates energy). This can be understood in terms of the evanescent-wave coupling between body 1 and body 2, which is at zero temperature. We also note that the higher is the value of $T_{3}$, the smaller is the distance at which the change of sign occurs. From the figure, we deduce

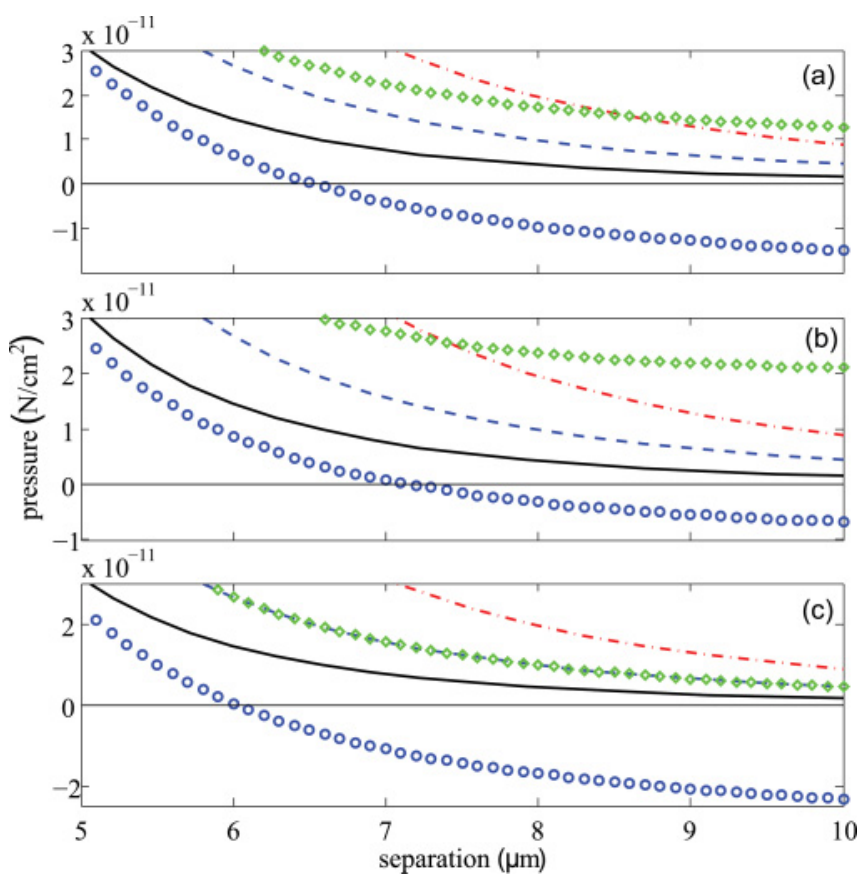

FIG. 7. (Color online) Zoom of Fig. 6 in linear scales, with the same conventions. Here the change of sign of the force clearly appears. 


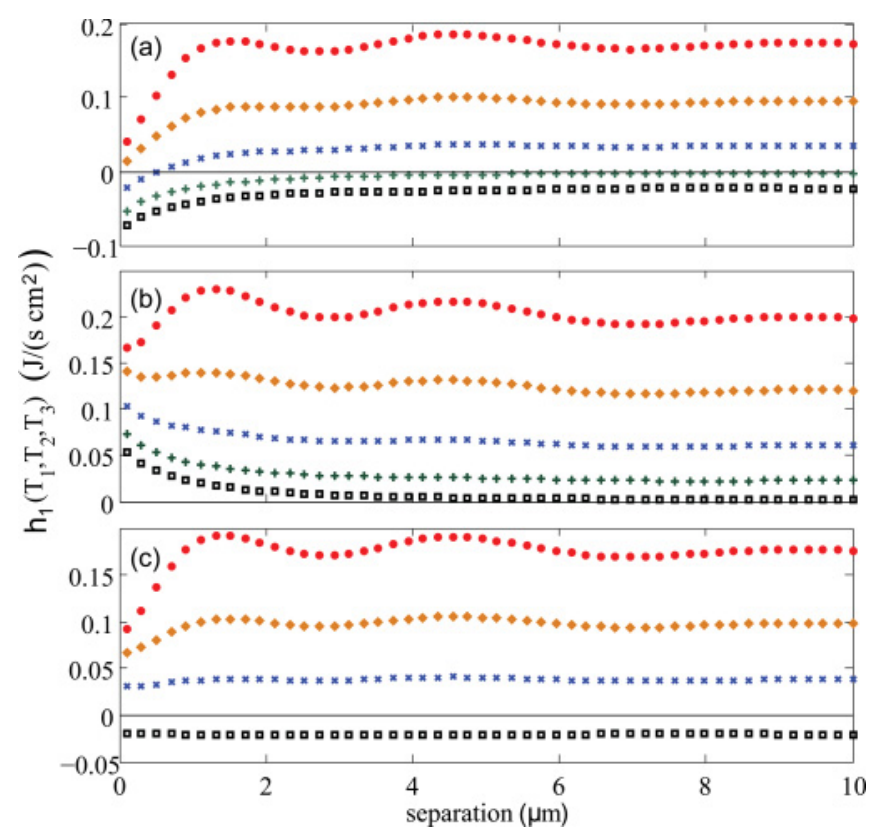

FIG. 8. (Color online) Slab-slab geometry (see Sec. IX C). Radiative heat transfer per unit of surface (104) on a $\delta_{1}=2-\mu \mathrm{m}$-thick slab (body 1 , fused silica) parallel to a $\delta_{2}=1000-\mu \mathrm{m}$-thick slab (body 2 , silicon). The temperatures of the slabs are $T_{1}=300 \mathrm{~K}$ and $T_{2}=0$ $\mathrm{K}$ in (a), $T_{1}=0 \mathrm{~K}$ and $T_{2}=300 \mathrm{~K}$ in (b), and $T_{1}=T_{2}=300 \mathrm{~K}$ in (c). Symbols: $T_{3}=0 \mathrm{~K}$ (black squares), $300 \mathrm{~K}$ (green plus), $400 \mathrm{~K}$ (blue crosses), $500 \mathrm{~K}$ (brown diamonds), and $600 \mathrm{~K}$ (red circles).

that for $T_{3}=500,600 \mathrm{~K}$ this transition happens at distances below $0.5 \mu \mathrm{m}$.

\section{CONCLUSIONS}

We presented a systematic derivation of the radiative heat transfer and the Casimir-Lifshitz force between two arbitrary bodies. We first expressed the correlating functions of the electromagnetic field in any region of the system as a function of the scattering operators of each body. This result was used to provide closed-form unified analytic expression of the heat transfer and the force. This expression fully takes into account the interaction between bodies of finite size, any shape, and any temperature, as well as the presence of a thermal external radiation coming from the environment.

We applied this theory to two simple but instructive examples: an atom in front of a slab and two parallel slabs. The former configuration is an example of a non-translationallyinvariant system, and generalizes previous results out of thermal equilibrium. The latter represents the simplest geometrical configuration in which the effects of finite size and external temperature can be quantitatively analyzed. As far as the force is concerned, we observed that the environmental temperature can substantially tune the interaction, eventually producing a repulsive force. As for the heat transfer, it shows an oscillatory behavior with respect to distance that is connected to the dielectric properties of the two slabs and whose amplitude increases with the temperature. Moreover, we found that some given choices of the environmental temperature are able to produce a heat flux whose sign changes as a function of distance.
This study shows the interest of nonequilibrium configurations, mainly consisting in a strong tunability of force and heat transfer. It would thus be interesting to apply our results to other geometrical configurations and to test them experimentally.

\section{ACKNOWLEDGMENTS}

The authors thank D. Felbacq, B. Guizal, and E. Rousseau for fruitful and stimulating discussions.

\section{APPDNDIX A: TRANSFORMATIONS OF SCATTERING OPERATORS UNDER TRANSLATIONS}

We will discuss here the problem of the transformation of the scattering (both reflection and transmission) operators with respect to translations. Let us suppose we have a first frame of reference $\mathcal{O}$ and a second one $\tilde{\mathcal{O}}$ obtained from the first one by a translation of $\mathbf{R}_{S}$. For the purpose of this section it is useful to gather the reflection and transmission operators $\mathcal{R}^{ \pm}$and $\mathcal{T}^{ \pm}$in a single operator $\mathcal{S}^{\phi \phi^{\prime}}$ connecting the outgoing modes propagating in direction $\phi$ to the incoming modes propagating in direction $\phi^{\prime}$. As a consequence, we have the following identification:

$$
\begin{aligned}
\mathcal{S}^{++} & =\mathcal{T}^{+}, & \mathcal{S}^{+-} & =\mathcal{R}^{+}, \\
\mathcal{S}^{-+} & =\mathcal{R}^{-}, & \mathcal{S}^{--} & =\mathcal{T}^{-} .
\end{aligned}
$$

From the definition of the electric field in $\mathcal{O}$,

$$
\begin{aligned}
\mathbf{E}(\mathbf{R}, \omega)= & \sum_{\phi, p} \int \frac{d^{2} \mathbf{k}}{(2 \pi)^{2}} \exp \left(i \mathbf{K}^{\phi} \cdot \mathbf{R}\right) \hat{\boldsymbol{\epsilon}}_{p}^{\phi}(\mathbf{k}, \omega) E_{p}^{\phi}(\mathbf{k}, \omega) \\
= & \sum_{\phi, p} \int \frac{d^{2} \mathbf{k}}{(2 \pi)^{2}} \exp \left[i \mathbf{K}^{\phi} \cdot\left(\mathbf{R}-\mathbf{R}_{S}\right)\right] \hat{\boldsymbol{\epsilon}}_{p}^{\phi}(\mathbf{k}, \omega) \\
& \times \exp \left(i \mathbf{K}^{\phi} \cdot \mathbf{R}_{S}\right) E_{p}^{\phi}(\mathbf{k}, \omega),
\end{aligned}
$$

we deduce that the amplitude $\tilde{E}_{p}^{\phi}(\mathbf{k}, \omega)$ in the new frame of reference $\tilde{\mathcal{O}}$ equals

$$
\tilde{E}_{p}^{\phi}(\mathbf{k}, \omega)=\exp \left(i \mathbf{K}^{\phi} \cdot \mathbf{R}_{S}\right) E_{p}^{\phi}(\mathbf{k}, \omega)
$$

From these properties we deduce

$$
\begin{aligned}
\tilde{E}_{p}^{(\mathrm{out}) \phi}(\mathbf{k}, \omega)= & \exp \left(i \mathbf{K}^{\phi} \cdot \mathbf{R}_{S}\right) E_{p}^{(\mathrm{out}) \phi}(\mathbf{k}, \omega) \\
= & \exp \left(i \mathbf{K}^{\phi} \cdot \mathbf{R}_{S}\right) \sum_{p^{\prime}} \int \frac{d^{2} \mathbf{k}^{\prime}}{(2 \pi)^{2}} \\
& \times\left\langle p, \mathbf{k}\left|\mathcal{S}^{\phi \phi^{\prime}}\right| p^{\prime}, \mathbf{k}^{\prime}\right\rangle E_{p^{\prime}}^{(\mathrm{in}) \phi^{\prime}}\left(\mathbf{k}^{\prime}, \omega\right) \\
= & \exp \left(i \mathbf{K}^{\phi} \cdot \mathbf{R}_{S}\right) \sum_{p^{\prime}} \int \frac{d^{2} \mathbf{k}^{\prime}}{(2 \pi)^{2}}\left\langle p, \mathbf{k}\left|\mathcal{S}^{\phi \phi^{\prime}}\right| p^{\prime}, \mathbf{k}^{\prime}\right\rangle \\
& \times \exp \left(-i \mathbf{K}^{\prime \phi^{\prime}} \cdot \mathbf{R}_{S}\right) \tilde{E}_{p^{\prime}}^{(\mathrm{in}) \phi^{\prime}}\left(\mathbf{k}^{\prime}, \omega\right),
\end{aligned}
$$

and finally the link between the matrix element of the scattering operators in the two frames of reference,

$$
\begin{aligned}
\left\langle p, \mathbf{k}\left|\tilde{\mathcal{S}}^{\phi \phi^{\prime}}\right| p^{\prime}, \mathbf{k}^{\prime}\right\rangle= & \left.\exp \left[i\left(\mathbf{K}^{\phi}-\mathbf{K}^{\prime} \phi^{\prime}\right) \cdot \mathbf{R}_{S}\right)\right] \\
& \times\left\langle p, \mathbf{k}\left|\mathcal{S}^{\phi \phi^{\prime}}\right| p^{\prime}, \mathbf{k}^{\prime}\right\rangle .
\end{aligned}
$$


In the particular and important case of translation along the $z$ axis, assuming that the origin of $\tilde{\mathcal{O}}$ has coordinates $(0,0, d)$ with respect to $\mathcal{O}$, we have

$$
\begin{gathered}
\left\langle p, \mathbf{k}\left|\tilde{\mathcal{R}}^{+}\right| p^{\prime}, \mathbf{k}^{\prime}\right\rangle=\exp \left[i\left(k_{z}+k_{z}^{\prime}\right) d\right]\left\langle p, \mathbf{k}\left|\mathcal{R}^{+}\right| p^{\prime}, \mathbf{k}^{\prime}\right\rangle, \\
\left\langle p, \mathbf{k}\left|\tilde{\mathcal{R}}^{-}\right| p^{\prime}, \mathbf{k}^{\prime}\right\rangle=\exp \left[-i\left(k_{z}+k_{z}^{\prime}\right) d\right]\left\langle p, \mathbf{k}\left|\mathcal{R}^{-}\right| p^{\prime}, \mathbf{k}^{\prime}\right\rangle, \\
\left\langle p, \mathbf{k}\left|\tilde{\mathcal{T}}^{+}\right| p^{\prime}, \mathbf{k}^{\prime}\right\rangle=\exp \left[i\left(k_{z}-k_{z}^{\prime}\right) d\right]\left\langle p, \mathbf{k}\left|\mathcal{T}^{+}\right| p^{\prime}, \mathbf{k}^{\prime}\right\rangle, \\
\left\langle p, \mathbf{k}\left|\tilde{\mathcal{T}}^{-}\right| p^{\prime}, \mathbf{k}^{\prime}\right\rangle=\exp \left[-i\left(k_{z}-k_{z}^{\prime}\right) d\right]\left\langle p, \mathbf{k}\left|\mathcal{T}^{-}\right| p^{\prime}, \mathbf{k}^{\prime}\right\rangle .
\end{gathered}
$$

Apart from their general theoretical interest, these relations are used in Sec. IX in order to deduce any reflection and transmission operator associated with a planar slab as a function of the ordinary Fresnel coefficient (modified to take into account the finite thickness), usually calculated assuming that the interface coincides with the surface $z=0$.

\section{APPDNDIX B: RECIPROCITY RELATIONS OF SCATTERING OPERATORS}

The matrix elements of the scattering operators are not all mutually independent. By exploiting some properties of the electromagnetic field, it is in fact possible to deduce some relations connecting these elements. This is the case, for example, of the reciprocity relations presented in [45]. In this appendix, we derive and express these relations using our field decomposition and notation. In [45], the authors start their derivation of the reciprocity relations from Lorentz's reciprocity theorem in the presence of sources. To formulate this theorem, we start by supposing that we have, in the presence of a given body, a dipole $\mathbf{p}_{1}\left(\mathbf{p}_{2}\right)$ in position $\mathbf{R}_{1}\left(\mathbf{R}_{2}\right)$ and oscillating at frequency $\omega$. Each of these dipoles produces an electromagnetic field which is then scattered by the body and reaches the other dipole. Lorentz's reciprocity theorem can then be formulated by imposing the requirement that

$$
\mathbf{p}_{1} \cdot \mathbf{E}_{2}\left(\mathbf{R}_{1}\right)=\mathbf{p}_{2} \cdot \mathbf{E}_{1}\left(\mathbf{R}_{2}\right)
$$

where $\mathbf{E}_{1}\left(\mathbf{R}_{2}\right)\left[\mathbf{E}_{2}\left(\mathbf{R}_{1}\right)\right]$ is the result of the scattering on the body of the field produced by the dipole $\mathbf{p}_{1}\left(\mathbf{p}_{2}\right)$ and then calculated at the position of dipole $\mathbf{p}_{2}\left(\mathbf{p}_{1}\right)$.

In order to fix the notation, let us suppose that the dipole $\mathbf{p}_{1}$ is on the side $\phi$ of the body, while $\mathbf{p}_{2}$ is on the side $\phi^{\prime}$. In order to calculate the field $\mathbf{E}_{1}\left(\mathbf{R}_{2}\right)$, we need to take the component of the field emitted by $\mathbf{p}_{1}$ propagating in direction $-\phi$, then apply the operator $\mathcal{S}^{\phi^{\prime},-\phi}$, and calculate the resulting field in position $\mathbf{R}_{2}$. The component at the frequency $\omega$ of the field produced by a dipole $\mathbf{p}$ in position $\mathbf{R}_{p}$ reads

$$
\mathbf{E}(\mathbf{R}, \omega)=\frac{1}{4 \pi \epsilon_{0}} \nabla_{\mathbf{R}} \times \nabla_{\mathbf{R}} \times\left[\mathbf{p} \frac{e^{i(\omega / c) R_{d}}}{R_{d}}\right],
$$

where $R_{d}=\left|\mathbf{R}_{d}\right|=\left|\mathbf{R}-\mathbf{R}_{p}\right|$ and $\nabla_{\mathbf{R}}$ represents the gradient with respect to $\mathbf{R}$. As discussed in [38], the passage from the spherical wave emitted by the dipole and our angular spectrum representation can be performed by using the Weyl representation [46]; the resulting field propagating in the $\phi$ direction from position $\mathbf{R}_{p}$ reads, after straightforward algebraic manipulations,

$$
\begin{aligned}
\mathbf{E}^{\phi}(\mathbf{R}, \omega)= & \frac{i \omega^{2}}{2 \epsilon_{0} c^{2}} \sum_{p} \int \frac{d^{2} \mathbf{k}}{(2 \pi)^{2}} \frac{1}{k_{z}} \hat{\boldsymbol{\epsilon}}_{p}^{\phi}(\mathbf{k}, \omega) \\
& \times\left[\hat{\boldsymbol{\epsilon}}_{p}^{\phi}(\mathbf{k}, \omega) \cdot \mathbf{p}\right] \exp \left[i \mathbf{K}^{\phi} \cdot\left(\mathbf{R}-\mathbf{R}_{p}\right)\right] .
\end{aligned}
$$

We can then simply deduce the expression

$$
\begin{aligned}
\mathbf{E}_{1}\left(\mathbf{R}_{2}\right)= & \frac{i \omega^{2}}{2 \epsilon_{0} c^{2}} \sum_{p p^{\prime}} \int \frac{d^{2} \mathbf{k}}{(2 \pi)^{2}} \int \frac{d^{2} \mathbf{k}^{\prime}}{(2 \pi)^{2}} \hat{\boldsymbol{\epsilon}}_{p}^{\phi^{\prime}}(\mathbf{k}, \omega) \\
& \times \frac{1}{k_{z}^{\prime}}\left[\mathbf{p}_{1} \cdot \hat{\boldsymbol{\epsilon}}_{p^{\prime}}^{-\phi}\left(\mathbf{k}^{\prime}, \omega\right)\right]\left\langle\mathbf{k}, p\left|\mathcal{S}^{\phi^{\prime},-\phi}\right| \mathbf{k}^{\prime}, p^{\prime}\right\rangle \\
& \times \exp \left[i\left(\mathbf{K}^{\phi^{\prime}} \cdot \mathbf{R}_{2}-\mathbf{K}^{\prime}-\phi \cdot \mathbf{R}_{1}\right)\right],
\end{aligned}
$$

and analogously

$$
\begin{aligned}
\mathbf{E}_{2}\left(\mathbf{R}_{1}\right)= & \frac{i \omega^{2}}{2 \epsilon_{0} c^{2}} \sum_{p p^{\prime}} \int \frac{d^{2} \mathbf{k}}{(2 \pi)^{2}} \int \frac{d^{2} \mathbf{k}^{\prime}}{(2 \pi)^{2}} \hat{\boldsymbol{\epsilon}}_{p^{\prime}}^{\phi}\left(-\mathbf{k}^{\prime}, \omega\right) \\
& \times \frac{1}{k_{z}}\left[\mathbf{p}_{2} \cdot \hat{\boldsymbol{\epsilon}}_{p}^{-\phi^{\prime}}(-\mathbf{k}, \omega)\right]\left\langle-\mathbf{k}^{\prime}, p^{\prime}\left|\mathcal{S}^{\phi,-\phi^{\prime}}\right|-\mathbf{k}, p\right\rangle \\
& \times \exp \left[i\left(\mathbf{K}^{\phi^{\prime}} \cdot \mathbf{R}_{2}-\mathbf{K}^{\prime}-\phi \cdot \mathbf{R}_{1}\right)\right]
\end{aligned}
$$

where we have performed the change of variables

$$
\left(\mathbf{k}, \mathbf{k}^{\prime}, p, p^{\prime}\right) \longrightarrow\left(-\mathbf{k}^{\prime},-\mathbf{k}, p^{\prime}, p\right) .
$$

By imposing the condition (B1) we get

$$
\begin{aligned}
k_{z}\left[\mathbf{p}_{1} \cdot \hat{\boldsymbol{\epsilon}}_{p^{\prime}}^{-\phi}\left(\mathbf{k}^{\prime}, \omega\right)\right]\left[\mathbf{p}_{2} \cdot \hat{\boldsymbol{\epsilon}}_{p}^{\phi^{\prime}}(\mathbf{k}, \omega)\right]\left\langle\mathbf{k}, p\left|\mathcal{S}^{\phi^{\prime},-\phi}\right| \mathbf{k}^{\prime}, p^{\prime}\right\rangle \\
=k_{z}^{\prime}\left[\mathbf{p}_{1} \cdot \hat{\boldsymbol{\epsilon}}_{p^{\prime}}^{\phi}\left(-\mathbf{k}^{\prime}, \omega\right)\right]\left[\mathbf{p}_{2} \cdot \hat{\boldsymbol{\epsilon}}_{p}^{-\phi^{\prime}}(-\mathbf{k}, \omega)\right] \\
\quad \times\left\langle-\mathbf{k}^{\prime}, p^{\prime}\left|\mathcal{S}^{\phi,-\phi^{\prime}}\right|-\mathbf{k}, p\right\rangle,
\end{aligned}
$$

from which we deduce, by using the properties of the polarization unit vectors $(8)$, the final relation

$$
k_{z}\left\langle\mathbf{k}, p\left|\mathcal{S}^{\phi^{\prime},-\phi}\right| \mathbf{k}^{\prime}, p^{\prime}\right\rangle=k_{z}^{\prime}(-1)^{p+p^{\prime}}\left\langle-\mathbf{k}^{\prime}, p^{\prime}\left|\mathcal{S}^{\phi,-\phi^{\prime}}\right|-\mathbf{k}, p\right\rangle .
$$

By choosing the four possible values of the couple $\left(\phi, \phi^{\prime}\right)$, we obtain the relations

$$
\begin{aligned}
& k_{z}\left\langle\mathbf{k}, p\left|\mathcal{T}^{ \pm}\right| \mathbf{k}^{\prime}, p^{\prime}\right\rangle=k_{z}^{\prime}(-1)^{p+p^{\prime}}\left\langle-\mathbf{k}^{\prime}, p^{\prime}\left|\mathcal{T}^{\mp}\right|-\mathbf{k}, p\right\rangle, \\
& k_{z}\left\langle\mathbf{k}, p\left|\mathcal{R}^{ \pm}\right| \mathbf{k}^{\prime}, p^{\prime}\right\rangle=k_{z}^{\prime}(-1)^{p+p^{\prime}}\left\langle-\mathbf{k}^{\prime}, p^{\prime}\left|\mathcal{R}^{ \pm}\right|-\mathbf{k}, p\right\rangle .
\end{aligned}
$$

These equations clearly show that the matrix elements of each transmission operator are connected to elements of the specular operator, while the matrix elements of each reflection operator are not independent. The relations (B9) contribute to the derivation presented in Appendix D.

\section{APPDNDIX C: GREEN FUNCTION AND SCATTERING OPERATORS}

In this appendix we are going to derive the relation between the Green function in the presence of a single body and its reflection and transmission operators. This feature is a main point in the calculation given in Appendix D. In order to derive this connection we will start from the definition of the 
Green function. Suppose we have a dipole electric moment $\mathbf{p}$ located at $\mathbf{R}^{\prime}$ oscillating at frequency $\omega$ and thus producing an electric field oscillating at the same frequency proportional to the components of the dipole moment itself. The component $G_{i j}\left(\mathbf{R}, \mathbf{R}^{\prime}\right)$ at frequency $\omega$ of the Green function can be interpreted as the part of component $i$ of the total electric field at the point $\mathbf{R}$, namely, $E_{i}^{\text {tot }}(\mathbf{R})$, proportional to the component $j$ of the dipole moment, divided by $p_{j}$. Of course, in our description of the electromagnetic field in the presence of scatterers, the field directly produced by the dipole $\mathbf{p}$ will result in reflection and transmission; as a consequence, the Green function will prove to be linked to the scattering operators $\mathcal{R}^{ \pm}$ and $\mathcal{T}^{ \pm}$.

It is important at this point to recall that our choice of mode decomposition of the field naturally introduces a left and a right side for a given body. Thus, we will separately discuss the cases in which the arguments $\mathbf{R}$ and $\mathbf{R}^{\prime}$ appearing in the Green function are either on the same side or on opposite sides of the body. Let us suppose first that the two points $\mathbf{R}$ and $\mathbf{R}^{\prime}$ are located on the same side $\phi$ of the body. In this case, the field directly produced by the dipole at the point $\mathbf{R}^{\prime}$ will be directly observed in $\mathbf{R}$. Moreover, this field will produce a reflected field defined in the same region $\phi$, and consequently observed in $\mathbf{R}$ as well. We argue then that for $\mathbf{R}$ and $\mathbf{R}^{\prime}$ on the same side $\phi$ of the body the Green function can be expressed as a sum of two terms, a free one independent of the scattering operators, and a reflected one proportional to $\mathcal{R}^{\phi}$. Analogously, if the first argument $\mathbf{R}$ of the Green function is located on the side $\phi$, while $\mathbf{R}^{\prime}$ is located in the $-\phi$ region, the Green function will be made up of a unique transmitted term, proportional to $\mathcal{T}^{\phi}$.

In order to make this description analytic we need the explicit expression for the dipole field propagating in direction $\phi$ given in Appendix B. As far as the free contribution is concerned (existing if $\mathbf{R}$ and $\mathbf{R}^{\prime}$ are located on the same side of the body), if $\mathbf{R}$ is on the right (left) side of $\mathbf{R}^{\prime}$, the Green function will contain the component of the field emitted by the dipole propagating toward the right (left). Let us now suppose that both $\mathbf{R}$ and $\mathbf{R}^{\prime}$ are on the same side $\phi$ of the body. In this case, apart from the direct contribution we have just discussed, the field contains a component propagating in direction $\phi$, resulting from the reflection by means of the operator $\mathcal{R}^{\phi}$ of the dipole field propagating in the opposite direction $-\phi$. Then the $i j$ component of the Green function for $\mathbf{R}$ and $\mathbf{R}^{\prime}$ on the side $\phi$ of the body reads

$$
\begin{aligned}
G_{i j}\left(\mathbf{R}, \mathbf{R}^{\prime}, \omega\right)= & G_{i j}^{(0)}\left(\mathbf{R}, \mathbf{R}^{\prime}, \omega\right)+G_{i j}^{(\mathrm{R})}\left(\mathbf{R}, \mathbf{R}^{\prime}, \omega\right), \\
G_{i j}^{(0)}\left(\mathbf{R}, \mathbf{R}^{\prime}, \omega\right)= & \frac{i \omega^{2}}{2 \epsilon_{0} c^{2}} \sum_{p} \int \frac{d^{2} \mathbf{k}}{(2 \pi)^{2}} \exp \left[i \mathbf{k} \cdot\left(\mathbf{r}-\mathbf{r}^{\prime}\right)\right] \\
& \times \frac{1}{k_{z}}\left[\theta\left(z-z^{\prime}\right)\left[\hat{\boldsymbol{\epsilon}}_{p}^{+}(\mathbf{k}, \omega)\right]_{i}\left[\hat{\boldsymbol{\epsilon}}_{p}^{+}(\mathbf{k}, \omega)\right]_{j}\right. \\
& \times \exp \left[i k_{z}\left(z-z^{\prime}\right)\right]+\theta\left(z^{\prime}-z\right)\left[\hat{\boldsymbol{\epsilon}}_{p}^{-}(\mathbf{k}, \omega)\right]_{i} \\
& \left.\times\left[\hat{\boldsymbol{\epsilon}}_{p}^{-}(\mathbf{k}, \omega)\right]_{j} \exp \left[i k_{z}\left(z^{\prime}-z\right)\right]\right], \\
G_{i j}^{(\mathrm{R})}\left(\mathbf{R}, \mathbf{R}^{\prime}, \omega\right)= & \frac{i \omega^{2}}{2 \epsilon_{0} c^{2}} \sum_{p p^{\prime}} \int \frac{d^{2} \mathbf{k}}{(2 \pi)^{2}} \int \frac{d^{2} \mathbf{k}^{\prime}}{(2 \pi)^{2}} \\
& \times \exp \left[i\left(\mathbf{k} \cdot \mathbf{r}-\mathbf{k}^{\prime} \cdot \mathbf{r}^{\prime}\right)\right] \frac{1}{k_{z}^{\prime}}\left[\hat{\boldsymbol{\epsilon}}_{p}^{\phi}(\mathbf{k}, \omega)\right]_{i}
\end{aligned}
$$

$$
\begin{aligned}
& \times\left[\hat{\boldsymbol{\epsilon}}_{p^{\prime}}^{-\phi}\left(\mathbf{k}^{\prime}, \omega\right)\right]_{j} \exp \left[i \phi\left(k_{z} z+k_{z}^{\prime} z^{\prime}\right)\right] \\
& \times\left\langle\mathbf{k}, p\left|\mathcal{R}^{\phi}\right| \mathbf{k}^{\prime}, p^{\prime}\right\rangle .
\end{aligned}
$$

On the contrary, if $\mathbf{R}\left(\mathbf{R}^{\prime}\right)$ is located on the side $\phi(-\phi)$ of the body, we observe a field, propagating in the $\phi$ direction, resulting from the transmission described by the operator $\mathcal{T}^{\phi}$ of the component of the field emitted by the dipole propagating in direction $\phi$ as well. As a consequence, in this case we conclude

$$
\begin{aligned}
G_{i j}\left(\mathbf{R}, \mathbf{R}^{\prime}, \omega\right)= & \frac{i \omega^{2}}{2 \epsilon_{0} c^{2}} \sum_{p} \int \frac{d^{2} \mathbf{k}}{(2 \pi)^{2}} \exp \left[i\left(\mathbf{k} \cdot \mathbf{r}-\mathbf{k}^{\prime} \cdot \mathbf{r}^{\prime}\right)\right] \\
& \times \sum_{p^{\prime}} \int \frac{d^{2} \mathbf{k}^{\prime}}{(2 \pi)^{2}} \frac{1}{k_{z}^{\prime}}\left[\hat{\boldsymbol{\epsilon}}_{p}^{\phi}(\mathbf{k}, \omega)\right]_{i}\left[\hat{\boldsymbol{\epsilon}}_{p^{\prime}}^{\phi}\left(\mathbf{k}^{\prime}, \omega\right)\right]_{j} \\
& \times \exp \left[i \phi\left(k_{z} z-k_{z}^{\prime} z^{\prime}\right)\right]\left\langle\mathbf{k}, p\left|\mathcal{T}^{\phi}\right| \mathbf{k}^{\prime}, p^{\prime}\right\rangle .
\end{aligned}
$$

The expressions (C1) and (C2) describe, for any position of the points $\mathbf{R}$ and $\mathbf{R}^{\prime}$ with respect to the body, the connection between the Green function and the scattering operators.

\section{APPDNDIX D: CORRELATORS OF THE FIELD EMITTED BY THE BODIES}

As discussed in Sec. VI, when a body is at thermal equilibrium with the environment at temperature $T$, the fluctuation-dissipation theorem gives complete knowledge of the correlation functions of the total field, resulting from the environmental field, the one emitted by the body, and the result of scattering processes. Nevertheless, even at thermal equilibrium the correlation functions describing the field emitted by the body are not straightforward. They were given in Eq. (45) for two components of the field propagating in the same direction, while (46) gives the corresponding quantity for counterpropagating components. The derivation of Eqs. (45) and (46) is the main scope of this appendix.

Let us consider a body at thermal equilibrium at temperature $T$ and described by the scattering operators $\mathcal{R}^{ \pm}$and $\mathcal{T}^{ \pm}$. The first step of our calculation is to write the expression for the total field both on the left and on the right sides of the body. Their components at frequency $\omega$ are given by

$$
\begin{aligned}
\mathbf{E}^{(\text {tot }) \phi}(\mathbf{R}, \omega)= & \mathbf{E}^{(\mathrm{env})-\phi}(\mathbf{R}, \omega)+\mathbf{E}^{(\mathrm{b}) \phi}(\mathbf{R}, \omega) \\
& +\mathbf{E}^{(\mathrm{re}) \phi}(\mathbf{R}, \omega)+\mathbf{E}^{(\mathrm{tr}) \phi}(\mathbf{R}, \omega) .
\end{aligned}
$$

In this expression the superscript $\phi$ for the total field in the left-hand side (LHS) refers to the region we are considering, while for the four fields in the RHS it corresponds, as usual in the rest of the paper, to the direction of propagation. Equation (D1) tells us that the total field in region $\phi$ contains first the environmental field propagating in direction $-\phi$ (i.e., toward the body) and of course the field $\mathbf{E}^{(\mathrm{b}) \phi}$ emitted by the body itself and propagating in direction $\phi$. Moreover, the environmental field $\mathbf{E}^{(\mathrm{env})-\phi}$ produces a reflected field $\mathbf{E}^{(\mathrm{re}) \phi}$ connected to $\mathbf{E}^{(\mathrm{env})-\phi}$ by the operator $\mathcal{R}^{\phi}$. Finally, in region $\phi$ there is a field $\mathbf{E}^{(\mathrm{tr}) \phi}$ resulting from the transmission of the environmental field $\mathbf{E}^{(\text {env) } \phi}$ (existing in the other region $-\phi$ ) by the operator $\mathcal{T}^{\phi}$. 
We are looking for correlation functions such as

$$
\left\langle E_{p}^{(\mathrm{b}) \phi}(\mathbf{k}, \omega) E_{p^{\prime}}^{(\mathbf{b}) \phi^{\prime \dagger}}\left(\mathbf{k}^{\prime}, \omega^{\prime}\right)\right\rangle_{\mathrm{sym}}
$$

of the field emitted by the body, both for $\phi=\phi^{\prime}$ and for $\phi \neq \phi^{\prime}$. Let us start with the case of copropagating components of the field, namely, with the case $\phi=\phi^{\prime}$. It is first useful to calculate the correlation function of the $i$ and $j$ components of Eq. (D1), for two couples of coordinates $(\mathbf{R}, \omega)$ and $\left(\mathbf{R}^{\prime}, \omega^{\prime}\right)$. As for the LHS, the result is directly given by the fluctuation-dissipation theorem stated in Eq. (39). The discussion of the RHS requires a more accurate analysis. We first point out that we already know the correlation function characterizing the environmental field: it is given by Eq. (43) and it also tells us that two counterpropagating components of this field are uncorrelated. As a consequence, $\mathbf{E}^{(\text {env) }-\phi}$ is correlated (apart from with itself) with the reflected field $\mathbf{E}^{(\mathrm{re}) \phi}$, but not with the transmitted field $\mathbf{E}^{(\mathrm{tr}) \phi}$, coming from the counterpropagating component of the environmental field. As a consequence $\mathbf{E}^{(\mathrm{tr}) \phi}$ is correlated only with itself; the same holds for the field $\mathbf{E}^{(\mathrm{b}) \phi}$, since the field produced by the body alone is of course uncorrelated with everything that results from the environment. We now observe that, since the reflected and transmitted fields are connected by means of scattering operators to the environmental field, whose correlation functions are known, the only unknown remaining is the correlation function

$$
\begin{gathered}
\left\langle E_{i}^{(\mathrm{b}) \phi}(\mathbf{R}, \omega) E_{j}^{(\mathrm{b}) \phi \dagger}\left(\mathbf{R}^{\prime}, \omega^{\prime}\right)\right\rangle_{\mathrm{sym}}, \\
\left\langle E_{i}^{(\mathrm{env})-\phi}(\mathbf{R}, \omega) E_{j}^{(\mathrm{env})-\phi^{\dagger}}\left(\mathbf{R}^{\prime}, \omega^{\prime}\right)\right\rangle=\sum_{p p^{\prime}} \exp \left[-i \phi\left(k_{z} z-k_{z}^{\prime *} z^{\prime}\right)\right]\left[\hat{\boldsymbol{\epsilon}}_{p}^{-\phi}(\mathbf{k}, \omega)\right]_{i}\left[\hat{\boldsymbol{\epsilon}}_{p^{\prime}}^{-\phi}\left(\mathbf{k}^{\prime}, \omega\right)\right]_{j}^{*}\left\langle\mathbf{k}, p\left|C^{(3)}\right| \mathbf{k}^{\prime}, p^{\prime}\right\rangle, \\
\left\langle E_{i}^{(\mathrm{re}) \phi}(\mathbf{R}, \omega) E_{j}^{(\mathrm{re}) \phi \dagger}\left(\mathbf{R}^{\prime}, \omega^{\prime}\right)\right\rangle=\sum_{p p^{\prime}} \exp \left[i \phi\left(k_{z} z-k_{z}^{\prime *} z^{\prime}\right)\right]\left[\hat{\boldsymbol{\epsilon}}_{p}^{\phi}(\mathbf{k}, \omega)\right]_{i}\left[\hat{\boldsymbol{\epsilon}}_{p^{\prime}}^{\phi}\left(\mathbf{k}^{\prime}, \omega\right)\right]_{j}^{*}\left\langle\mathbf{k}, p\left|\mathcal{R}^{\phi} C^{(3)} \mathcal{R}^{\phi \dagger}\right| \mathbf{k}^{\prime}, p^{\prime}\right\rangle, \\
\left\langle E_{i}^{(\mathrm{env})-\phi}(\mathbf{R}, \omega) E_{j}^{(\mathrm{re}) \phi \dagger}\left(\mathbf{R}^{\prime}, \omega^{\prime}\right)\right\rangle=\sum_{p p^{\prime}} \exp \left[-i \phi\left(k_{z} z+k_{z}^{\prime} z^{\prime}\right)\right]\left[\hat{\boldsymbol{\epsilon}}_{p}^{-\phi}(\mathbf{k}, \omega)\right]_{i}\left[\hat{\boldsymbol{\epsilon}}_{p^{\prime}}^{\phi}\left(\mathbf{k}^{\prime}, \omega\right)\right]_{j}^{*}\left\langle\mathbf{k}, p\left|C^{(3)} \mathcal{R}^{\phi \dagger}\right| \mathbf{k}^{\prime}, p^{\prime}\right\rangle, \\
\left\langle E_{i}^{(\mathrm{re}) \phi}(\mathbf{R}, \omega) E_{j}^{(\mathrm{env})-\phi^{\dagger}}\left(\mathbf{R}^{\prime}, \omega^{\prime}\right)\right\rangle=\sum_{p p^{\prime}} \exp \left[i \phi\left(k_{z} z+k_{z}^{*} z^{\prime}\right)\right]\left[\hat{\boldsymbol{\epsilon}}_{p}^{\phi}(\mathbf{k}, \omega)\right]_{i}\left[\hat{\boldsymbol{\epsilon}}_{p^{\prime}}^{-\phi}\left(\mathbf{k}^{\prime}, \omega\right)\right]_{j}^{*}\left\langle\mathbf{k}, p\left|\mathcal{R}^{\phi} C^{(3)}\right| \mathbf{k}^{\prime}, p^{\prime}\right\rangle, \\
\left\langle E_{i}^{\text {(tr) } \phi}(\mathbf{R}, \omega) E_{j}^{(\mathrm{tr}) \phi \dagger}\left(\mathbf{R}^{\prime}, \omega^{\prime}\right)\right\rangle=\sum_{p p^{\prime}} \exp \left[i \phi\left(k_{z} z-k_{z}^{\prime *} z^{\prime}\right)\right]\left[\hat{\boldsymbol{\epsilon}}_{p}^{\phi}(\mathbf{k}, \omega)\right]_{i}\left[\hat{\boldsymbol{\epsilon}}_{p^{\prime}}^{\phi}\left(\mathbf{k}^{\prime}, \omega\right)\right]_{j}^{*}\left\langle\mathbf{k}, p\left|\mathcal{T}^{\phi} C^{(3)} \mathcal{T}^{\phi \dagger}\right| \mathbf{k}^{\prime}, p^{\prime}\right\rangle, \\
\left\langle E_{i}^{(\mathrm{b}) \phi}(\mathbf{R}, \omega) E_{j}^{(\mathrm{b}) \phi \dagger}\left(\mathbf{R}^{\prime}, \omega^{\prime}\right)\right\rangle=\sum_{p p^{\prime}} \exp \left[i \phi\left(k_{z} z-k_{z}^{*} z^{\prime}\right)\right]\left[\hat{\boldsymbol{\epsilon}}_{p}^{\phi}(\mathbf{k}, \omega)\right]_{i}\left[\hat{\boldsymbol{\epsilon}}_{p^{\prime}}^{\phi}\left(\mathbf{k}^{\prime}, \omega\right)\right]_{j}^{*}\left\langle\mathbf{k}, p\left|C^{\phi \phi}\right| \mathbf{k}^{\prime}, p^{\prime}\right\rangle .
\end{gathered}
$$

We now need to calculate the LHS by using the fluctuationdissipation theorem (39) and inserting the explicit expression found in Appendix $\mathrm{C}$ of the Green function as a function of the scattering operators. Since in this case we are calculating the correlator of $E_{i}^{(\mathrm{tot}) \phi}$ with $E_{j}^{(\mathrm{tot}) \phi}$, we are using the Green function calculated at positions $\mathbf{R}$ and $\mathbf{R}^{\prime}$, which are on the same side $\phi$ of the body. We will thus make use of Eq. (C1) to connect the Green function to the scattering operators. In particular, the fluctuation-dissipation theorem tells us that we need to calculate the imaginary part of the Green function (C1). Starting from the free term $G_{i j}^{(0)}$ and inserting a term
$(2 \pi)^{2} \delta\left(\mathbf{k}-\mathbf{k}^{\prime}\right)$ integrated over $\mathbf{k}^{\prime}$ as well as a $\delta_{p p^{\prime}}$ in order to have the desired factor $\exp \left[i\left(\mathbf{k} \cdot \mathbf{r}-\mathbf{k}^{\prime} \cdot \mathbf{r}^{\prime}\right)\right]$ and the same structure as the terms in (D5) [we remark here that the factor $2 \pi \delta\left(\omega-\omega^{\prime}\right)$ is already in the fluctuation-dissipation theorem (39)], we obtain

$$
\begin{aligned}
\operatorname{Im}_{i j}^{(0)}\left(\mathbf{R}, \mathbf{R}^{\prime}, \omega\right) \\
=\frac{\omega^{2}}{4 \epsilon_{0} c^{2}} \sum_{p p^{\prime}} \delta_{p p^{\prime}}(2 \pi)^{2} \delta\left(\mathbf{k}-\mathbf{k}^{\prime}\right)\left\{\theta ( z - z ^ { \prime } ) \left[\frac{1}{k_{z}}\right.\right. \\
\quad \times\left[\hat{\boldsymbol{\epsilon}}_{p}^{+}(\mathbf{k}, \omega)\right]_{i}\left[\hat{\boldsymbol{\epsilon}}_{p^{\prime}}^{+}\left(\mathbf{k}^{\prime}, \omega\right)\right]_{j} \exp \left[i\left(k_{z} z-k_{z}^{\prime} z^{\prime}\right)\right]
\end{aligned}
$$




$$
\begin{aligned}
& +\frac{1}{k_{z}^{*}}\left[\hat{\boldsymbol{\epsilon}}_{p}^{+}(-\mathbf{k}, \omega)\right]_{i}^{*}\left[\hat{\boldsymbol{\epsilon}}_{p^{\prime}}^{+}\left(-\mathbf{k}^{\prime}, \omega\right)\right]_{j}^{*} \\
& \left.\times \exp \left[-i\left(k_{z}^{*} z-k_{z}^{*} z^{\prime}\right)\right]\right] \\
& +\theta\left(z^{\prime}-z\right)\left[\frac{1}{k_{z}}\left[\hat{\boldsymbol{\epsilon}}_{p}^{-}(\mathbf{k}, \omega)\right]_{i}\left[\hat{\boldsymbol{\epsilon}}_{p^{\prime}}^{-}\left(\mathbf{k}^{\prime}, \omega\right)\right]_{j}\right. \\
& \times \exp \left[i\left(k_{z}^{\prime} z^{\prime}-k_{z} z\right)\right] \\
& +\frac{1}{k_{z}^{*}}\left[\hat{\boldsymbol{\epsilon}}_{p}^{-}(-\mathbf{k}, \omega)\right]_{i}^{*}\left[\hat{\boldsymbol{\epsilon}}_{p^{\prime}}^{-}\left(-\mathbf{k}^{\prime}, \omega\right)\right]_{j}^{*} \\
& \left.\left.\times \exp \left[-i\left(k_{z}^{*} z^{\prime}-k_{z}^{*} z\right)\right]\right]\right\},
\end{aligned}
$$

where for the terms obtained by complex conjugation we made a change of variable from $\mathbf{k}$ to $\mathbf{- k}$. Making use of the properties of the polarization unit vectors, it is easy to show that each term multiplying a step function $\theta$ is zero in the evanescent sector of $\mathbf{k}$ (and then $\mathbf{k}^{\prime}$ ). After simple algebraic manipulations the imaginary part of $G_{i j}^{(0)}$ finally takes the form

$$
\begin{aligned}
\operatorname{Im}_{i j} & (0) \\
= & \frac{\omega^{2}}{4 \epsilon_{0} c^{2}} \sum_{p p^{\prime}} \delta_{p p^{\prime}}(2 \pi)^{2} \delta\left(\mathbf{k}-\mathbf{k}^{\prime}\right) \theta(\omega-c k) \frac{1}{k_{z}} \\
& \times\left[\left[\hat{\boldsymbol{\epsilon}}_{p}^{+}(\mathbf{k}, \omega)\right]_{i}\left[\hat{\boldsymbol{\epsilon}}_{p^{\prime}}^{+}\left(\mathbf{k}^{\prime}, \omega\right)\right]_{j} \exp \left[i\left(k_{z} z-k_{z}^{\prime} z^{\prime}\right)\right]\right. \\
& \left.+\left[\hat{\boldsymbol{\epsilon}}_{p}^{-}(\mathbf{k}, \omega)\right]_{i}\left[\hat{\boldsymbol{\epsilon}}_{p^{\prime}}^{-}\left(\mathbf{k}^{\prime}, \omega\right)\right]_{j} \exp \left[i\left(k_{z}^{\prime} z^{\prime}-k_{z} z\right)\right]\right] .
\end{aligned}
$$

Observing from Eq. (43) that

$$
\begin{aligned}
\left\langle\mathbf{k}, p\left|C^{(3)}\right| \mathbf{k}^{\prime}, p^{\prime}\right\rangle= & \frac{\omega}{2 \epsilon_{0} c^{2}} N(\omega, T) \delta_{p p^{\prime}}(2 \pi)^{2} \delta\left(\mathbf{k}-\mathbf{k}^{\prime}\right) \\
& \times \theta(\omega-c k) \frac{1}{k_{z}}
\end{aligned}
$$

and taking back the factor $\frac{2}{\omega} N(\omega, T)$ in Eq. (39), we conclude that the term (D7) cancels the first term in (D5) and gives in the LHS a contribution

$$
\begin{aligned}
& \sum_{p p^{\prime}} \exp \left[i \phi\left(k_{z} z-k_{z}^{\prime}{ }^{*} z^{\prime}\right)\right]\left[\hat{\boldsymbol{\epsilon}}_{p}^{\phi}(\mathbf{k}, \omega)\right]_{i}\left[\hat{\boldsymbol{\epsilon}}_{p^{\prime}}^{\phi}\left(\mathbf{k}^{\prime}, \omega\right)\right]_{j} \\
& \quad \times\left\langle\mathbf{k}, p\left|C^{(3)}\right| \mathbf{k}^{\prime}, p^{\prime}\right\rangle .
\end{aligned}
$$

We are now left with the calculation of the imaginary part of $G_{i j}^{(\mathrm{R})}$. We have

$$
\begin{aligned}
& \operatorname{Im}_{i j}^{(\mathrm{R})}\left(\mathbf{R}, \mathbf{R}^{\prime}, \omega\right) \\
& =\frac{\omega^{2}}{4 \epsilon_{0} c^{2}} \sum_{p p^{\prime}}\left[\frac{1}{k_{z}^{\prime}}\left[\hat{\boldsymbol{\epsilon}}_{p}^{\phi}(\mathbf{k}, \omega)\right]_{i}\left[\hat{\boldsymbol{\epsilon}}_{p^{\prime}}^{-\phi}\left(\mathbf{k}^{\prime}, \omega\right)\right]_{j}\right.
\end{aligned}
$$

$$
\begin{aligned}
& \times \exp \left[i \phi\left(k_{z} z+k_{z}^{\prime} z^{\prime}\right)\right]\left\langle\mathbf{k}, p\left|\mathcal{R}^{\phi}\right| \mathbf{k}^{\prime}, p^{\prime}\right\rangle \\
& +\frac{1}{k_{z}^{\prime *}}\left[\hat{\boldsymbol{\epsilon}}_{p}^{-\phi}(\mathbf{k}, \omega)\right]_{i}^{*}\left[\hat{\boldsymbol{\epsilon}}_{p^{\prime}}^{\phi}\left(\mathbf{k}^{\prime}, \omega\right)\right]_{j}^{*} \exp \left[-i \phi\left(k_{z}^{*} z+k_{z}^{\prime *} z^{\prime}\right)\right] \\
& \left.\times(-1)^{p+p^{\prime}}\left\langle-\mathbf{k}^{\prime}, p^{\prime}\left|\mathcal{R}^{\phi \dagger}\right|-\mathbf{k}, p\right\rangle\right],
\end{aligned}
$$

where the second term was obtained by the change of variables $\left(\mathbf{k}, \mathbf{k}^{\prime}\right) \longrightarrow\left(-\mathbf{k},-\mathbf{k}^{\prime}\right)$ and using the properties of the polarization unit vectors (8). Starting from the first term we note that its part which is propagative in $\mathbf{k}^{\prime}$ exactly cancels the fourth term in Eq. (D5). Observing that in the evanescent sector we have $k_{z}^{*}=-k_{z}^{\prime}$ and using again (8), we are left from the first term with a contribution

$$
\begin{aligned}
& \frac{\omega^{2}}{4 \epsilon_{0} c^{2}} \sum_{p p^{\prime}}\left[\hat{\boldsymbol{\epsilon}}_{p}^{\phi}(\mathbf{k}, \omega)\right]_{i}\left[\hat{\boldsymbol{\epsilon}}_{p^{\prime}}^{\phi}\left(\mathbf{k}^{\prime}, \omega\right)\right]_{j}^{*} \\
& \quad \times \exp \left[i \phi\left(k_{z} z-k_{z}^{\prime *} z^{\prime}\right)\right]\left\langle\mathbf{k}, p\left|\mathcal{R}^{\phi} \mathcal{P}_{-1}^{(\mathrm{ew})}\right| \mathbf{k}^{\prime}, p^{\prime}\right\rangle .
\end{aligned}
$$

As far as the second term is concerned, we exploit the reciprocity relations of scattering operators presented in Appendix B to conclude that

$$
\frac{1}{k_{z}^{\prime *}}(-1)^{p+p^{\prime}}\left\langle-\mathbf{k}^{\prime}, p^{\prime}\left|\mathcal{R}^{\phi \dagger}\right|-\mathbf{k}, p\right\rangle=\frac{1}{k_{z}^{*}}\left\langle\mathbf{k}, p\left|\mathcal{R}^{\phi \dagger}\right| \mathbf{k}^{\prime}, p^{\prime}\right\rangle \text {. }
$$

As a consequence the propagative part with respect to $\mathbf{k}$ of this second term analogously cancels the third term in (D5), and we are left with

$$
\begin{aligned}
& -\frac{\omega^{2}}{4 \epsilon_{0} c^{2}} \sum_{p p^{\prime}}\left[\hat{\boldsymbol{\epsilon}}_{p}^{\phi}(\mathbf{k}, \omega)\right]_{i}\left[\hat{\boldsymbol{\epsilon}}_{p^{\prime}}^{\phi}\left(\mathbf{k}^{\prime}, \omega\right)\right]_{j}^{*} \\
& \quad \times \exp \left[i \phi\left(k_{z} z-k_{z}^{*} z^{\prime}\right)\right]\left\langle\mathbf{k}, p\left|\mathcal{P}_{-1}^{(\mathrm{ew})} \mathcal{R}^{\phi^{\dagger}}\right| \mathbf{k}^{\prime}, p^{\prime}\right\rangle .
\end{aligned}
$$

Having considered all the terms in the equality (D4), we remain now with quantities which all have exactly the same structure, namely, the same sum over $p$ and $p^{\prime}$, the same $z$-dependent exponential, and the same polarization unit vectors. As a consequence we can identify the matrices whose elements are calculated between $(\mathbf{k}, p)$ and $\left(\mathbf{k}^{\prime}, p^{\prime}\right)$ and obtain immediately the equality (45).

In the case of the correlation function between two components of the field emitted by the body propagating in two opposite directions, the structure of the calculation is the same. Now one has to use Eq. (C2) instead of (C1) in order to connect the Green function to the scattering operators. The analytic expression (46) for the correlation function for counterpropagating fields has already been given in the paper.
[1] D. Polder and M. Van Hove, Phys. Rev. B 4, 3303 (1971).

[2] H. B. G. Casimir, Proc. K. Ned. Akad. Wet. 51, 793 (1948).

[3] H. B. G. Casimir and D. Polder, Phys. Rev. 73, 360 (1948).

[4] I. E. Dzyaloshinskii, E. M. Lifshitz, and L. P. Pitaevskii, Adv. Phys. 10, 165 (1961).
[5] S. M. Rytov, Y. A. Kravtsov, and V. I. Tatarskii, Principles of Statistical Radiophysics, Vol. 3: Elements of Random Fields (Springer, Berlin, 1989).

[6] K. Joulain, J.-P. Mulet, F. Marquier, R. Carminati, and J.-J. Greffet, Surf. Sci. Rep. 57, 59 (2005). 
[7] Casimir Physics, Lecture Notes in Physics Vol. 834, edited by D. Dalvit, P. Milonni, D. Roberts, and F. Da Rosa (SpringerVerlag, Berlin, 2011).

[8] A. O. Sushkov, W. J. Kim, D. A. R. Dalvit, and S. K. Lamoreaux, Nature Phys. 7, 230 (2011).

[9] M. Antezza, L. P. Pitaevskii, and S. Stringari, Phys. Rev. Lett. 95, 113202 (2005).

[10] M. Antezza, L. P. Pitaevskii, S. Stringari, and V. B. Svetovoy, Phys. Rev. Lett. 97, 223203 (2006).

[11] M. Antezza, J. Phys. A 39, 6117 (2006).

[12] J. M. Obrecht, R. J. Wild, M. Antezza, L. P. Pitaevskii, S. Stringari, and E. A. Cornell, Phys. Rev. Lett. 98, 063201 (2007).

[13] M. Antezza, L. P. Pitaevskii, S. Stringari, and V. B. Svetovoy, Phys. Rev. A 77, 022901 (2008).

[14] S. Y. Buhmann and S. Scheel, Phys. Rev. Lett. 100, 253201 (2008).

[15] Y. Sherkunov, Phys. Rev. A 79, 032101 (2009).

[16] G. Bimonte, Phys. Rev. A 80, 042102 (2009).

[17] R. O. Behunin and B.-L. Hu, Phys. Rev. A 82, 022507 (2010).

[18] R. Behunin and B.-L. Hu, J. Phys. A 43, 012001 (2010).

[19] R. Behunin and B.-L. Hu, Phys. Rev. A 84, 012902 (2011).

[20] A. I. Volokitin and B. N. J. Persson, Rev. Mod. Phys. 79, 1291 (2007).

[21] E. Rousseau, A. Siria, G. Jourdan, S. Volz, F. Comin, J. Chevrier, and J.-J. Greffet, Nature Photon. 3, 514 (2009).

[22] S. Shen, A. Narayanaswamy, and G. Chen, Nano Lett. 9, 2909 (2009).

[23] T. Kralik, P. Hanzelka, V. Musilova, A. Srnka, and M. Zobac, Rev. Sci. Instrum. 82, 055106 (2011).

[24] R. S. Ottens, V. Quetschke, S. Wise, A. A. Alemi, R. Lundock, G. Mueller, D. H. Reitze, D. B. Tanner, and B. F. Whiting, Phys. Rev. Lett. 107, 014301 (2011).

[25] P. Ben-Abdallah, K. Joulain, J. Drevillon, and G. Domingues, J. Appl. Phys. 106, 044036 (2009).

[26] P. Ben-Abdallah and K. Joulain, Phys. Rev. B 82, 121419 (2010).

[27] S.-A. Biehs, P. Ben-Abdallah, F. S. S. Rosa, K. Joulain, and J.-J. Greffet, e-print arXiv:1103.2361.
[28] R. Messina and M. Antezza, Europhys. Lett. 95, 61002 (2011).

[29] M. Krüger, T. Emig, and M. Kardar, Phys. Rev. Lett. 106, 210404 (2011).

[30] M. Krüger, T. Emig, G. Bimonte, and M. Kardar, Europhys. Lett. 95, 21002 (2011).

[31] G. Bimonte, T. Emig, M. Krüger, and M. Kardar, e-print arXiv:1107.1597.

[32] A. W. Rodriguez, O. Ilic, P. Bermel, I. Celanovic, J. D. Joannopoulos, M. Soljačić, and S. G. Johnson, Phys. Rev. Lett. 107, 114302 (2011).

[33] A. P. McCauley, M. T. Homer Reid, M. Krüger, and S. G. Johnson, e-print arXiv:1107.2111, Phys. Rev. Lett. (to be published).

[34] A. Lambrecht, P. A. Maia Neto, and S. Reynaud, New J. Phys. 8, 243 (2006).

[35] S. J. Rahi, T. Emig, N. Graham, R. L. Jaffe, and M. Kardar, Phys. Rev. D 80, 085021 (2009).

[36] L. D. Landau and E. M. Lifshitz, Electrodynamics of Continuous Media (Pergamon Press, Oxford, 1963).

[37] C. Henkel, K. Joulain, J.-P. Mulet, and J.-J. Greffet, J. Opt. A: Pure Appl. Opt. 4, S109 (2002).

[38] R. Messina, D. A. R. Dalvit, P. A. Maia Neto, A. Lambrecht, and S. Reynaud, Phys. Rev. A 80, 022119 (2009).

[39] Handbook of Optical Constants of Solids, edited by E. Palik (Academic Press, New York, 1998).

[40] S. Dimopoulos and A. A. Geraci, Phys. Rev. D 68, 124021 (2003).

[41] I. Carusotto, L. Pitaevskii, S. Stringari, G. Modugno, and M. Inguscio, Phys. Rev. Lett. 95, 093202 (2005).

[42] P. Wolf, P. Lemonde, A. Lambrecht, S. Bize, A. Landragin, and A. Clairon, Phys. Rev. A 75, 063608 (2007).

[43] F. Sorrentino, A. Alberti, G. Ferrari, V. V. Ivanov, N. Poli, M. Schioppo, and G. M. Tino, Phys. Rev. A 79, 013409 (2009).

[44] R. Messina, S. Pelisson, M.-C. Angonin, and P. Wolf, Phys. Rev. A 83, 052111 (2011).

[45] R. Carminati, M. Nieto-Vesperinas, and J.-J. Greffet, J. Opt. Soc. Am. A 15, 706 (1998).

[46] M. Nieto-Vesperinas, Scattering and Diffraction in Physical Optics (Wiley, New York, 1991). 